\title{
MULTIMODAL SPECTROSCOPY OF SINGLE FLUORESCENT NACOPROBES : PHOTOPHYSICS AND CHARACTERIZATION
}

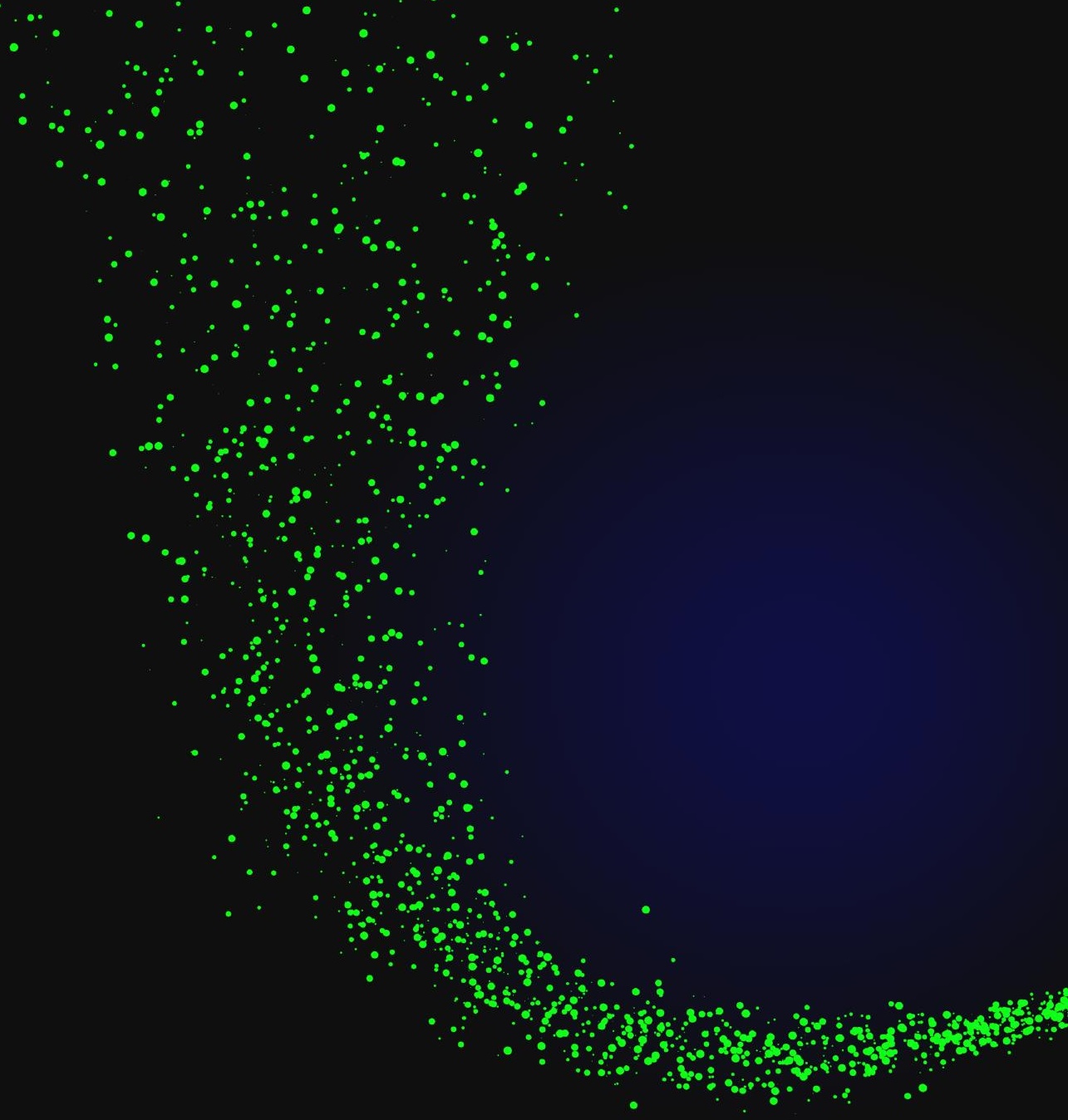

Martijn Stopel 


\section{MULTIMODAL SPECTROSCOPY OF SINGLE FLUORESCENT NANOPROBES: PHOTOPHYSICS AND CHARACTERIZATION}

Martijn H.W. Stopel 
Members of the dissertation committee:

Prof. dr. V. Subramaniam University of Twente (promotor)

Dr. C. Blum University of Twente (assistent promotor)

Prof. dr. A.M. Brouwer University of Amsterdam

Prof. dr. M.A.G.J. Orrit Leiden University

Prof. dr. G.J. Vancso University of Twente

Prof. dr. A.P. Mosk University of Twente

Prof. dr. ir. J.F. Dijkssman University of Twente (chairman and secretary)

This work was funded by

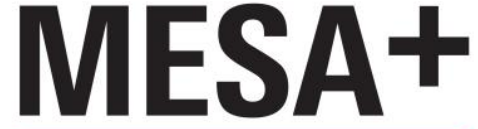

INSTITUTE FOR NANOTECHNOLOGY

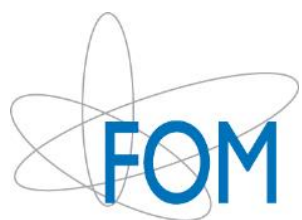

MESA +

Institute for Nanotechnology

University of Twente, PO Box 217,

NL-7500 AE Enschede

Foundation for

Fundamental Research on Matter

PO Box 3021, 3502 GA Utrecht

Copyright (C) 2014 by Martijn H.W. Stopel

All rights reserved. No part of this book may be reproduced or transmitted, in any form or by any means, electronic or mechanical, including photocopying, microfilming, and recording, or by any information storage or retrieval system, without prior written permission of the author.

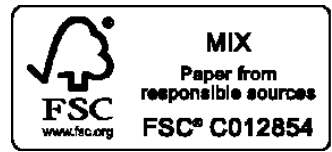

This thesis was printed by Gildeprint Drukkerijen, Enschede, The Netherlands ISBN: 978-90-365-3753-7

DOI: $\quad 10.3990 / 1.9789036537537$ 


\title{
MULTIMODAL SPECTROSCOPY OF SINGLE FLUORESCENT NANOPROBES: PHOTOPHYSICS AND CHARACTERIZATION
}

\author{
PROEFSCHRIFT
}

\author{
ter verkrijging van \\ de graad van doctor aan de Universiteit Twente, \\ op gezag van de rector magnificus, \\ prof. dr. H. Brinksma, \\ volgens besluit van het College voor Promoties \\ in het openbaar te verdedigen \\ op donderdag 25 september 2014 om 16.45 uur
}

door

Martijn Hendrikus Wilhelmus Stopel

geboren op 23 juli 1984

te Hengelo (OV) 
Dit proefschrift is goedgekeurd door:

Prof. dr. V. Subramaniam University of Twente (promotor)

Dr. C. Blum University of Twente (assistent promotor) 


\section{CONTENTS}

CHAPTER 1: INTRODUCTION 1

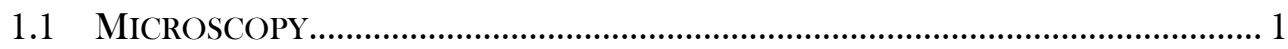

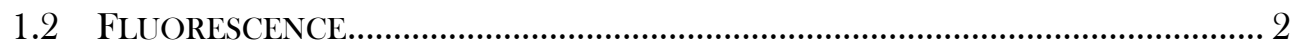

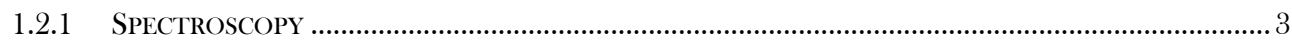

1.2.2 TIME-RESOLVED CHARACTERISATION .................................................................................... 4

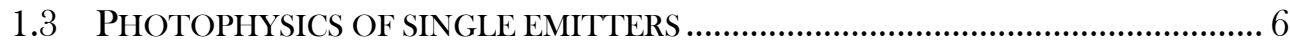

1.4 FLUORESCENT PROBES USED IN THE THESIS ........................................... 7

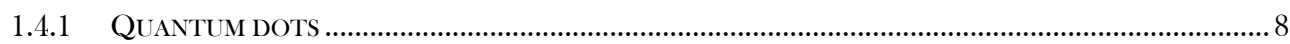

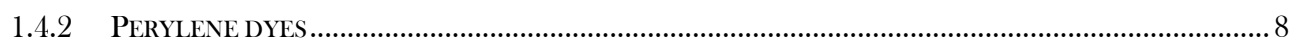

1.4.3 DSRED (RED FLUORESCENT PROTEIN) ………………………………………………..........

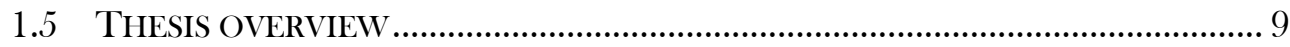

CHAPTER 2: MULTIMODAL FLUORESCENCE IMAGING

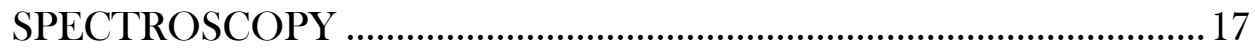

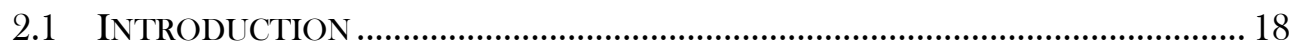

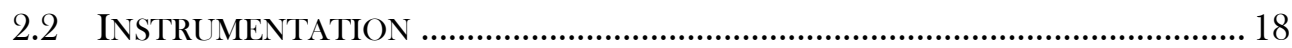

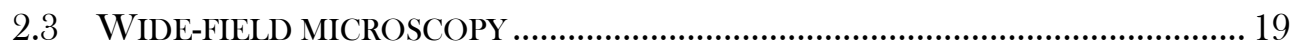

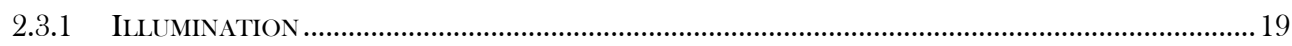

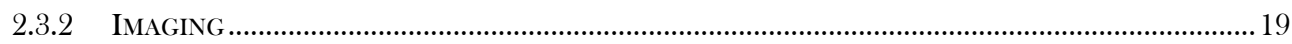

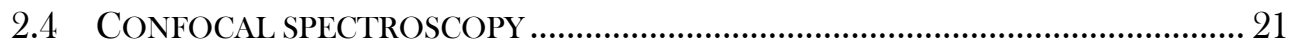

2.4.1 EXCITATION SOURCE .............................................................................................................21

2.4.2 CONFOCAL DETECTORS …………………………………………………………………...... 22

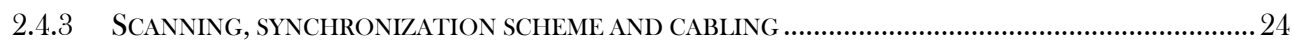

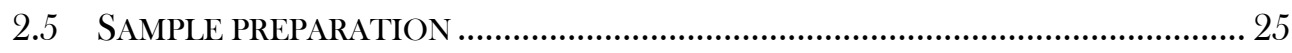

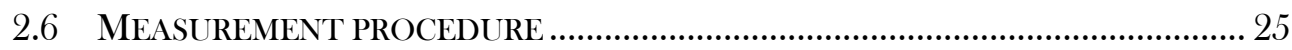

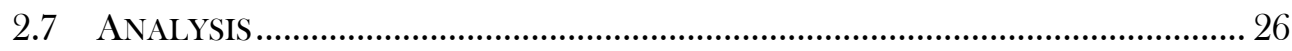

2.7.1 INTENSITY AND LIFETIME ………………………………………………………………...... 27

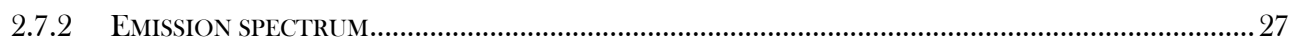

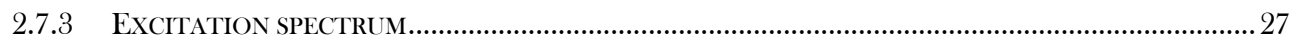

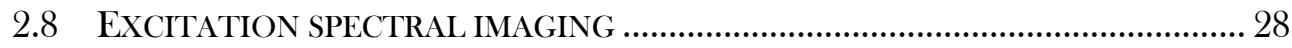

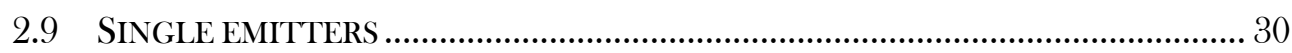




\section{CHAPTER 3: ROOM TEMPERATURE EXCITATION SPECTROSCOPY OF SINGLE QUANTUM DOTS}

3.1 PROBING SPECTRAL ABSORBANCE OF SINGLE EMITTERS .............................. 36

3.2 SETUP AND MEASUREMENT PROCEDURE …….............................................. 37

3.2.1 SWEEPING EXCITATION SOURCE ……………………………………………………………..... 37

3.2.2 SINGLE EMITTER SENSITIVE DETECTION ................................................................................ 38

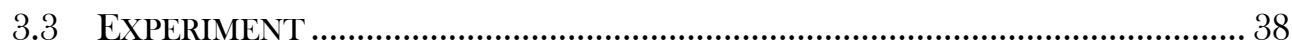

3.3.1 RECORDING SINGLE EMITTER EXCITATION SPECTRA ………………………………………...... 38

3.3.2 EXCITATION SPECTRA OF SINGLE QUANTUM DOTS ………………………………………….... 40

3.3.3 INDIVIDUAL SPECTRAL PROPERTIES OF THE SINGLE EMITTERS ................................................... 42

3.3.4 DARK STATE DEPENDENCE ON EXCITATION WAVELENGTH? ………………………………….... 45

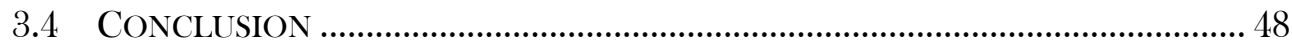

CHAPTER 4: EXCITATION SPECTRA AND STOKES SHIFT MEASUREMENTS OF SINGLE ORGANIC DYES AT ROOM-

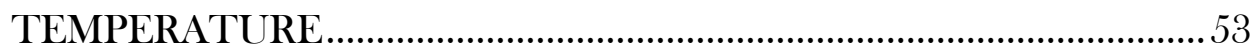

4.1 EXCITATION SPECTRA OF SINGLE ORGANIC FLUOROPHORES......................... 54

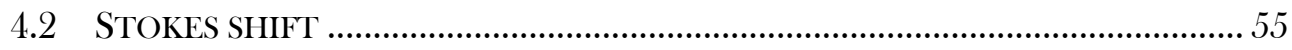

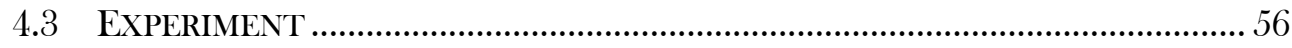

4.4 EXCITATION AND EMISSION SPECTRA OF SINGLE ORGANIC EMITTERS ........59

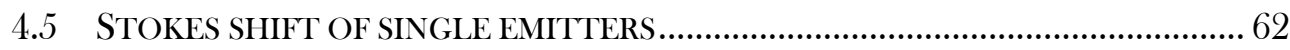

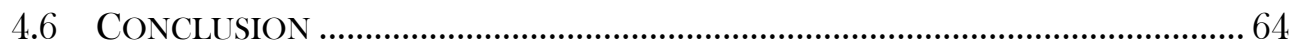

\section{CHAPTER 5: BLINKING STATISTICS OF COLLOIDAL QUANTUM DOTS AT DIFFERENT EXCITATION WAVELENGTHS ...............69}

5.1 BLINKING IN MODERN FLUORESCENCE MICROSCOPY ……............................ 70

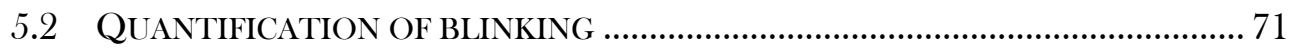

5.3 EVALUATION OF BLINKING ANALYSIS METHODS ……………........................ 73

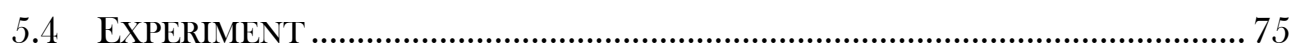

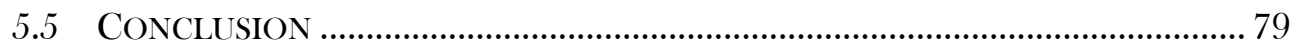

ii 
CHAPTER 6: DISCRETE DISTRIBUTION OF GREY STATES IN SINGLE CDSE/ZNS CORE-SHELL QUANTUM DOTS

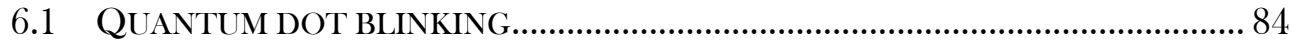

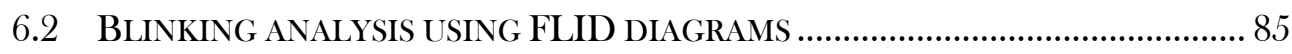

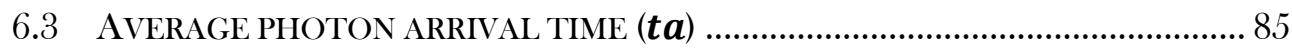

6.4 OBSERVATION OF DEFINITE STATES IN SINGLE QUANTUM DOT EMISSION

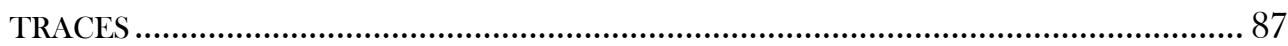

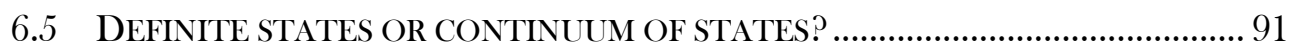

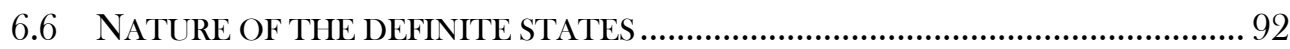

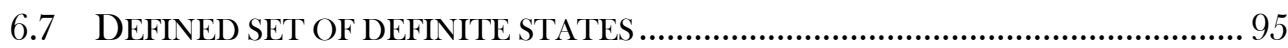

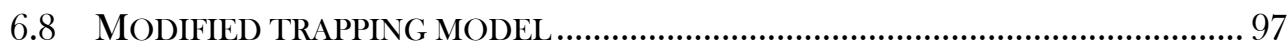

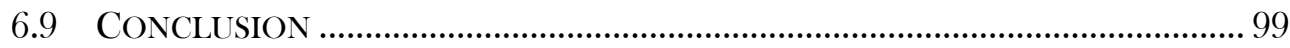

CHAPTER 7: FUTURE APPLICATIONS OF MULTIMODAL FLIM103

7.1 MEASUREMENT OF THE QUANTUM EFFICIENCY OF FLUOROPHORES ........ 104

7.1.1 CONVENTIONAL METHOD ……………………………………………………………….... 104

7.1.2 FLUORESCENCE LIFETIME BASED METHOD ……………………………………………….... 104

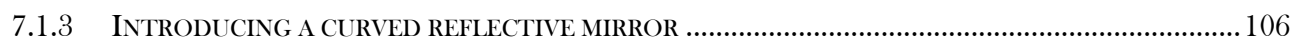

7.2 SURFACE PATTERNING OF FLUOROPHORES ………................................... 110

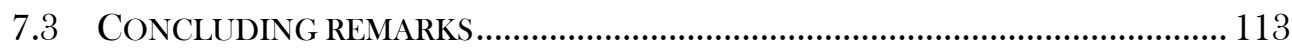

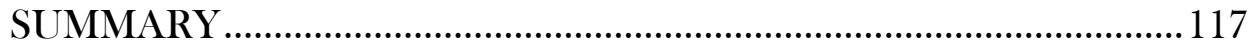

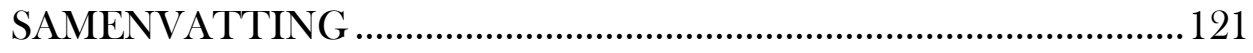

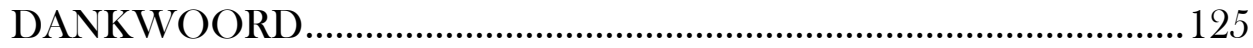

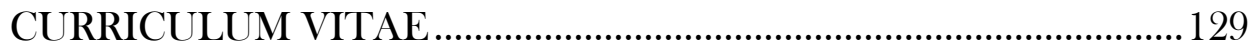

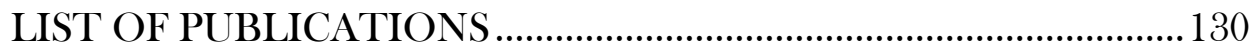





\section{Chapter 1}

\section{Introduction}

\subsection{Microscopy}

Bright field microscopy is an optical microscopy technique that was first discovered by the two Dutch spectacle makers Zaccharias and Hans Janssen around 1590. While experimenting with two lenses inside a tube, they discovered that nearby objects could be magnified. Galileo improved the microscope in 1609 by adding a focusing device and worked out the basic principles of lenses. Later on in the $17^{\text {th }}$ century, Antoni van Leeuwenhoek managed to build microscopes that were capable of magnifying up to $480 \mathrm{X}$, which led to the discovery of a range of micro-organisms like bacteria, yeast, enabling the visualization of the vivid life in a tiny drop of water. Up to this point, bright field microscopy relied on the absorption of transmitted light through the sample, which made it hardly possible to image transparent samples. Therefore, transparent samples were stained with dyes to enhance the contrast of the image. These dyes can also be molecularly specific, binding to specific targets like DNA, lipids, or carbohydrates, to highlight specific structures in biological tissue, but also proved to find their use in materials science, as will be shown in chapter 7 of this thesis. Chemically specific binding opened possibilities not only for imaging cell structure, but also for studying the chemical composition and chemical processes in cells.

At present, fluorescence is widely used as a major contrast generating approach. Fluorescent probes (or fluorophores) are chemically coupled to predefined antibodies and added to the sample, or can be even genetically encoded (fluorescent proteins) within the sample, to render specific domains against a fully dark background, giving a huge contrast enhancement. As will be discussed later on, fluorescence microscopy is also a powerful tool for a larger range of applications, which amongst other achievements, led to prominent insights into the cell's components and internal architecture. 


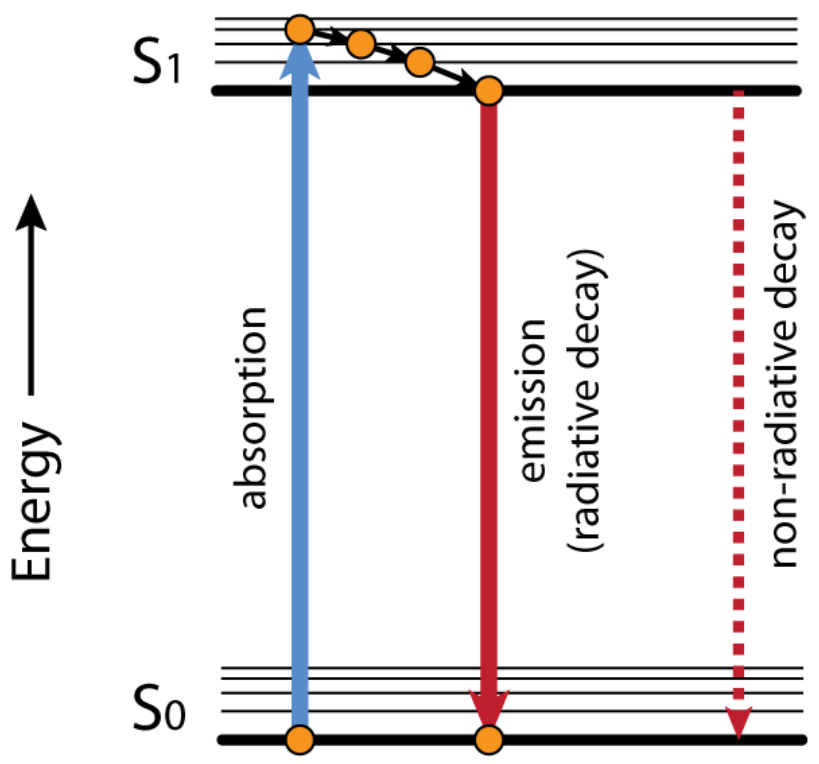

Figure 1.1 Jablonski diagram describing the energy process of fluorescence

\subsection{Fluorescence}

Fluorescence is an effect that was studied by Sir George Stokes [1]. He discovered that a fluorophore emits light that has a longer (red-shifted) wavelength compared with the wavelength of the absorbed light. The shift in wavelength, called Stokes shift, is the key to success for all fluorescence-based techniques. Using the appropriate filter-set, light from the illumination source can efficiently be brought to the sample, while at the same time emission light can efficiently be collected onto a detector. In addition, the filter-set filters out the illumination light at the detection channel, which renders the sample against a fully dark background.

To explain the energy process of fluorescence, often a so-called Jablonski energy diagram is used, as shown in Figure 1.1. The energy diagram shows a singlet ground state $\left(\mathrm{S}_{0}\right)$, which is the lowest-energy state of a fluorophore. At each state, due to the molecular conformation of the atoms in the fluorophore, a number of vibrational modes exist in which the atoms of the fluorophore can vibrate. These vibrational modes are indicated by the closely spaced energy levels. The fluorophore absorbs a high energy photon (with short wavelength), indicated by the blue arrow, and is excited from the singlet ground state to one of the vibrational levels of the 
singlet excited state $\left(\mathbf{S}_{1}\right)$. The fluorophore relaxes through vibrational relaxation to the excited state with the lowest energy, thereby losing energy and causing the Stokes shift. The fluorophore remains in this excited state for a characteristic time-period called fluorescence lifetime (explained below) before it decays back to the ground state. This decay can be either radiative or non-radiative. In case of radiative decay (solid red arrow) spontaneous emission occurs, thereby emitting a photon with less energy (longer wavelength and red-shifted) compared to the absorbed photon. Nonradiative decay (dashed red arrow) in general releases the energy as phonons, producing heat that is dissipated by the environment. The stochastic nature of the spontaneous emission classifies fluorescence as an incoherent process.

There are two generic ways to characterize the fluorescence process of fluorophores, spectrally-resolved and time-resolved, which are routinely performed on an ensemble of the fluorophore of interest. We developed a multimodal instrumentation platform, described in chapter 2, that can perform both spectrally and time-resolved characterisation, even for single (quantum) emitters. Later on, it will be made clear why it is so important to characterize single fluorophores.

\subsubsection{Spectroscopy}

As can be seen in the Jablonski diagram (Figure 1.1), the absorbance and emission intensity of the fluorophore depends on the energy, and thus on the wavelength of the light. In spectrally-resolved experiments, or spectroscopy in short, one measures the coupling strength between the ground state and excited state, for all transition frequencies.

Practically, an absorbance spectrum is measured by probing the relative amount of light that is absorbed by the ensemble of fluorophores, at each wavelength. The absorbance spectrum shows the coupling strength of all excitation transitions from the ground to excited state, and is often used to determine the optimal wavelength range to efficiently excite a fluorophore, or to identify a fluorophore within a sample.

Complementary to the absorbance spectrum, the emission spectrum is measured by probing the relative amount of emitted light at each wavelength, and represents the coupling strength of all emission transitions, from the excited state to the ground state. The emission spectrum is characteristic of the fluorophore, and is used for similar purposes as the absorbance spectrum. In addition, the emission spectrum is used in combination with the absorbance spectrum to, for example, find suitable donor-acceptor fluorophore pairs for FRET experiments (Förster resonant 
energy transfer [2-6]), or to measure the Stokes shift, which is sensitive to the environment of the fluorophores. We show in chapter 4 how we, for the first time, measured the Stokes shift of single fluorophores.

Excitation spectroscopy is similar to absorbance spectroscopy. Both absorbance and excitation spectroscopy require excitation wavelength scanning, but the main difference is the observable that is being measured. In excitation spectra the measured observable is the fluorescence emission, rather than the absorption of excitation light. The emission intensity is a measure of the absorbance, given the quantum efficiency (see next section) remains constant for all wavelengths. Therefore, the excitation spectrum in general produces the same spectral shape as the absorbance spectrum. To acquire an excitation spectrum, one scans the excitation wavelength while following the emission of the fluorophores. We developed single emitter excitation spectroscopy at room-temperature in chapter 3 , to access, for the first time, the spectral absorbance properties of single emitters across a broad spectral range.

\subsubsection{Time-resolved characterisation}

Time-resolved characterisation provides information about the residence time in the excited state and can also give access to the quantum efficiency of the fluorescent probes. As mentioned before, after a fluorophore relaxes to the excited state with the lowest energy, it resides there for an average characteristic time-period called the lifetime of the excited state, until a spontaneous process occurs and the fluorophore decays back to the ground state. Spontaneous emission is a purely quantum mechanical phenomenon, in which a quantum emitter has a probability to couple the energy in the excited state to an electromagnetic field. The spontaneous emission rate can be derived using perturbation theory and is described by Fermi's golden rule [7],

$$
\gamma_{i \rightarrow[f]}=\frac{2 \pi}{\hbar^{2}}\left|\left\langle f\left|H^{\prime}\right| i\right\rangle\right|^{2} \delta\left(\omega_{f i}-\omega_{0}\right)
$$

where $\gamma_{i \rightarrow[f]}$ is the rate of the transition, $\hbar$ is Planck's constant, $f$ and $i$ the final and initial state, $H^{\prime}$ the perturbing Hamiltonian, $\omega_{f i}$ the frequency of the transition and $\omega_{0}$ the frequency of the driving field. Fermi's golden rule can also be written in terms of dipole moment $\boldsymbol{d}$ and driving field $\boldsymbol{E}_{\mathbf{0}}$ [8]: 


$$
\gamma_{i \rightarrow[f]}=\frac{\pi}{2 \hbar^{2}} \sum_{[f]}\left|\left(\boldsymbol{d} \cdot \boldsymbol{E}_{\mathbf{0}}\right)_{f i}\right|^{2} \delta\left(\omega_{f i}-\omega_{0}\right)
$$

After summing over all free electromagnetic field modes, equation 1.2 can be simplified to [9]:

$$
\gamma_{\text {rad }}=\frac{\omega_{f i}^{3} n|\boldsymbol{d}|^{2}}{3 \pi \hbar \varepsilon_{0} c^{3}}
$$

where $n$ is the refractive index, $\varepsilon_{0}$ the vacuum permittivity, and $c$ the speed of light in vacuum. The spontaneous emission process is a stochastic process, and typically shows excited state residence times that are distributed by an exponentially decreasing probability function. The lifetime $\tau$ is the time-constant of this exponential decay function. Since the spontaneous decay process is by far the slowest step in the fluorescence process, the decay rate $(\gamma=1 / \tau)$ indicates the average rate of the fluorophore decaying from the excited state back to the ground state.

In practice, the lifetime can be measured by recording time-delays between excitation pulses and time-of-arrival of detected fluorescence photons (described in the next chapter). After collecting significant statistics on the time-of-arrival of the detected fluorescence photons with respect to the excitation pulses, the resulting decay distribution is typically fitted with an exponential decay function to obtain the lifetime of the fluorophore. The fluorescence lifetime of fluorophores is typically at the nanosecond-scale range, which is long compared to various fluctuations occurring in the direct nano-environment of the emitter. This makes the lifetime a sensitive parameter to probe interactions of the fluorophore with its environment that occur within the time-span of the lifetime. For example, the lifetime can be used to measure diffusion of oxygen molecules, rotational diffusion, solvent polarity, FRET coupling and quenching [1].

Furthermore, time-resolved measurements allow for the determination of the quantum efficiency. The quantum efficiency $(\mathrm{QE})$ is classically described as the ratio of the number of emitted photons to the number of absorbed photons (equation 1.4). Obviously, fluorophores with a high QE are preferably used in fluorescence microscopy and spectroscopy.

$$
Q E=\frac{N_{\text {emitted }}}{N_{\text {absorbed }}}=\frac{\gamma_{\text {rad }}}{\gamma_{\text {rad }}+\gamma_{\text {nrad }}}
$$


Conventionally, the $\mathrm{QE}$ is measured by comparing the fluorescence emission intensity of the fluorophore of interest with that of a reference fluorophore with identical absorbance at the same excitation wavelength. However, this method is not sufficiently accurate, because it always underestimates the true $\mathrm{QE}$ due to photobleaching and blinking of fluorophores. This underestimation can be solved by determining the $\mathrm{QE}$ through time-resolved measurements, where the $\mathrm{QE}$ can be described as the relative number of decay processes occurring radiatively, compared to the total number of decays (equation 1.4). Chapter 7 discusses a new approach that resolves the radiative and non-radiative decay rates from the total decay rate (inverse of the measured fluorescence lifetime), by inducing controlled modifications to the local photonic environment [10-12], modulating solely the radiative decay channel.

\subsection{Photophysics of single emitters}

The photophysics that is observed on an ensemble of fluorophores is averaged over many single fluorophores, each having its own individual properties. Photobleaching is an example of a single-emitter feature that, on the ensemble level, is observed as a monotonic decrease in fluorescence emission intensity, which is detrimental for imaging applications, because the image contrast reduces over time. A trade-off is often required between contrast and observation time, due to photobleaching of the fluorophores. On the single emitter level, photobleaching is observed as a single fluorophore is suddenly making a transition to a dark state, or is destroyed, and does not emit light anymore.

A more interesting feature of single emitters is emission intermittency - in short, blinking. Blinking is the sudden switching of an emitter between emitting and non-emitting states [13-16], observed as a binary emission intensity fluctuation. The coexistence of the non-emitting states reduces the fluorescence duty cycle and effectively lowers the efficiency of the fluorophores while still absorbing photons. Therefore, blinking is seen as a burden in many application fields. Since blinking averages out for ensembles of emitters and is thus undetectable, blinking was only discovered with the development of single molecule detection methods [17]. Since then, blinking has been found for many emitter classes [18-25]. Interestingly, the underlying mechanism of blinking varies between emitter classes. Although the exact nature of blinking is still being widely debated [26], both blinking and bleaching already play an essential role in recently developed super-resolution microscopy techniques like STORM, PALM, RESOLFT, and BLINK [27-33]. 
In chapters 5 and 6 , we study blinking behavior of single semiconductor nanocrystals (quantum dots) in detail, and use our multimodal platform to develop single emitter techniques that support the characterization of the interesting state switching behavior of these single emitters, giving new insights into the blinking mechanism.

The rapid development of these new microscopy techniques ask for new fluorescence probes, which in turn requires novel characterization methods for studying the photophysics of these new probes at the single emitter scale. Therefore, optical single molecule fluorescence studies are becoming increasingly popular for studying complex systems at the nanoscale [34-38]. Since the first demonstration of single emitter fluorescence spectroscopy over two decades ago, techniques to detect and characterize the emission from single emitters have become increasingly sophisticated and versatile [39-41]. These developments have made optical single emitter spectroscopy an indispensable tool to address complex problems in chemistry [42, 43], the material sciences [44-46], and the life sciences [47-54].

Over time, more and more of the photophysical parameters that can be probed at the ensemble level have become accessible on the single molecule level. Currently, the evolution of intensity over time [55], the absorption [56-59], the lifetime [60], the polarization [61], and the emission spectra [62-64] of single emitters are readily accessible on the single emitter level. Studying these different parameters on the single molecule level, which avoids the averaging effects inherent to studying ensembles, gave fundamental new insights into the photophysics of various classes of emitters. Recently, we succeeded in recording excitation spectra of single emitters [65], which we first demonstrated on single quantum dots (see chapter 3) and later on single organic fluorophores (see chapter 4). Moreover, by recording both the excitation and emission spectra of the single organic fluorophores, we have been able to study the Stokes shift of single organic emitters (see chapter 4).

Throughout this thesis, various techniques and methods are described that we developed to characterize single emitters, and to expand knowledge on the photophysics of quantum emitting systems.

\subsection{Fluorescent probes used in the thesis}

A wide set of fluorescent probes is currently commercially available [27, 31, 40, 66], each fluorophore having their own range of applications. Accordingly, we chose fluorophores that suited best for the topics in this thesis for reasons that are listed below. 


\subsubsection{Quantum dots}

Semiconductor nanocrystals, or quantum dots, have unique optical properties [6770], including a broad absorption range, a narrow luminescence emission spectrum, size-dependent spectral properties [71] and significantly enhanced photostability compared to organic fluorophores. These properties do not exist for bulk semiconductor materials, and are only expressed when the semiconductor material becomes so small that quantum confinement occurs. The energy of an absorbed photon is used to separate charges across the band-gap, creating an electron-hole pair (exciton) that is confined to the nanocrystal. It is precisely this quantum confinement which makes the emission photon energy of the quantum dot tunable with size. A smaller charge confinement results in states with higher energy according to Schrödinger's equation in quantum theory, in turn causing blue-shifted spectral properties. Furthermore, quantum dots can be considered as three-dimensional quantum wells, which exhibit an increasing density of states for higher photon energies. The higher density of states increases the absorption probability for photons with higher energy, creating a so-called quasi-continuum of states in the spectral properties of the nanocrystals. This quasi-continuum provides a broad photon absorption range. At the same time quantum dots have a narrow emission band, because both the electron and hole relax to the energy levels that are closest to the bandgap, before recombining. Furthermore, quantum dots have a crystalline structure, which makes them inert to environmental degradation, and makes them very photostable. These feasible properties make quantum dots promising nanomaterials in various fields of research ranging from in-vivo probes in the lifesciences [72-76] to single photon light sources in telecommunications [77], solar cells [78], LEDs [79] or quantum computing [80, 81]. In chapter 3, we make use of the broad absorption range and enhanced photostability of the quantum dots to develop a platform that allows for excitation spectroscopy of single emitters. The excitation spectra give access to the spectral absorbance properties of single emitters.

\subsubsection{Perylene dyes}

Perylene is an organic dye and is one of the brightest and most stable dye of the organic fluorophore class, and is therefore applicable in a variety of fields, from life sciences $[82,83]$ to organic solar cells $[84,85]$ and organic light-emitting-diodes (OLEDs) [86]. Side-groups are often attached to the perylene structure, allowing for easy chemical modification and functionalization. We utilized side-groups for controlled surface patterning [87] (see chapter 7). 
Moreover, perylene dyes show an exceedingly rich photophysical behavior at the single emitter level. The emission spectra of the single emitters show spectral diffusion [88, 89], meaning that the energy scheme of the emitter changes over time, which in turn causes changes in the emission spectra. Spectral diffusion is, for example, caused by variation in molecular conformation of the fluorophore itself, or by changes in the local environment of the emitter. The alterations to the energy scheme should, however, not solely be visible in the emission spectra, but also in the excitation spectra, which has not yet been reported in literature. Moreover, it made us wonder whether the Stokes shift remains constant at the single emitter level, since it has always been assumed that the Stokes shift is a constant parameter for each fluorophore. Since in the course of this work we built a platform capable of recording both emission (chapter 2) and excitation spectra (chapter 3) of single emitters, we are able to answer both of these questions in chapter 4 .

\subsubsection{DsRed (Red fluorescent protein)}

Fluorescent proteins (FPs) are biological fluorophores that can be genetically encoded into biological cells to target and highlight specific domains, which makes them popular fluorescence tools in life sciences. One of the most popular FPs is the green fluorescent protein (GFP) with its main derivatives blue (BFP), cyan (CFP), and yellow (YFP) fluorescent protein [90]. Red FPs have been developed, but often yielded red FPs with a low extinction coefficient and low quantum efficiency. DsRed was one of the first bright red emitting FPs. DsRed is a tetrameric fluorescent protein, randomly composed of green and red emitting monomers, and shows enhanced red emission due to efficient FRET coupling from the green to the red monomer [91], which makes DsRed a bright and biologically compatible fluorophore. In chapter 7 of this thesis, we study DsRed patterns, which are nanodispensed (NADIS) with an AFM cantilever to produce micro-scale sized droplets in a defined pattern [92].

\subsection{Thesis overview}

- Chapter 2 describes the design and assembly of the instrumentation that was used as a platform to study photophysics of single emitters.

- Chapter 3 reports on the way we measured for the first time excitation spectra of single emitters (quantum dots) over a broad spectral range and at room temperature, giving access to the spectral absorbance properties of single emitters. 
- Chapter 4 expands the capabilities of the method described in chapter 3 by measuring excitation spectra of single organic fluorophores. In addition, we were able to record both the excitation and emission spectrum of the same emitter, which gives access to the Stokes shift of single emitters.

- Chapters 5 and 6 study the blinking characteristics of quantum dots. Chapter 5 studies the effect of the excitation wavelength on blinking behavior, which could be utilized to optimize fluorophore performance either for conventional or for super-resolution microscopy. Chapter 6 studies the blinking behavior of quantum dots with high time resolution, revealing a defined set of states for each quantum dot at shorter time scales than used in earlier reports in the literature. In this chapter, a refinement of the commonly used trapped charge model is proposed to explain our observations.

- The preceding chapters focus on the development and application of an advanced single-molecule spectroscopy platform to characterize photophysics of single emitters. Chapter 7 discusses potential future applications using the same platform. One example highlights a new method to accurately quantify the quantum efficiency of fluorophores, and the other example shows how the instrumentation in this chapter was used to visualize and characterize surfaces that are patterned by either chemical binding (thiol-ene click reaction) or by physical nano-dispensed (NADIS) droplets of fluorophores using an AFM cantilever.

\section{REFERENCES}

1. $\quad$ Lakowicz, J.R., Principles of fluorescence spectroscopy. 3rd ed. 2006.

2. Kang, G.P., et al., Forster resonance energy transfer as a probe of membrane protein folding. Biochimica Et Biophysica Acta-Biomembranes, 2012. 1818(2): p. 154-161.

3. Tisler, J., et al., Highly efficient fret from a single nitrogen-vacancy center in nanodiamonds to a single organic molecule. Acs Nano, 2011. 5(10): p. 7893-7898.

4. Borsch, M. and J. Wrachtrup, Improving fret-based monitoring of single chemomechanical rotary motors at work. Chemphyschem, 2011. 12(3): p. 542-553.

5. Buning, R. and J. van Noort, Single-pair fret experiments on nucleosome conformational dynamics. Biochimie, 2010. 92(12): p. 1729-1740.

6. Medintz, I.L. and H. Mattoussi, Quantum dot-based resonance energy transfer and its growing application in biology. Physical Chemistry Chemical Physics, 2009. 11(1): p. $17-45$. 
7. Fermi, E. and J. Orear, Nuclear physics: A course given by enrico fermi at the university of chicago. 1950: University of Chicago Press.

8. Gerry, C.C. and P.L. Knight, Introductory quantum optics. 2005: Cambridge university press.

9. Lodahl, P., et al., Controlling the dynamics of spontaneous emission from quantum dots by photonic crystals. Nature, 2004. 430(7000): p. 654-657.

10. Drexhage, K.H., Influence of a dielectric interface on fluorescence decay time. Journal of Luminescence, 1970. 1-2(0): p. 693-701.

11. Bär, S., et al., Microcavities: Tailoring the optical properties of single quantum emitters. Analytical and Bioanalytical Chemistry, 2010. 396(1): p. 3-14.

12. Cesa, Y., et al., Manipulation of the local density of photonic states to elucidate fluorescent protein emission rates. Physical Chemistry Chemical Physics, 2009. 11(14): p. 2525-2531.

13. Cook, R.J. and H.J. Kimble, Possibility of direct observation of quantum jumps. Physical Review Letters, 1985. 54(10): p. 1023-1026.

14. Nirmal, M., et al., Fluorescence intermittency in single cadmium selenide nanocrystals. Nature, 1996. 383(6603): p. 802-804.

15. Shimizu, K.T., et al., Blinking statistics in single semiconductor nanocrystal quantum dots. Physical Review B, 2001. 63(20).

16. Stopel, M.H.W., et al., Blinking statistics of colloidal quantum dots at different excitation wavelengths. RSC Advances, 2013. 3: p. 17440-17445.

17. Sauter, T., et al., Observation of quantum jumps. Physical Review Letters, 1986. 57(14): p. 1696-1698.

18. Patel, S.A., et al., Electron transfer-induced blinking in ag nanodot fluorescence. Journal of Physical Chemistry C, 2009. 113(47): p. 20264-20270.

19. Orrit, M., Chemical and physical aspects of charge transfer in the fluorescence intermittency of single molecules and quantum dots. Photochemical \& Photobiological Sciences, 2010. 9(5): p. 637-642.

20. Cichos, F., C. von Borczyskowski, and M. Orrit, Power-law intermittency of single emitters. Current Opinion in Colloid \& Interface Science, 2007. 12(6): p. 272-284.

21. Hoogenboom, J.P., et al., Power-law blinking in the fluorescence of single organic molecules. Chemphyschem, 2007. 8(6): p. 823-833.

22. Stefani, F.D., J.P. Hoogenboom, and E. Barkai, Beyond quantum jumps: Blinking nanoscale light emitters. Physics Today, 2009. 62(2): p. 34-39.

23. Zondervan, R., et al., Photoblinking of rhodamine $6 \mathrm{~g}$ in poly(vinyl alcohol): Radical dark state formed through the triplet. Journal of Physical Chemistry A, 2003. 107(35): p. 6770-6776.

24. Stracke, F., et al., Correlation of emission intensity and spectral diffusion in room temperature single-molecule spectroscopy. Chemphyschem, 2005. 6(7): p. 12421246.

25. Blum, C. and V. Subramaniam, Single-molecule spectroscopy of fluorescent proteins. Analytical and Bioanalytical Chemistry, 2009. 393(2): p. 527-541.

26. Frantsuzov, P., et al., Universal emission intermittency in quantum dots, nanorods and nanowires. Nature Physics, 2008. 4(7): p. 519-522.

27. Bates, M., et al., Multicolor super-resolution imaging with photo-switchable fluorescent probes. Science, 2007. 317(5845): p. 1749-1753. 
28. de Lange, F., et al., Cell biology beyond the diffraction limit: Near-field scanning optical microscopy. Journal of Cell Science, 2001. 114(23): p. 4153-4160.

29. Huang, B., M. Bates, and X.W. Zhuang, Super-resolution fluorescence microscopy, in Annual review of biochemistry. 2009, Annual Reviews: Palo Alto. p. 993-1016.

30. Huang, B., et al., Three-dimensional super-resolution imaging by stochastic optical reconstruction microscopy. Science, 2008. 319(5864): p. 810-813.

31. Vogelsang, J., et al., Make them blink: Probes for super-resolution microscopy. Chemphyschem, 2010. 11(12): p. 2475-2490.

32. Cordes, T., et al., Resolving single-molecule assembled patterns with superresolution blink-microscopy. Nano Letters, 2010. 10(2): p. 645-651.

33. Steinhauer, C., M.S. Itano, and P. Tinnefeld, Super-resolution fluorescence imaging with blink microscopy. 2013. p. 111-129.

34. Roeffaers, M.B.J., et al., Single-molecule fluorescence spectroscopy in (bio)catalysis. Proceedings of the National Academy of Sciences of the United States of America, 2007. 104(31): p. 12603-12609.

35. Zuchner, T., A.V. Failla, and A.J. Meixner, Light microscopy with doughnut modes: A concept to detect, characterize, and manipulate individual nanoobjects. Angewandte Chemie-International Edition, 2011. 50(23): p. 5274-5293.

36. Kawashima, N., et al., Reversible dimerization of egfr revealed by single-molecule fluorescence imaging using quantum dots. Chemistry-a European Journal, 2010. 16(4): p. 1186-1192.

37. Mueller, V., et al., Sted nanoscopy reveals molecular details of cholesterol- and cytoskeleton-modulated lipid interactions in living cells. Biophysical Journal, 2011. 101(7): p. 1651-1660.

38. Watanabe, S., et al., Protein localization in electron micrographs using fluorescence nanoscopy. Nature Methods, 2011. 8(1): p. 80-U117.

39. Cotlet, M., et al., Identification of different emitting species in the red fluorescent protein dsred by means of ensemble and single-molecule spectroscopy. Proceedings of the National Academy of Sciences of the United States of America, 2001. 98(25): p. $14398-14403$.

40. Ha, T. and P. Tinnefeld, Photophysics of fluorescent probes for single-molecule biophysics and super-resolution imaging, in Annual review of physical chemistry, vol 63, M.A. Johnson and T.J. Martinez, Editors. 2012, Annual Reviews: Palo Alto. p. 595-617.

41. Hinkeldey, B., A. Schmitt, and G. Jung, Comparative photostability studies of bodipy and fluorescein dyes by using fluorescence correlation spectroscopy. Chemphyschem, 2008. 9(14): p. 2019-2027.

42. Moerner, W.E., $A$ dozen years of single-molecule spectroscopy in physics, chemistry, and biophysics. The Journal of Physical Chemistry B, 2002. 106(5): p. 910-927.

43. Tinnefeld, P. and M. Sauer, Branching out of single-molecule fluorescence spectroscopy: Challenges for chemistry and influence on biology. Angewandte Chemie International Edition, 2005. 44(18): p. 2642-2671.

44. Lupton, J.M., Single-molecule spectroscopy for plastic electronics: Materials analysis from the bottom-up. Advanced Materials, 2010. 22(15): p. 1689-1721. 
45. Roeffaers, M.B.J., et al., Spatially resolved observation of crystal-face-dependent catalysis by single turnover counting. Nature, 2006. 439(7076): p. 572-575.

46. Wöll, D., et al., Radical polymerization tracked by single molecule spectroscopy. Angewandte Chemie International Edition, 2008. 47(4): p. 783-787.

47. Dertinger, T., et al., Two-focus fluorescence correlation spectroscopy: A new tool for accurate and absolute diffusion measurements. Chemphyschem, 2007. 8(3): p. 433-443.

48. Moerner, W.E., New directions in single-molecule imaging and analysis. Proceedings of the National Academy of Sciences, 2007. 104(31): p. 12596-12602.

49. Schuler, B., Single-molecule fluorescence spectroscopy of protein folding. Chemphyschem, 2005. 6(7): p. 1206-1220.

50. Weckhuysen, B.M., Chemical imaging of spatial heterogeneities in catalytic solids at different length and time scales. Angewandte Chemie-International Edition, 2009. 48(27): p. 4910-4943.

51. Moerner, W.E., A dozen years of single-molecule spectroscopy in physics, chemistry, and biophysics. Journal of Physical Chemistry B, 2002. 106(5): p. 910927.

52. Cai, L., N. Friedman, and X.S. Xie, Stochastic protein expression in individual cells at the single molecule level. Nature, 2006. 440(7082): p. 358-362.

53. Forget, A.L. and S.C. Kowalczykowski, Single-molecule imaging of DNA pairing by reca reveals a three-dimensional homology search. Nature, 2012. 482(7385): p. 423U178.

54. Rybina, A., et al., Distinguishing alternative reaction pathways by single-molecule fluorescence spectroscopy. Angewandte Chemie-International Edition, 2013. 52(24): p. $6322-6325$.

55. Xie, X.S. and R.C. Dunn, Probing single-molecule dynamics. Science, 1994. 265(5170): p. 361-364.

56. Vermeulen, P., L. Cognet, and B. Lounis, Photothermal microscopy: Optical detection of small absorbers in scattering environments. Journal of Microscopy, 2014. 254(3): p. 115-121.

57. Chong, S., W. Min, and X.S. Xie, Ground-state depletion microscopy: Detection sensitivity of single-molecule optical absorption at room temperature. The Journal of Physical Chemistry Letters, 2010. 1(23): p. 3316-3322.

58. Gaiduk, A., et al., Room-temperature detection of a single molecule's absorption by photothermal contrast. Science, 2010. 330(6002): p. 353-356.

59. Kukura, P., et al., Single-molecule sensitivity in optical absorption at room temperature. The Journal of Physical Chemistry Letters, 2010. 1(23): p. 3323-3327.

60. Gomez, D.E., et al., Exciton-trion transitions in single cdse-cds core-shell nanocrystals. Acs Nano, 2009. 3(8): p. 2281-2287.

61. Warshaw, D.M., et al., Myosin conformational states determined by single fluorophore polarization. Proceedings of the National Academy of Sciences of the United States of America, 1998. 95(14): p. 8034-8039.

62. Schleifenbaum, F., et al., Single-molecule spectral dynamics at room temperature. Molecular Physics, 2009. 107(18): p. 1923-1942.

63. Blum, C., A.J. Meixner, and V. Subramaniam, Room temperature spectrally resolved single-molecule spectroscopy reveals new spectral forms and photophysical 
versatility of aequorea green fluorescent protein variants. Biophysical Journal, 2004. 87(6): p. 4172-4179.

64. Blum, C., A.J. Meixner, and V. Subramaniam, Dark proteins disturb multichromophore coupling in tetrameric fluorescent proteins. Journal of Biophotonics, 2011. 4(1-2): p. 114-121.

65. Blum, C., et al., Room temperature excitation spectroscopy of single quantum dots. Beilstein Journal of Nanotechnology, 2011. 2: p. 516-524.

66. Heilemann, M., et al., Super-resolution imaging with small organic fluorophores. Angewandte Chemie International Edition, 2009. 48(37): p. 6903-6908.

67. Knappenberger, K.L., et al., Excitation wavelength dependence of fluorescence intermittency in cdse/zns core/shell quantum' dots. Nano Letters, 2007. 7(12): p. 3869-3874.

68. Neuhauser, R.G., et al., Correlation between fluorescence intermittency and spectral diffusion in single semiconductor quantum dots. Physical Review Letters, 2000. 85(15): p. 3301-3304.

69. Norris, D.J. and M.G. Bawendi, Measurement and assignment of the sizedependent optical spectrum in cdse quantum dots. Physical Review B, 1996. 53(24): p. $16338-16346$.

70. Zwiller, V., et al., Single quantum dots emit single photons at a time: Antibunching. experiments. Applied Physics Letters, 2001. 78(17): p. 2476-2478.

71. Alivisatos, A.P., Semiconductor clusters, nanocrystals, and quantum dots. Science, 1996. 271(5251): p. 933-937.

72. Alivisatos, A.P., W. Gu, and C. Larabell, Quantum dots as cellular probes. Annual Review of Biomedical Engineering, 2005. 7(1): p. 55-76.

73. Medintz, I.L., et al., Quantum dot bioconjugates for imaging, labelling and sensing. Nature Materials, 2005. 4(6): p. 435-446.

74. Michalet, X., et al., Quantum dots for live cells, in vivo imaging, and diagnostics. Science, 2005. 307(5709): p. 538-544.

75. Chan, W.C.W., et al., Luminescent quantum dots for multiplexed biological detection and imaging. Current Opinion in Biotechnology, 2002. 13(1): p. 40-46.

76. Chien, F.-C., C.W. Kuo, and P. Chen, Localization imaging using blinking quantum dots. Analyst, 2011. 136(8).

77. Ward, M.B., et al., Electrically driven telecommunication wavelength single-photon source. Applied Physics Letters, 2007. 90(6): p. -.

78. Gratzel, M., Solar energy conversion by dye-sensitized photovoltaic cells. Inorganic Chemistry, 2005. 44(20): p. 6841-6851.

79. Coe, S., et al., Electroluminescence from single monolayers of nanocrystals in molecular organic devices. Nature, 2002. 420(6917): p. 800-803.

80. Kroutvar, M., et al., Optically programmable electron spin memory using semiconductor quantum dots. Nature, 2004. 432(7013): p. 81-84.

81. Loss, D. and D.P. DiVincenzo, Quantum computation with quantum dots. Physical Review A, 1998. 57(1): p. 120-126.

82. Céspedes-Guirao, F.J., et al., $A$ water-soluble perylene dye functionalised with a 17 $\beta$-estradiol: A new fluorescent tool for steroid hormones. Chemical Communications, 2011. 47(29): p. 8307-8309. 
83. Zhao, Y., et al., Water-soluble 3,4:9,10-perylene tetracarboxylic ammonium as a high-performance fluorochrome for living cells staining. Luminescence, 2009. 24(3): p. $140-143$.

84. Kozma, E. and M. Catellani, Perylene dïmides based materials for organic solar cells. Dyes and Pigments, 2013. 98(1): p. 160-179.

85. Kim, Y. and E. Lim, Development of polymer acceptors for organic photovoltaic cells. Polymers, 2014. 6(1): p. 382-407.

86. Pu, Y.J., et al., Solution-processable organic fluorescent dyes for multicolor emission in organic light emitting diodes. Journal of Materials Chemistry, 2008. 18(35): p. 4183-4188.

87. Wasserberg, D., et al., Patterning perylenes on surfaces using thiol-ene chemistry. Journal of Materials Chemistry, 2012. 22(32): p. 16606-16610.

88. Blum, C., et al., Discrimination and interpretation of spectral phenomena by roomtemperature single-molecule spectroscopy. Journal of Physical Chemistry A, 2001. 105(29): p. 6983-6990.

89. Stracke, F., et al., Intrinsic conformer jumps observed by single molecule spectroscopy in real time. Chemical Physics Letters, 2000. 325(1-3): p. 196-202.

90. Campbell, R.E., et al., A monomeric red fluorescent protein. Proceedings of the National Academy of Sciences, 2002. 99(12): p. 7877-7882.

91. Schleifenbaum, F., et al., New insights into the photophysics of dsred by multiparameter spectroscopy on single proteins. The Journal of Physical Chemistry B, 2008. 112(25): p. 7669-7674.

92. Fang, A., E. Dujardin, and T. Ondarçuhu, An approach to control of droplet size in nanoscale dispensing: Journal of Physics: Conference Series, 2007. 61(1): p. 298. 



\title{
Chapter 2
}

\section{Multimodal fluorescence imaging spectroscopy}

\begin{abstract}
Multimodal fluorescence imaging is a versatile method that has a wide application range from biological studies to materials science. Typical observables in multimodal fluorescence imaging are fluorescence intensity, lifetime, polarization, and excitation and emission spectra, which are recorded at chosen locations at the sample. This chapter describes the instrumentation that allows for multimodal fluorescence imaging, which is used as a platform to study photophysics of single emitters, and explains the corresponding data analysis procedures for the observables.
\end{abstract}

This chapter has been published as Chapter 23 in the book - Fluorescence Spectroscopy and Microscopy: Methods and Protocols, Multimodal Fluorescence Imaging Spectroscopy, Methods in Molecular Biology Volume 1076, pages 521-536, Springer Protocols 2014 


\subsection{Introduction}

Fluorescence is characterized by the absorption of light, followed by the spontaneous emission of light that is typically 'red-shifted'. The red-shifted light can be easily separated from the illumination light by choosing a suitable filter set. In fluorescence microscopy, this spectral separation of absorbed and emitted light is exploited to highlight and study specific domains spatially, rendered visible against a dark background. Nevertheless, these methods are of limited use in their ability to analyze the dynamics, interactions and physical environment of molecules when unassisted by spectroscopy. Imaging spectroscopy methods enable the extension of simple spatial analyses to demonstrate function, co-localization and molecular interaction at the nano-scale [1-4].

Multimodal imaging spectroscopy combines imaging with a range of spectroscopic observables to image microscopic objects, while at the same time obtaining detailed physico-chemical information at the nano-scale. The parameters that are typically measured are fluorescence intensity, lifetime [5-7], polarization [810], and emission spectra [11-13]. At the moment, there is an upcoming interest in super-continuum (SC) white light sources [14-16]. These light sources provide the flexibility to pick any desired excitation wavelength between $400 \mathrm{~nm}$ and $>2000 \mathrm{~nm}$ and allow for Time-Correlated Single Photon Counting (TCSPC) experiments to accurately measure fluorescence lifetime. Even more important is that SC light sources give the possibility to perform excitation spectroscopy [15-17], in addition to the commonly measured observables in multimodal microscopy mentioned above. In this chapter, it is described how to assemble and operate a versatile multimodal fluorescence lifetime imaging microscopy (FLIM) setup and discuss important practical bottlenecks that are crucial for optimum functionality of the setup.

\subsection{Instrumentation}

There are many ways to implement multimodal imaging spectroscopy. This chapter describes an instrument configuration that is capable of both wide-field fluorescence imaging and single-emitter confocal imaging spectroscopy, although the latter method will be the main focus of this chapter. The instrumentation incorporates a supercontinuum white light laser in combination with an acousto-optical tunable filter (AOTF) to provide any desired excitation wavelength in the range between $400 \mathrm{~nm}$ up to $2000 \mathrm{~nm}$, and allows for fast switching between different wavelengths within the full visible range. The use of a high NA infinity corrected imaging objective lies at the heart of the versatility of the setup, allowing for easy add-on of different detector types to the detection path, extending the multimodality of the setup. The confocal 
detectors have a sensitivity that is capable of detecting single photons, which not only provides a high signal-to-background ratio, but also allows one to study single emitting fluorophores (quantum emitters). The complete setup scheme for the multimodal fluorescence lifetime imaging setup is shown in Figure 2.1. The full assembly procedure of such an imaging setup was published as a book chapter in Fluorescence Spectroscopy and Microscopy [18] and will be briefly explained in this chapter.

\subsection{Wide-field microscopy}

\subsubsection{Illumination}

Wide-field illumination can be achieved either by standard transmission illumination by a light source mounted on the illumination pillar above the sample stage or by an epi-illumination configuration through the back port of the microscope. This combination enables working with transparent as well as non-transparent substrates or samples. Köhler illumination is a commonly used illumination scheme in light microscopy to achieve a homogeneous illumination profile across the full field-ofview $(\mathrm{FoV})$ and is usually not considered as a critical alignment procedure. The sources that are used for wide-field illumination are preferably light sources with low coherence, because coherent sources cause interference at the illumination pattern. For coherent light sources, one should implement additional rotating diffusive optical elements to scramble the phase of the coherent light. For both high light intensity and good applicability for fluorescence, it is recommended to use high power LEDs or a Mercury lamp.

\subsubsection{Imaging}

The imaging CCD is used to obtain a picture of a full area at one instance. A slice of the plant Convallaria Majalis (Lily of the valley, Figure 2.2) is a good sample to test the alignment and functionality of both the wide-field and the confocal detectors, because it is highly auto-fluorescent and shows a large spectral diversity. A proper filter set should be used to spectrally separate the excitation light (typically a bandpass filter) and emission light (typically a long-pass filter). A good all-round filter combination is the FF02-447/60 band-pass filter for excitation light and the BLP488R long-pass filter for emission light (both Semrock filters), since there is a wide collection of dyes that can be excited with blue light. 


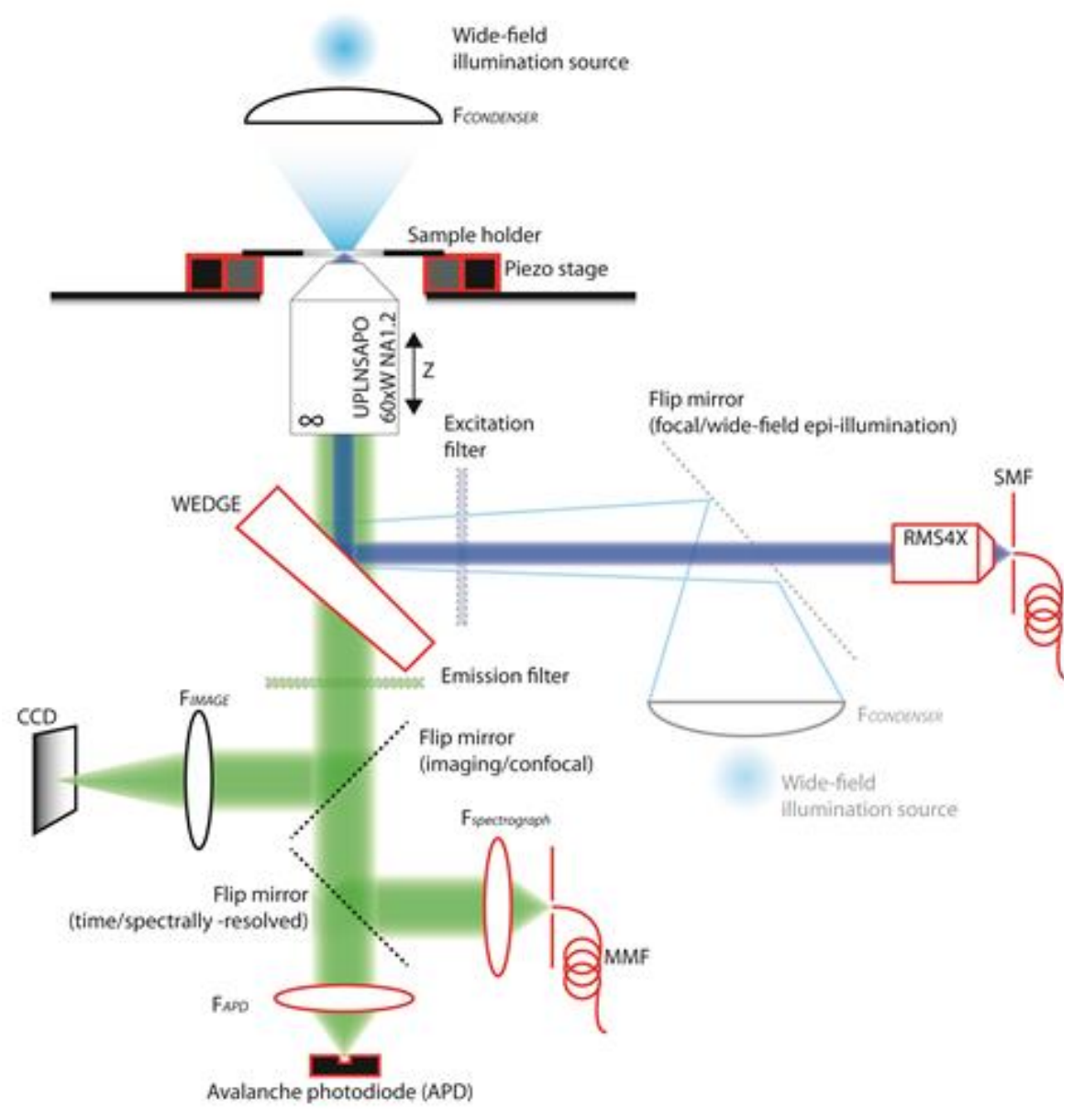

Figure 2.1 Setup scheme for the multimodal fluorescence lifetime imaging setup. The parts that are highlighted in red are parts that are essential for the confocal illumination and detection configuration. Without these highlighted components, the setup would operate in conventional fluorescence wide-field imaging configuration. Adding the highlighted components will not degrade the performance in the wide-field imaging configuration. A wide-field illumination source is depicted in this figure for the conventional transmission illumination, but the same light source can be used as well to provide epi-illumination (shaded) through the same light path as the confocal illumination, when focused on the back focal plane of the imaging microscope objective. Filters should be added to this scheme to spectrally separate excitation and emission light. Excitation filters should be placed after the excitation source (wide-field illumination or confocal illumination) and the emission filters should be placed after the wedge. 

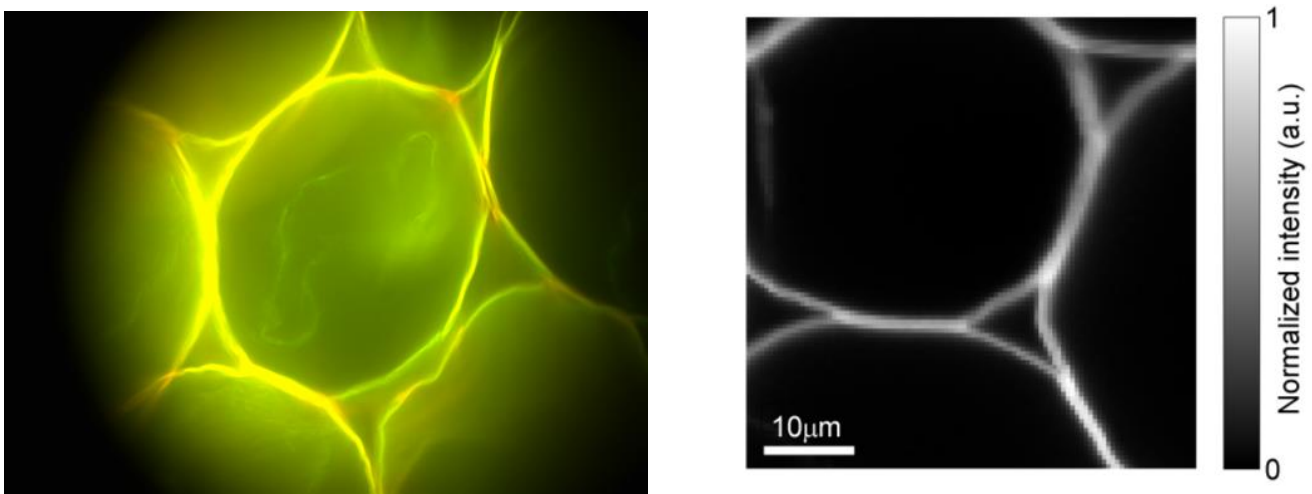

Figure 2.2 (left) Wide-field fluorescence image of Convallaria Majalis (Lily-of-the-valley). The typical size of a cell is around $30 \mu \mathrm{m}$. This sample is highly auto-fluorescent and provides a broad spectral diversity, for example observed by the emission color variation along the cell wall, which is beneficial for testing the functionality of the multimodal setup. (right) Confocal intensity map of Convallaria Majalis.

\subsection{Confocal spectroscopy}

\subsubsection{Excitation source}

The three most prominent requirements for the excitation source are pulse capability, spectral versatility and the ability to focus to a diffraction-limited spot. The Fianium SC400-PP super-continuum excitation source used here suits all three requirements. In addition, the laser's output power is high (2W total and about $1 \mathrm{~mW} / \mathrm{nm}$ in the visible range), allowing for single emitter experiments, and even opens a path to single emitter excitation spectroscopy, as shown in the next chapter.

To exploit the spectral versatility of the source, an acousto-optical tunable filter (AOTF) is implemented to select any desired excitation wavelength in the visible range with a narrow band (3-10nm from blue to red, respectively). A fixed radio frequency $(\mathrm{RF})$ is applied to create a standing wave pattern inside the AOTF crystal, causing diffraction of light. At the back-end of the crystal, one can find multiple diffractive orders where only one of the first order beams is collinear with the crystal and does not displace when the AOTF is tuned to a different wavelength. This beam has a high intensity within a narrow band at the desired wavelength and suppresses the light outside this band. The calibration of the AOTF allows one to choose any desired excitation wavelength and opens up possibilities for excitation spectroscopy and hyperspectral imaging. Calibration of the AOTF is done by applying different RF frequencies to the crystal and measuring the corresponding transmission wavelengths using a calibrated spectrograph (Thorlabs CCS100/M). 
The peak transmission wavelength as a function of RF frequency is the required calibration curve and should be fitted with a cubic polynomial fit. The corresponding polynomial coefficients are used in custom written software to convert the desired wavelength to the RF frequency that has to be applied to the crystal.

To focus the excitation light into a diffraction-limited spot, the light from the AOTF's exit must be coupled into a single-mode fiber to spatially filter the excitation light and provide a Gaussian illumination profile from a point source at the other end of the fiber. The fiber incoupling is done using two mirrors, incoupling optics (Thorlabs A240TM-A) and a precision XYZ stage (Thorlabs MBT613D/M). The best confocal illumination spot is achieved by projecting a small point source (singlemode fiber exit) into a small diffraction-limited illumination focus spot at the sample plane. Before the light enters the infinity-corrected imaging microscope objective, the light should be collimated and its beam diameter should roughly match the back aperture of the imaging objective. An infinity-corrected collimation objective (Thorlabs RMS4X) is used to collimate the light coming out of the single-mode fiber (NA 0.1), where a $500 \mathrm{~mm}$ telescope is used to accurately collimate the laser light (Möller-Wedel FR500/65/14.7). The collimated light from the collimation objective slightly overfills the back aperture of the imaging objective, which produces the sharpest confocal illumination spot. The collimated light is aligned to the optical axis of the microscope objective using two gimbal-mounted mirrors and two aligned diaphragms at the mounting thread of the microscope objective.

\subsubsection{Confocal detectors}

The confocal detectors act as point detectors, which measure fluorescence emission from a well-defined volume. To constrain the detection to this volume, it is necessary to remove as much stray light as possible. The main sources of stray light are leakage of excitation light through emission filters or simply stray light from reflections in the environment. Leaking excitation light can be suppressed by choosing the proper filter set with stringent bandpass characteristics. Further, appropriate measures are needed to shield the detectors and emission beam path from environmental stray light. After proper shielding, the dark noise level should match the specified level given by the manufacturer of the detector. Since in our case the confocal detection volume is static, the sample has to be raster-scanned through this detection volume to record intensity and lifetime (avalanche photo-diode) and emission spectra (spectrograph) at each point, to develop a spatially-resolved map of the appropriate observable. The incorporation of the scanning stage into the confocal detection scheme is explained at the end of this section. 


\subsubsection{Avalanche photo-diode}

The single photon counting avalanche photo-diode (APD) from Micro-PhotonDevices, Bolzano, Italy is capable of counting photons one by one and allows for accurate time-resolved lifetime and intensity analyses of the fluorophores. The avalanche-photo-diode gives a pulse when a photon is registered, which is subsequently timed by a TCSPC (time-correlated single photon counting, in our case Becker \& Hickl SPC-830) card. This TCSPC card resolves absolute photon arrival times ('macrotimes') and arrival times relative to the excitation pulse ('microtimes') from the APD's signal. The TCSPC instrumentation uses a 12 bit time-to-amplitude (4096 channel TAC) range to measure the photon arrival time with respect to the excitation pulse, which is called the microtime. This microtime is determined with a time resolution of $12 \mathrm{ps}$ in case of our 50ns TAC range. Furthermore, a macrotime counter keeps track of the TAC windows in which a photon was detected. The macrotimes are counted from the start of the recording and are afterwards converted to real-world time via multiplication by 50ns (macrotime clock). The macrotimes and microtimes together allow photon-by-photon tracking up to a time resolution of 12ps over the full recording period.

To coarse align the APD, we mounted the APD on a XYZ stage and used a $50 \mathrm{~mm}$ achromat lens to focus reflected laser light onto the APD's chip. The APD is aligned such to maximize intensity of the reflected light at the detector. Next, we optimize the alignment of the APD by maximizing the fluorescence intensity of a thin film of an efficient fluorophore (e.g. Rhodamine 6G) that was spin-coated onto a glass coverslip and mounted at the sample plane. Depending on the quality of the alignment and stray light shielding, this configuration is capable of detecting emission of single fluorophores.

\subsubsection{Spectrograph}

The spectrograph records the emission spectrum of the light coming from the confocal detection volume. The emission light that originates from the confocal detection volume is coupled into a multi-mode fiber that leads to the spectrograph. The light at the exit of the multi-mode fiber acts as a point source, which is angularly dispersed via a dispersive element (prism, grating or acousto-optical tunable filter) and projected onto a camera (typically rectangular EMCCD cameras, which have single photon sensitivity) to record the emission spectra with a high spectral resolution of approximately $1 \mathrm{~nm}$. The image at the camera is built up from multiple images of the point source (fiber exit) at different wavelengths and typically shows a rainbow colored line for a white light point source. Either a grating or a prism 


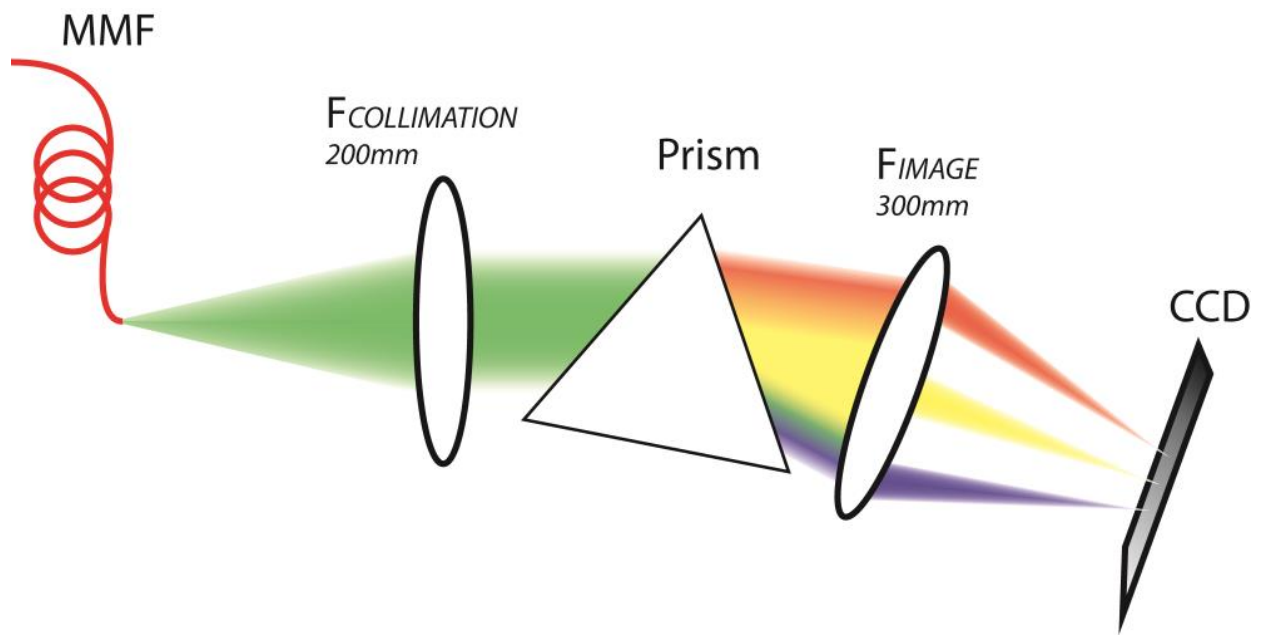

Figure 2.3 Schematic diagram of a prism spectrograph. Emission from the confocal detection volume is coupled into the multi-mode fiber (MMF) and the fiber exit acts as a point source. The light of this point source is collimated by the first lens. The prism angularly disperses the collimated beam and the imaging lens images the point source onto the CCD. The angular dispersion by the prism causes a lateral displacement of the point source image at the $\mathrm{CCD}$, which is wavelength dependent.

spectrograph can be used for multimodal imaging spectroscopy, although we used a prism spectrograph because its losses are less than $20 \%$, and we obtain the spectrum in one snapshot rather than scanning the wavelengths, which is both faster and more sensitive compared to the grating spectrograph.

A schematic diagram of a prism spectrograph is shown in Figure 2.3. For easy alignment, it is recommended to mount a tilt-adjustable $\varnothing 2 "$ mirror onto a linear stage in between the imaging lens and the camera. This allows for precise focus adjustments of the spectral image at the CCD plane, while the camera is fixed.

When the spectrograph alignment has been accomplished, the CCD needs a calibration to relate the pixel axis of the CCD to wavelength. A calibration source (HL-2000-CAL) provides distinct spectral lines that are easily identified by comparing the recorded spectrum at the $\mathrm{CCD}$ with the emission spectrum of the calibration source. A cubic polynomial fit is used to calibrate the spectrograph CCD.

\subsubsection{Scanning, synchronization scheme and cabling}

In the confocal configuration, it is possible to monitor emission from a well-defined fixed volume with high accuracy and high detection sensitivity. However, it is not possible to laterally displace this detection volume. Therefore, a piezo scanning stage is needed to scan the sample through the detection volume, with a precision of $2 \mathrm{~nm}$ and a full-range repeatability of $10 \mathrm{~nm}$, which is especially important for studying 
single emitters, since these have to be revisited after a quick localization scan. For each step, fluorescence emission is recorded using one of the confocal detectors. Accurate timing of this detection and synchronization with scanning is therefore a key aspect for confocal scanning microscopes. The best way to synchronize scanning and detection is communicating through hardware triggering. The scanning stage controller (PI E-710) serves as the master controller and triggers the detectors to record data, using TTL pulse trigger lines. Four scanning triggers can be accessed at the 'digital-IO' port at the back of the scanning stage controller. The first three trigger lines are the 'pixel', 'line' and 'frame' triggers and the fourth trigger line indicates when the 'illumination' should be active and can be used to control the activity of the excitation source. The recording time of the detectors is set beforehand. The four scanning triggers are connected directly to the TCSPC card and the 'pixel' trigger line is split to trigger the EMCCD camera that is used for the spectrograph. The 'illumination' line is also split and is used to switch the laser source on and off while scanning.

\subsection{Sample preparation}

There are many different ways to prepare a sample depending on the size, transparency, brightness of the fluorophores etcetera. One much used sample preparation method to study the photo-physical properties of single emitters is to embed the emitters in a thin polymer film to spatially separate and localize the emitters. To prepare such a sample, very clean coverslips are needed to avoid contaminating impurities. A good way to clean coverslips is by placing them in an ozone cleaner (UV/Ozone ProCleaner Plus, Bioforce, San Diego, CA) for at least one hour.

A highly diluted solution ( 1nM) of fluorophores in $2 \%$ wt polyvinyl alcohol (PVA) dissolved in spectroscopically clean water is spin-coated onto the clean coverslip at 6000RPM for 30 seconds, to embed and immobilize the single emitters in a thin film of PVA.

\subsection{Measurement procedure}

The sample is mounted into the sample plane of the microscope and the scan settings of the piezo scanning stage are defined and synchronously applied to the TCSPC hardware or Andor Newton spectrograph camera. The TCSPC hardware can be either configured to store recorded data in its RAM memory (memory configuration required) or configured to record first-in-first-out (FIFO) data, which is read out for each pixel. Emission spectra are recorded subsequently using the same 


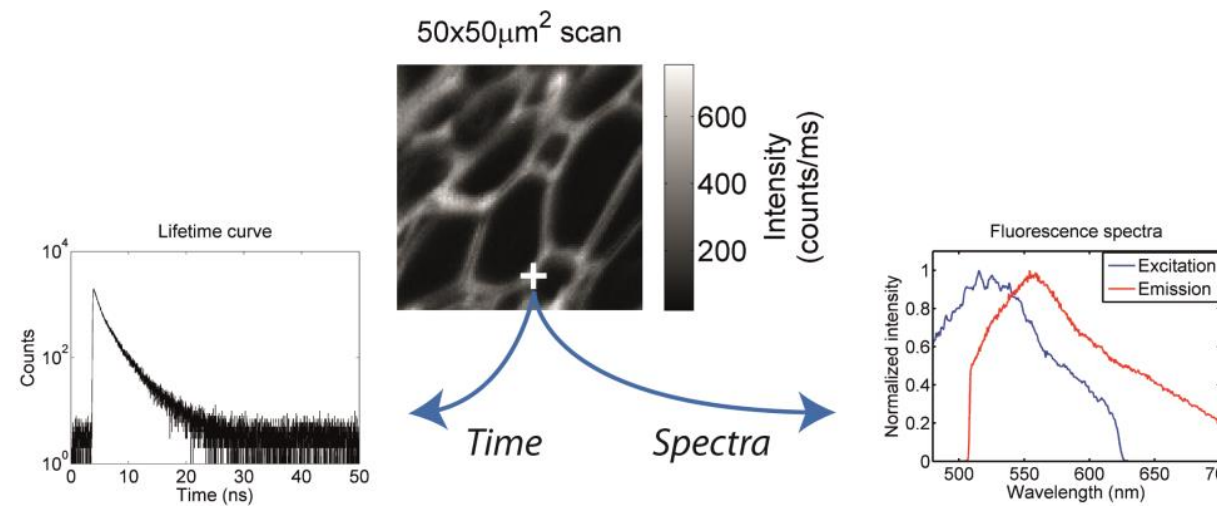

Figure 2.4 Recorded lifetime and spectral data of Convallaria Majalis at specific locations of the intensity map. First the intensity map is measured by raster scanning an area. Second, decay curves and excitation and emission spectra are recorded at a specified location, indicated by the cross. The sharp drops in the spectra indicate the cut-off edges of the filters. The nonmonoexponential decay characteristics and the broad shape of the spectra indicate that a range of fluorescent emitters is present at the specified location.

scan settings, but at a fixed excitation wavelength. For spectral imaging, the Andor Newton camera is set to 'kinetic' readout mode with 'fast external' triggering. The kinetic series length equals the total number of pixels that will be scanned during the measurement. After arming the confocal detector(s), the detectors will wait for scantriggers from the scanning stage controller and will start recording when the scanning stage controller starts moving the stage from pixel to pixel.

The multimodal microscope can record intensity, lifetime, excitation spectra and emission spectra in the configuration described in this chapter. From a practical perspective, it is recommended to fix the excitation wavelength and first record an intensity map to find the right orientation of the sample (confocal microscopy) and secondly record a lifetime map and spectral maps (Figure 2.4). Here, it is very important to choose appropriate short-pass filters and long-pass filters as described in the section 'confocal detectors'. For both excitation and emission spectra it is very important to measure background spectra as well, since these are required for proper data analysis.

\subsection{Analysis}

The 'measurement procedure' describes how to record intensity, lifetime, excitation and emission spectra for each pixel. Once these data has been acquired, there are a few post-processing steps required to correct this data, depending on the data type. These corrections will be described for each observable in separate sections below. 
After applying the appropriate corrections, the temporal and spectral properties of each pixel are known.

\subsubsection{Intensity and lifetime}

The intensity is calculated from the number of photons of the recorded FIFO photon stream'. In case that the background (number of uncorrelated photons) is high compared to the fluorescence signal, one can use the decay curve to filter out uncorrelated background photons. A threshold can be used that defines occurrences in the decay curve that correspond to fluorescence photons, which are then separated from uncorrelated photons. The number of fluorescence photons is then used to calculate the intensity map.

The 'SPC Image' software package of Becker and Hickl GmbH allows for quantitative lifetime analyses of the fluorescence decay curves that are measured at each pixel. The measured decay curves are typically fitted with a mono-exponential decay model, but can be fitted with a multi-exponential decay model if the decay characteristic is complex. The lifetime, which is the time-constant of the spontaneous emission process, is calculated for each pixel to construct a lifetime map.

\subsubsection{Emission spectrum}

The emission spectrum is recorded with the Andor Newton EMCCD camera, which is attached to the prism spectrograph. This recorded spectrum $(S(\lambda))$ needs to be background $(B(\lambda))$ subtracted and corrected for spectral response of the detection system $(R(\lambda))$ (equation 2.1). The spectral response correction is not required if the spectral response of the detection system is smooth and has only minor changes over the spectral range of the recorded emission spectrum.

$$
S_{e m}(\lambda)=\frac{S(\lambda)-B(\lambda)}{R(\lambda)}
$$

\subsubsection{Excitation spectrum}

The excitation spectrum resembles the absorbance spectrum in case the quantum efficiency remains constant for all excitation wavelengths. Proper corrections should

\footnotetext{
The photon streams are recorded with the APD and TCSPC card for each pixel. For each photon both 'macrotime' and 'microtime' information was recorded, as explained in section 2.4.2.1. Macrotimes are used to construct an intensity trace and microtimes are used for decay dynamics, which is generally calculated by making a histogram of the 'microtimes' of all of the recorded photons. As with the intensity trace, 'microtimes' can also be used to construct decay traces, by calculating the average photon arrival time per unit time.
} 
involve background subtraction and wavelength dependent photon flux corrections ${ }^{2}$, as indicated by equation 2.2 .

$$
S_{\text {exc }}(\lambda)=\frac{S(\lambda)-B(\lambda)}{\Phi_{d}(\lambda)}
$$

where $S_{\text {exc }}$ is the corrected excitation spectrum that is calculated from the measured fluorescence intensity at different wavelengths $S(\lambda)$, background spectrum $B(\lambda)$ and photon flux density $\Phi_{d}(\lambda)$ of the excitation light. The photon flux density is the number of photons per second $(\Phi(\lambda))$ per unit area, where the diffraction limited area $A(\lambda)$ scales with $\lambda^{2}$ (equation 2.3). The photon flux $(\Phi(\lambda)$ ) is determined from the illumination power $P(\lambda)$ divided by the energy of a single photon $E_{p h}(\lambda)$, which inversely scales with $\lambda$.

$$
\Phi_{d}(\lambda)=\frac{\Phi(\lambda)}{A(\lambda)}=\frac{P(\lambda)}{E_{p h}(\lambda) \cdot A(\lambda)} \sim \frac{P(\lambda)}{\lambda}
$$

\subsection{Excitation spectral imaging}

Excitation spectral imaging allows one to investigate the local fluorescence absorbance properties of a sample by recording an excitation spectrum for each pixel. Convallaria Majalis is used as a test sample and an excitation spectral data cube was acquired by raster scanning the same area at 30 different excitation wavelengths ranging from $450 \mathrm{~nm}$ to $600 \mathrm{~nm}$ (Figure 2.5a). The scanned area is $100 \times 100$ pixels in size and fluorescence emission was collected within $2 \mathrm{~ms}$ for each pixel. The spectral data cube shows that at blue excitation the fluorescence emission at the cell crossings is high and that the emission at the cell wall interfaces is comparatively lower. The intensity of the spectral data cube can be translated into a false color image (Figure $2.5 \mathrm{~b})$ by specifying bands of interest, e.g. 560-600nm (R), 530-550nm (G), 485$500 \mathrm{~nm}(\mathrm{~B})$ in this example. The intensities within these bands are converted into a 2D false color RGB map to give an impression of the differences in spectral absorption between the lateral coordinates of the 3D spectral data cube. We see that the cell crossings appear blue and that the cell wall interfaces are slightly greener, indicating small spectral variation along the cell wall.

\footnotetext{
${ }^{2}$ For single emitters, the fluorescence emission scales with the excitation photon flux density, which inversely scales with the area of the diffraction limited spot. However, the fluorescence emission of an ensemble scales with excitation photon flux only, because the number of illuminated emitters linearly scales with the illumination volume, which one-to-one compensates the inversely scaling flux density $(\Phi(\lambda) \sim P(\lambda) \cdot \lambda)$.
} 


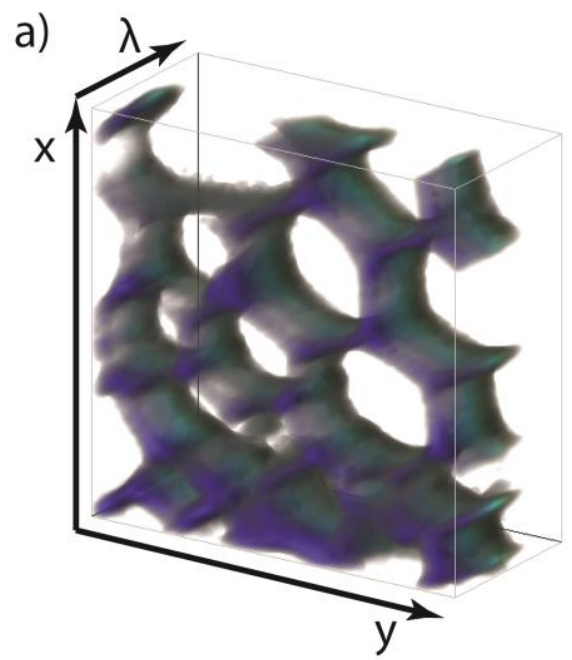

b)

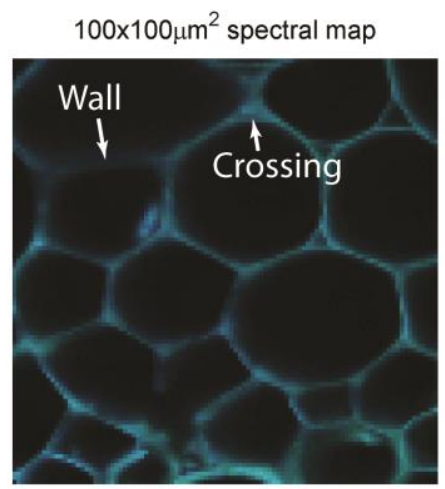

c)

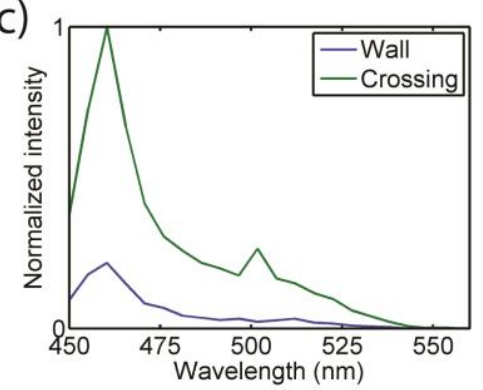

Figure 2.5 Excitation spectral data cube (a) of a $100 \times 100 \mu \mathrm{m}^{2}$ area of Convallaria Majalis. This data cube contains spectral information for each lateral coordinate in the scanning area. The excitation intensity at blue wavelengths is lower at the cell wall interface compared to the cell crossing, reflecting a difference in absorption at these wavelengths. The fluorescence emission at different bands can be combined into a single 2D false color map (b) to provide an overview of the differences in spectral absorbance across the image. One can use this $2 \mathrm{D}$ false color map to locate and select points of interest and analyze or compare the local spectral information (c).

One can use this 2D false color map to locate and select points of interest to analyze the local spectral information (Figure 2.5c). By comparing the excitation spectra of points at the cell crossing and cell wall interface, we see that the relative absorption of blue light for the cell wall interface is less compared to the cell crossing, which explains the slightly more green color in the false color map. Furthermore, we observe an overall lower light absorption for the cell wall interface. Since the shapes of the two spectra are alike, it is likely that the concentration of fluorophores is lower at the cell wall interface, giving a reduced fluorescence emission. 
Combining multimodal microscopy with excitation spectral imaging gives access to hyperspectral FLIM [19-21], which opens up possibilities for more sophisticated analyses like spectral cross-correlation that is often used in hyperspectral imaging to spectrally unmix the spectral data cubes and identify characteristic features at an image.

\subsection{Single emitters}

The preceding examples described how the multimodality of the setup is utilized to study time-resolved and spectrally resolved characteristics at specified locations within a scanned area. For these ensemble studies, the measured characteristics reflect the average properties of the ensemble of fluorophores within that spot, meaning that the individual characteristics of single emitters are obscured by ensemble averaging. To access the individual properties of the single emitters, a highly diluted fluorophore sample is prepared to spatially separate and isolate the single emitters (see section 2.5). Single emitter samples are raster-scanned to localize the single emitters, typically looking like the intensity area scans shown in Figure 2.6.

The single emitters appear as round dots in Figure 2.6, which are clearly distinguishable from the background. Note that the size of the round dots does not reflect the actual size of the single emitters, but is related to the width of the diffraction limited excitation spot, which is convoluted with the sample, causing a convoluted intensity profile around each single emitter. Occasionally, dark pixels are observed at the location of the single emitters, which are directly linked to a characteristic feature of single emitters, called blinking. Fluorescence emission intermittency, or blinking, is the sudden switching of a fluorophore from one state to another, which is often paired with a change in emission intensity (see Figure 2.7). The nature of blinking depends on the fluorophore and is still an actively debated topic. Blinking of quantum dots will be studied in detail later in this thesis.

After locating the single emitters in an emission intensity map, one can revisit these locations and probe the characteristics of the single emitters (point-andprobe). A number of parameters that characterize single emitter emission are now routinely accessible at ambient temperatures, including emission intensity and polarization [22, 23], fluorescence lifetime [24-26], and the emission spectrum [2729]. Access to these parameters yields unique insights into distinct properties of single emitters, and enables the determination of the distributions of the relevant experimental parameters, revealing, for example, distinct substates and energetic levels in a heterogeneous population [30, 31]. 
a)

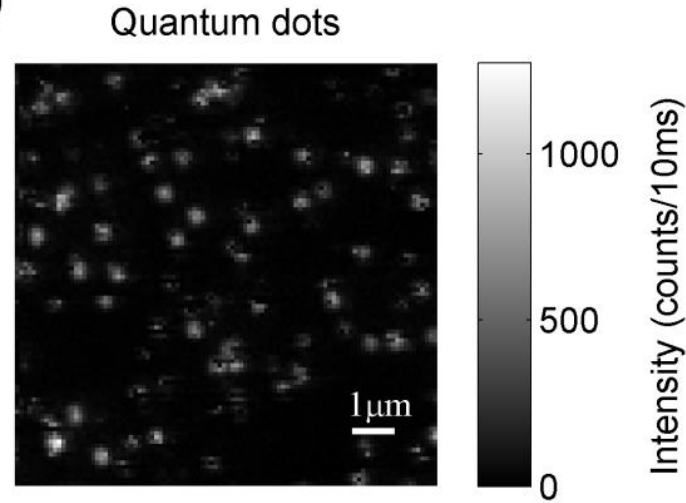

b)

Perylene dyes
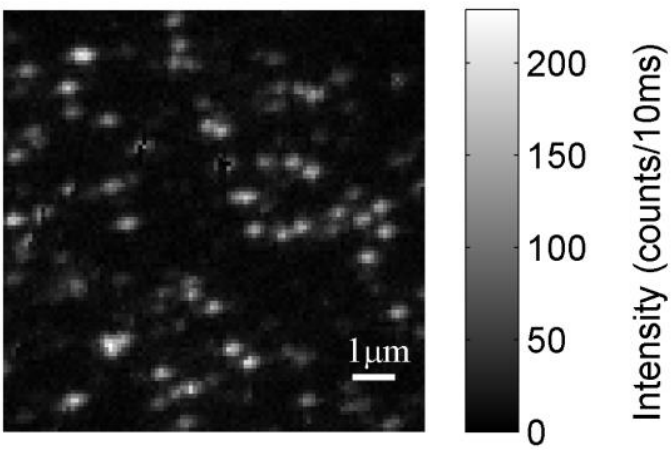

Figure 2.6 Raster-scanned 10x10 $\mathrm{mm}^{2}$ intensity images of single semiconductor nanocrystals (quantum dots) (a) and single organic dyes (perylene) (b)

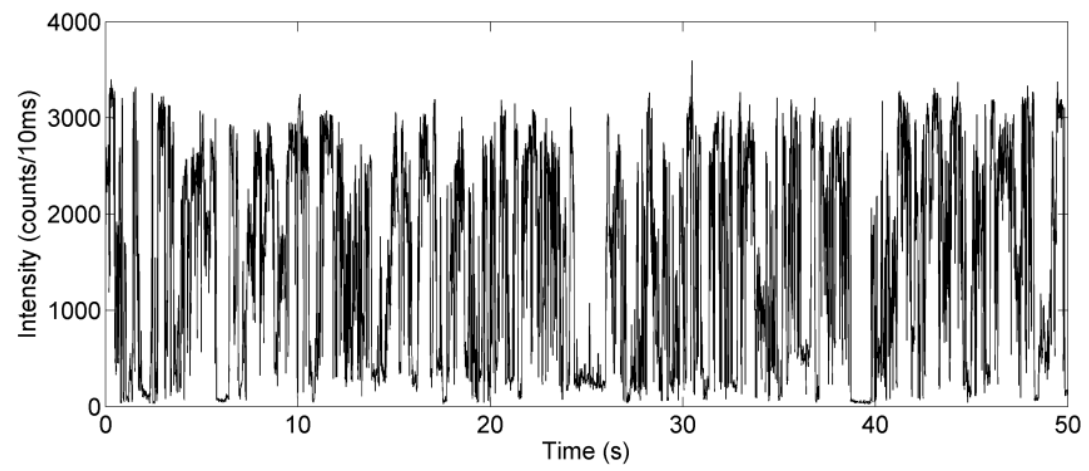

Figure 2.7 Fluorescence emission intensity trace of a single quantum dot, clearly showing blinking 
In the next two chapters we extend the range of observable experimental parameters at the single emitter level, by demonstrating measurements of the spectral absorbance properties and the Stokes shift of single emitters.

\section{REFERENCES}

1. Blum, C., et al., Multimode microscopy: Spectral and lifetime imaging. Journal of The Royal Society Interface, 2009. 6(Suppl 1): p. S35-S43.

2. Bolinger, J.C., et al., Conformation and energy transfer in single conjugated polymers. Acc. Chem. Res., 2012. 45(11): p. 1992-2001.

3. Borsch, M. and J. Wrachtrup, Improving fret-based monitoring of single chemomechanical rotary motors at work. Chemphyschem, 2011. 12(3): p. 542-553.

4. Buning, R. and J. van Noort, Single-pair fret experiments on nucleosome conformational dynamics. Biochimie, 2010. 92(12): p. 1729-1740.

5. Schierle, G.S.K., et al., A fret sensor for non-invasive imaging of amyloid formation in vivo. Chemphyschem, 2011. 12(3): p. 673-680.

6. Kuhn, S., et al., Enhancement of single-molecule fluorescence using a gold nanoparticle as an optical nanoantenna. Physical Review Letters, 2006. 97(1).

7. Schlegel, G., et al., Fluorescence decay time of single semiconductor nanocrystals. Phys Rev Lett, 2002. 88(13): p. 137401.

8. Chan, F.T.S., C.F. Kaminski, and G.S.K. Schierle, Homofret fluorescence anisotropy imaging as a tool to study molecular self-assembly in live cells. Chemphyschem, 2011. 12(3): p. 500-509.

9. $\quad$ Early, K.T., et al., Linear dipole behavior in single cdse-oligo(phenylene vinylene) nanostructures. Acs Nano, 2009. 3(2): p. 453-461.

10. Taminiau, T.H., et al., Optical antennas direct single-molecule emission. Nat Photon, 2008. 2(4): p. 234-237.

11. Fereidouni, F., A.N. Bader, and H.C. Gerritsen, Spectral phasor analysis allows rapid and reliable unmixing of fluorescence microscopy spectral images. Optics Express, 2012. 20(12): p. 12729-12741.

12. Hildner, R., et al., Single-molecule spectroscopy on a ladder-type conjugated polymer: Electron-phonon coupling and spectral diffusion. Chemphyschem, 2009. 10(14): p. 2524-2534.

13. Kang, G.P., et al., Forster resonance energy transfer as a probe of membrane protein folding. Biochimica Et Biophysica Acta-Biomembranes, 2012. 1818(2): p. 154-161.

14. Betz, T., et al., Excitation beyond the monochromatic laser limit: Simultaneous 3-d confocal and multiphoton microscopy with a tapered fiber as white-light laser source. Journal of Biomedical Optics, 2005. 10(5): p. 054009-054009.

15. Frank, J.H., et al., A white light confocal microscope for spectrally resolved multidimensional imaging. Journal of Microscopy, 2007. 227(3): p. 203-215.

16. Owen, D.M., et al., Excitation-resolved hyperspectral fluorescence lifetime imaging using a uv-extended supercontinuum source. Opt. Lett., 2007. 32(23): p. 3408-3410.

17. Blum, C., et al., Room temperature excitation spectroscopy of single quantum dots. Beilstein Journal of Nanotechnology, 2011. 2: p. 516-524. 
18. Engelborghs, Y. and A.W.J.G. Visser, Fluorescence spectroscopy and microscopy methods and protocols. Methods in molecular biology, ed. J.M. Walker. 2014: Springer Protocols. 816.

19. Nie, Z.J., et al., Hyperspectral fluorescence lifetime imaging for optical biopsy. Journal of Biomedical Optics, 2013. 18(9): p. 7.

20. De Beule, P., et al., Rapid hyperspectral fluorescence lifetime imaging. Microscopy Research and Technique, 2007. 70(5): p. 481-484.

21. Gutierrez-Navarro, O., et al., A fully constrained optimization method for timeresolved multispectral fluorescence lifetime imaging microscopy data unmixing. Ieee Transactions on Biomedical Engineering, 2013. 60(6): p. 1711-1720.

22. Forkey, J.N., M.E. Quinlan, and Y.E. Goldman, Protein structural dynamics by single-molecule fluorescence polarization. Progress in Biophysics and Molecular Biology, 2000. 74(1-2): p. 1-35.

23. Peterman, E.J.G., S. Brasselet, and W.E. Moerner, The fluorescence dynamics of single molecules of green fluorescent protein. The Journal of Physical Chemistry A, 1999. 103(49): p. 10553-10560.

24. Kapusta, P., et al., Fluorescence lifetime correlation spectroscopy. Journal of Fluorescence, 2007. 17(1): p. 43-48.

25. Schleifenbaum, F., et al., New insights into the photophysics of dsred by multiparameter spectroscopy on single proteins. The Journal of Physical Chemistry B, 2008. 112(25): p. 7669-7674.

26. Tinnefeld, P., et al., Confocal fluorescence lifetime imaging microscopy (flim) at the single molecule level. Single Molecules, 2000. 1(3): p. 215-223.

27. Blum, C., A.J. Meixner, and V. Subramaniam, Spectral versatility of single reef coral fluorescent proteins detected by spectrally-resolved single molecule spectroscopy. Chemphyschem, 2008. 9(2): p. 310-315.

28. Cotlet, M., et al., Identification of different emitting species in the red fluorescent protein dsred by means of ensemble and single-molecule spectroscopy. Proceedings of the National Academy of Sciences, 2001. 98(25): p. 14398-14403.

29. Lacoste, T.D., et al., Ultrahigh-resolution multicolor colocalization of single fluorescent probes. Proceedings of the National Academy of Sciences, 2000. 97(17): p. 9461-9466.

30. Blum, C., A.J. Meixner, and V. Subramaniam, Room temperature spectrally resolved single-molecule spectroscopy reveals new spectral forms and photophysical versatility of aequorea green fluorescent protein variants. Biophysical Journal, 2004. 87(6): p. 4172-4179.

31. Widengren, J., et al., Single-molecule detection and identification of multiple $s$ pecies by multiparameter fluorescence detection. Analytical Chemistry, 2006. 78(6): p. 2039-2050. 



\title{
Chapter 3
}

\section{Room temperature excitation spectroscopy of single quantum dots}

\begin{abstract}
Although many photophysical observables are accessible at the single emitter level, a detailed study of frequency resolved excitation dependent processes at the single emitter level at room temperature has not experimentally been achievable so far. This chapter describes the results of a pilot study in which we tested a single emitter detection scheme to investigate excitation spectra of single emitters at room temperature. We used single CdSe nanocrystals (quantum dots) as fluorescent probes, which have broad band spectral absorption properties, and demonstrate the potential of single emitter photoluminescence excitation spectroscopy by recording excitation spectra over a wide spectral range of $100 \mathrm{~nm}$. The spectra exhibit emission intermittency, which is a characteristic feature of single emitters. We observe large variations in the spectra close to the band edge, representing the individual heterogeneity of the observed quantum dots. We expect that the additional capability of recording excitation spectra at room temperature from single emitters will enable insights into the photophysics of emitters that so far have remained inaccessible.
\end{abstract}

This chapter has been published in the Beilstein Journal of Nanotechnology, 2011, 2, pages 516-524, Room temperature excitation spectroscopy of single quantum dots, doi: 10.3762/bjnano.2.56 


\subsection{Probing spectral absorbance of single emitters}

The primary challenge has been the intrinsic difficulty in measuring the absorption of a single emitter at room temperature due to the extremely low signal to noise ratio. Although recent reports have demonstrated the detection of single emitter absorbance [1-4], a complete single emitter absorbance spectrum at room temperature has not yet been reported. A complementary approach to access the frequency dependent coupling of an emitter to an external electromagnetic field can be based on photoluminescence excitation spectroscopy. The excitation spectrum, commonly used in ensemble fluorescence spectroscopy, depicts the evolution of the emission intensity recorded in a fixed spectral detection window upon scanning the excitation wavelength. Moreover, the excitation spectrum of an emitter coincides with its absorbance spectrum if the quantum efficiency is independent of excitation wavelength, which is generally assumed to be true for most emitters over large wavelength ranges. Single emitter photoluminescence excitation microscopy has been already achieved in the early days of single emitter detection, but has been limited to experiments at cryogenic temperatures where the linewidths of individual emitters are not inhomogeneously broadened [5, 6]. Hence, only a very limited excitation wavelength range was required to resolve individual absorbance properties of a single emitter at low temperatures. Broadband excitation spectroscopy is hard to achieve at the single emitter level, because it requires a light source that has both high brightness and wide spectral tunability. A laser is often required for single emitter experiments to provide the necessary power, but is a mono-chromatic light source and cannot provide the required wide spectral tunability. However, super-continuum laser sources have recently become commercially available, and provide both high power and wide spectral tunability (see previous chapter), allowing for broadband excitation spectroscopy of single emitters at ambient conditions.

In this chapter, the first successful acquisition of single emitter excitation spectra at ambient conditions over a wide spectral range is described. We combine both a tunable white-light laser source and a confocal microscope with single emitter detection sensitivity to demonstrate the spectrally-resolved absorbance properties of isolated semiconductor nanocrystals (quantum dots) by measuring the excitation spectra of these single emitters. We demonstrate here how single emitter excitation spectroscopy provides an addition to the range of single emitter spectroscopy techniques, yielding new insights into the complex photophysics of quantum dots. 


\subsection{Setup and measurement procedure}

The two essential requirements to realize room temperature single emitter excitation microscopy are an excitation source that delivers high power, collimated and monochromatic excitation light over a broad wavelength range $(>100 \mathrm{~nm})$, and the ability to locate and analyze the single emitters. Lasers are typically implemented to deliver a high power at the single emitter. Therefore, these requirements dictate a microscopy approach that combines a tunable white-light laser - for spectrally resolved excitation - with a single emitter sensitive confocal setup to achieve the highest possible lateral resolution and the highest sensitivity in detection (Figure 3.1).

\subsubsection{Sweeping excitation source}

We used a Fianium SC400-PP pulsed supercontinuum laser source to provide $2 \mathrm{~W}$ broad spectral excitation power ranging from $400 \mathrm{~nm}$ to $2000 \mathrm{~nm}$ at $20 \mathrm{MHz}$ repetition rate. The white light was focused on the entrance slit of an Acton SpectraPro-300i grating monochromator (300 grooves $/ \mathrm{mm}$, blazed for $500 \mathrm{~nm}$ ), and light of a narrow spectral range $(<3 \mathrm{~nm})$ was collected at the exit slit of the

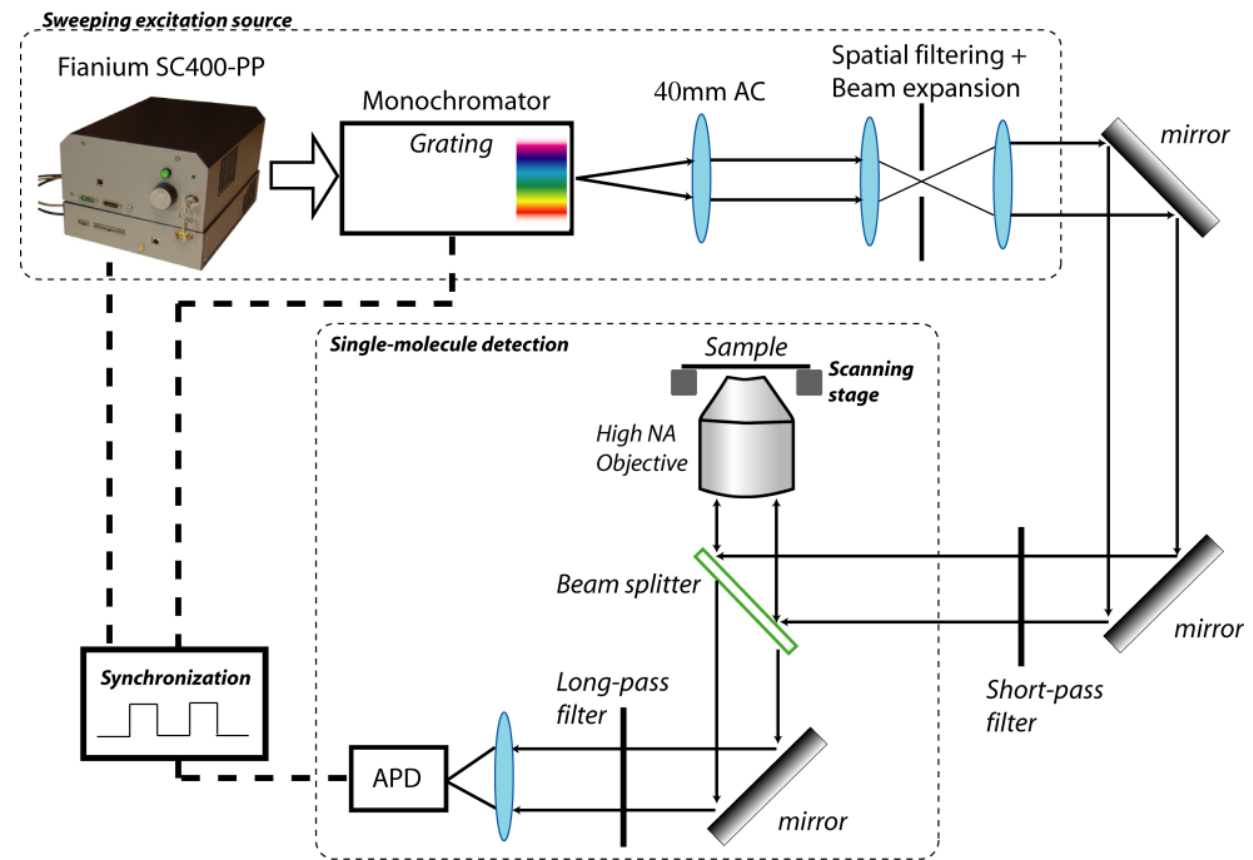

Figure 3.1 Schematic of the single emitter excitation setup. The laser beam from the sweeping excitation source has a narrow excitation band and slightly overfills the back-aperture of the imaging objective. The wavelength of the excitation source is varied across the visible spectral range and the emission intensity is monitored by the confocal detector. For each excitation wavelength, the emission intensity of the single emitter is recorded over time. 
monochromator. To increase the beam quality and to expand the beam size we used a spatial filtering system consisting of achromatic lenses and a pinhole. Wavelength selection was achieved by computer-controlled rotation of the grating inside the monochromator. In this way, excitation sweeps from $520 \mathrm{~nm}$ to $620 \mathrm{~nm}$ excitation wavelength with step size of $1 \mathrm{~nm}$ were achieved. The available excitation power increased with increasing wavelength and was corrected for in the data analysis (explained in section 3.3.1).

\subsubsection{Single emitter sensitive detection}

The single emitter sensitive detection is in essence similar to what is described in chapter 2. In addition, to effectively suppress excitation light at the detector we used a $620 \mathrm{~nm}$ short-pass filter (SP01-633RU-25, Semrock, Rochester, NY, USA) in the excitation path, and in the detection path a $625 \mathrm{~nm}$ long-pass filter (LP02-633RU-25, Semrock) in combination with a $650 \mathrm{~nm}$ band-pass filter (FF01-670/30-25, Semrock).

\subsection{Experiment}

\subsubsection{Recording single emitter excitation spectra}

The experiments were carried out in two steps. After preparing a sample with single quantum dots (eFluor650, eBioscience, UK), the single quantum dots were localized by raster-scanning an area of $20 \times 20 \mu m^{2}$ of the sample using a fixed excitation wavelength $\left(\lambda_{e x}=600 \mathrm{~nm}\right)$ and creating an emission intensity image (for example, see chapter 2, section 2.9). After the localization, the single quantum dots were positioned in the laser focus and the excitation wavelength was swept from $520 \mathrm{~nm}$ to $620 \mathrm{~nm}$ in increments of $1 \mathrm{~nm}$. At each excitation wavelength we recorded the emission intensity for $400 \mathrm{~ms}$. After this $400 \mathrm{~ms}$ recording period, the hardware was preparing for the next wavelength and the detector was disabled, giving absolute zero values in the intensity trace (see Figure 3.2, left). These flags were later used in the data analysis to synchronize excitation wavelengths and the measured time-trace offline.

Besides the flags, the time series shows some emission dips caused by blinking, which is indicative of single emitter emission. Furthermore, the envelope of the full time-series shows a peak around $600 \mathrm{~nm}$, where one would expect to find a pronounced excitonic peak of the $1 \mathrm{~S}(\mathrm{e})-2 \mathrm{~S} 3 / 2(\mathrm{~h})$ transition [7]. In a first step to construct the photoluminescence excitation spectrum, the total emission counts per 


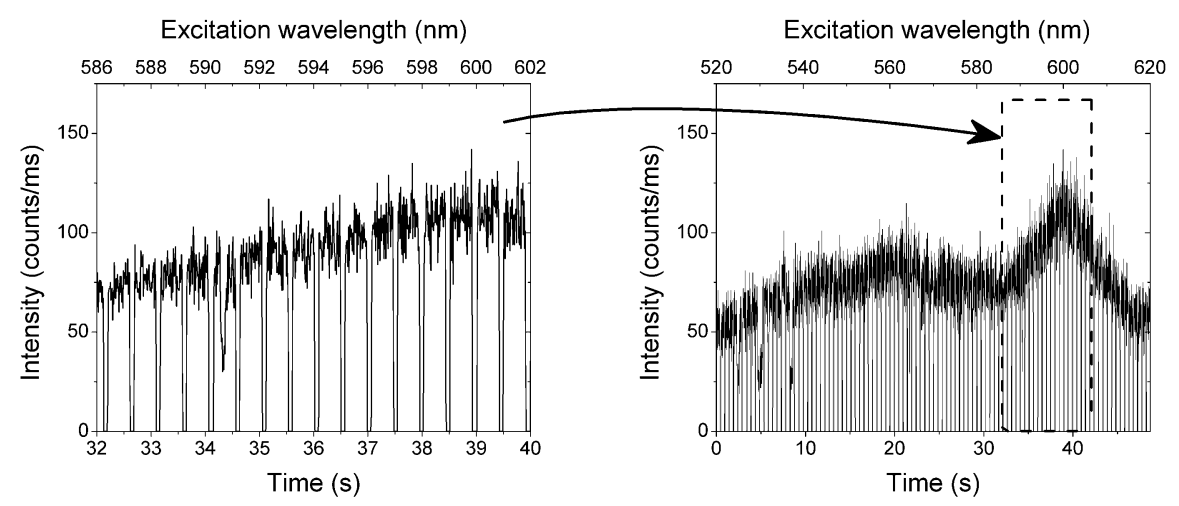

Figure 3.2 The left image shows a series of time-traces, with a bin time of $5 \mathrm{~ms}$, that were recorded for each excitation wavelength. The flags are clearly visible as absolute zero values indicating the change of excitation wavelength where the excitation source and the detector were disabled. The full series of time-traces of a single quantum dot is shown on the right, before any corrections for excitation intensity and detection efficiency were applied.
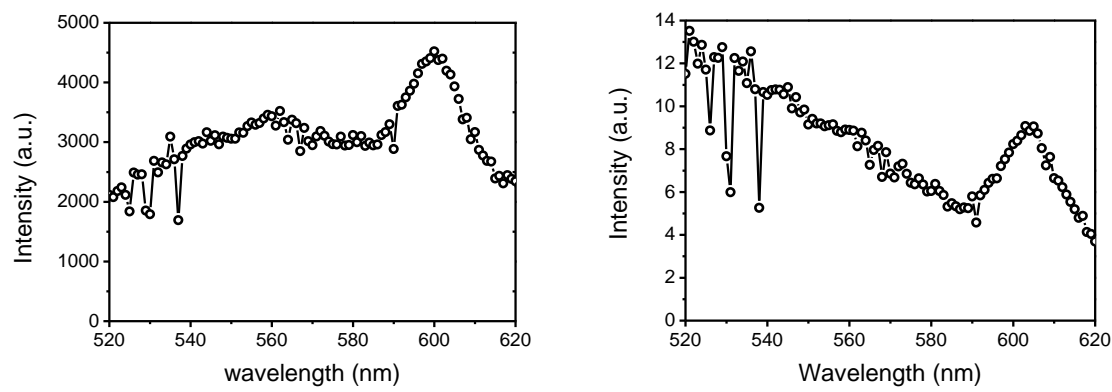

Figure 3.3 The raw single quantum dot photoluminescence excitation spectrum was calculated by integration of all photons detected per each excitation wavelength. The pronounced 1S(e)$2 \mathrm{~S} 3 / 2$ (h) transition is already clearly visible without further correction of this raw data and was found at the expected wavelength of around $600 \mathrm{~nm}$. The corrected single quantum dot excitation spectrum is shown on the right, and clearly shows a declining slope at the short wavelength side of the spectrum, which represents the quasicontinuum.

excitation wavelength were integrated, giving a single total intensity per excitation wavelength (Figure 3.3, left).

To obtain the corrected photoluminescence excitation spectrum of a single emitter from the raw data, as presented on the left in Figure 3.3, the data was background corrected and calibrated using a reference spectrum recorded for a film of the quantum dot emitters (i.e. an ensemble of emitters) to compensate for wavelength dependent excitation power and detection efficiency. The resulting corrected spectrum is shown on the right in Figure 3.3. After correction, the shape of 
the excitonic peak is similar to that of the uncorrected spectrum. Furthermore, a steady increase is observed at shorter wavelengths, which we attribute to the quasicontinuum region of the quantum dot. Compared to the uncorrected spectrum, the intensity at the quasicontinuum is higher, being caused by compensation of lower excitation power for wavelengths in the quasicontinuum range.

Quantum dots exhibit a discrete structure of quantized energy states. Hence, one would expect to observe discrete bands in the excitation spectra when the excitation wavelength is in resonance with a transition to such a state. Low temperature experiments have shown narrow emission lines [8], but also revealed that only a few sharp transitions in the direct vicinity of the band edge can be found, while at higher excitation energies the optical transitions merge into a dense quasicontinuum [9]. At room temperature, these sharp transitions experience homogeneous broadening effects, mainly due to lattice vibrations. It is therefore not surprising that at room temperature no discrete bands are observed in the excitation spectra of quantum dots.

Moreover, the excitation spectrum shows dips, originating from blinking which results in time-averaged intensity levels. A majority of the recorded excitation spectra shows emission intermittency (or blinking, see chapter 5 and 6 for more details on blinking of quantum dots), which is a clear indication for the observation of a quantum emitter. In addition to the intensity blinking observed, we confirmed that we were addressing a single emitter and thus collected single photons at a time. We analyzed the coincidence of detected photons in time using two separate detectors in a Hanbury Brown and Twiss configuration [10]. A single photon passing a 50:50 beamsplitter has an equal chance to proceed either towards the one detector or the other detector, but can only go to one of the two detectors because a single photon cannot split. Correlating photon arrival time traces from one detector with the other detector results ideally in a zero correlation of the fluorescence emission events. The resulting photon-antibunching curves recorded in this manner exhibited a near zero correlation $\left(g^{(2)}=0.3\right)$ for the detection of two photons at the same time, as shown for a typical example in Figure 3.4c. Photon-antibunching data give strong evidence for the observation of single emitters [11], since a single emitting system intrinsically cannot emit two photons at the same time. Generally, $g^{(2)}$ values below 0.5 are accepted as a proof of single emitter observation [12].

\subsubsection{Excitation spectra of single quantum dots}

In this study, we recorded excitation spectra of 48 individual quantum dots at room temperature. Similar to the single emitter excitation spectrum in Figure 3.3, the 
recorded single quantum dot excitation spectra exhibit the main characteristics of the ensemble spectrum, having a declining slope from shorter to longer wavelengths and a peak close to the band edge transition which we identify as the $1 \mathrm{~S}(\mathrm{e})-2 \mathrm{~S} 3 / 2(\mathrm{~h})$ transition. However, we find distinct differences in the individual spectra that can be attributed to individual photophysical properties of the analyzed single quantum dots (see section 3.3.3) as well as to the well-known transitions of single emitters to dark non-emitting states. A typical example of an excitation spectrum obtained from a single quantum dot is shown in Figure $3.4 \mathrm{a}$ and is compared with the excitation spectrum of the ensemble (dashed red line). In general, we observe distinct intensity dips and gaps in a majority of the single quantum dot excitation spectra, caused by blinking of the quantum dots.

The left panel of Figure $3.4 \mathrm{~b}$ depicts the intensity evolution for excitation from $522 \mathrm{~nm}-524 \mathrm{~nm}$, where the green line marks the background signal level without quantum dot emission, attributed to the emitter being in a dark state. For these wavelengths, the excitation spectrum shows strong intensity fluctuations. At $\lambda_{e x}=522 \mathrm{~nm}$, an intensity jump is observed immediately before the subsequent

a)

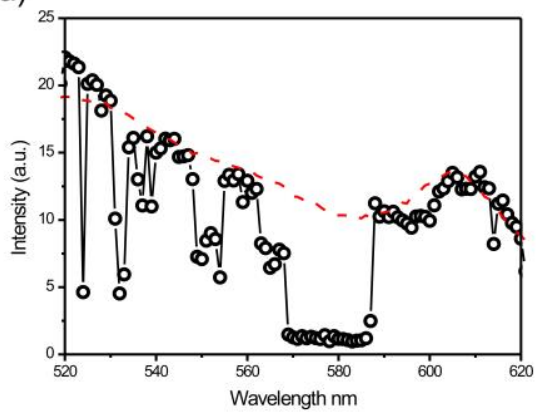

c)

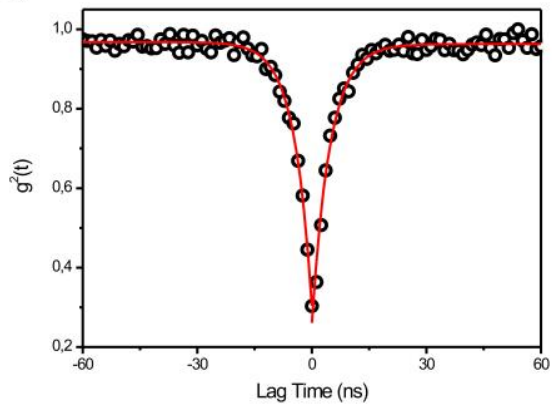

b)
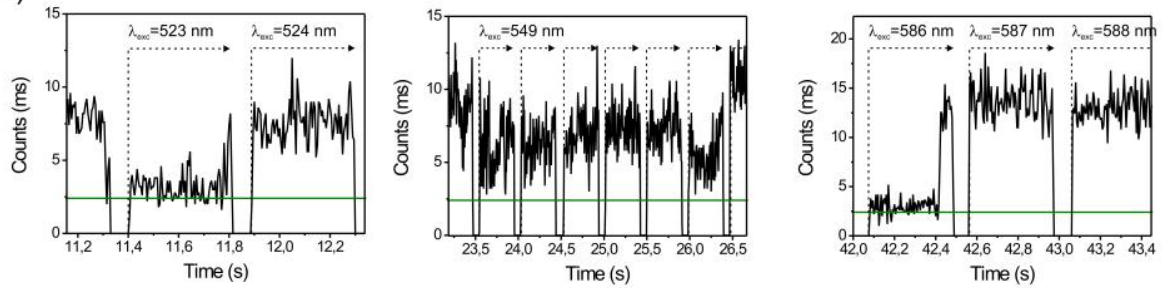

Figure 3.4 Single emitter characteristics observed by excitation spectroscopy of isolated quantum dots. a) Excitation spectrum of a single quantum dot (open circles) with typical intensity intermittencies that result in drops and gaps in the spectrum. The ensemble excitation spectrum is shown for comparison (red dashed line). b) Intensity trajectories of a single quantum dot for selected excitation wavelengths. c) Photon antibunching curve of a single quantum dot. 
wavelength change. For $\lambda_{\text {ex }}=523 \mathrm{~nm}$, the quantum dot is still in a non-emitting offstate, indicated by the signal intensity at background levels. Just before $11.8 \mathrm{~s}$ it returns into a stable on-state after some initial short "bursts". Accordingly, the coexistence of both the emitting and non-emitting states in the intensity trace results in time-averaged intensities in the excitation spectrum, and the intensity in the excitation spectrum does not always drop to the background level, as the quantum dot is not necessarily dark during the whole integration interval.

The middle panel in Figure 3.4b depicts decreased emission intensity that varies over time but does not drop to the background level. These variations in the observed emission intensity are explained either as the result of fast blinking below the time resolution of the experiment or by transitions of the quantum dot to a dim (weakly-emitting) state [13]. As a result of this reduced emission we observe drops in the excitation spectrum over a number of wavelengths as can be seen around $550 \mathrm{~nm}$ in Figure 3.4a. Finally, we regularly find extended gaps in the excitation spectrum of single quantum dots as can be seen in the spectrum between $569 \mathrm{~nm}$ and $587 \mathrm{~nm}$ (Figure 3.4a). This gap in the excitation spectrum is attributed to a long lasting dark state followed by the return to an emitting state as can be seen in the right panel of Figure 3.4b.

It is important to note that the observed drops in intensity do not correlate with changes in the excitation wavelength. Clearly, the observed drops and gaps in the spectrum would vanish if exclusively emitting states were observed for each excitation wavelength. For detection intervals where a transition to or from a dark state occurs (e.g. see Figure 3.4b, right panel), this correction can easily be performed by just considering the higher emission intensity level. Drops and gaps in the spectrum originating from dim states or very fast transitions below the sampling resolution and from long-lasting dark states longer than the integration time per excitation wavelength, can be avoided by repeated, possibly faster, scanning of the excitation wavelength that requires further technical development for implementation in future studies.

\subsubsection{Individual spectral properties of the single emitters}

Our data also give access to the individual spectral properties of the quantum dots. In Figure 3.5 we show some typical examples of single quantum dot excitation spectra. As a guide to the eye and for comparison, the reference ensemble excitation spectrum is plotted as a dashed red line in each panel of Figure 3.5. The recorded spectra show varying degrees of blinking ranging from spectra exhibiting almost no 
dips and gaps due to emission intermittencies (Figure 3.5, top panel) to spectra where numerous transitions between emitting and dark states can be observed (Figure 3.5, bottom panel).

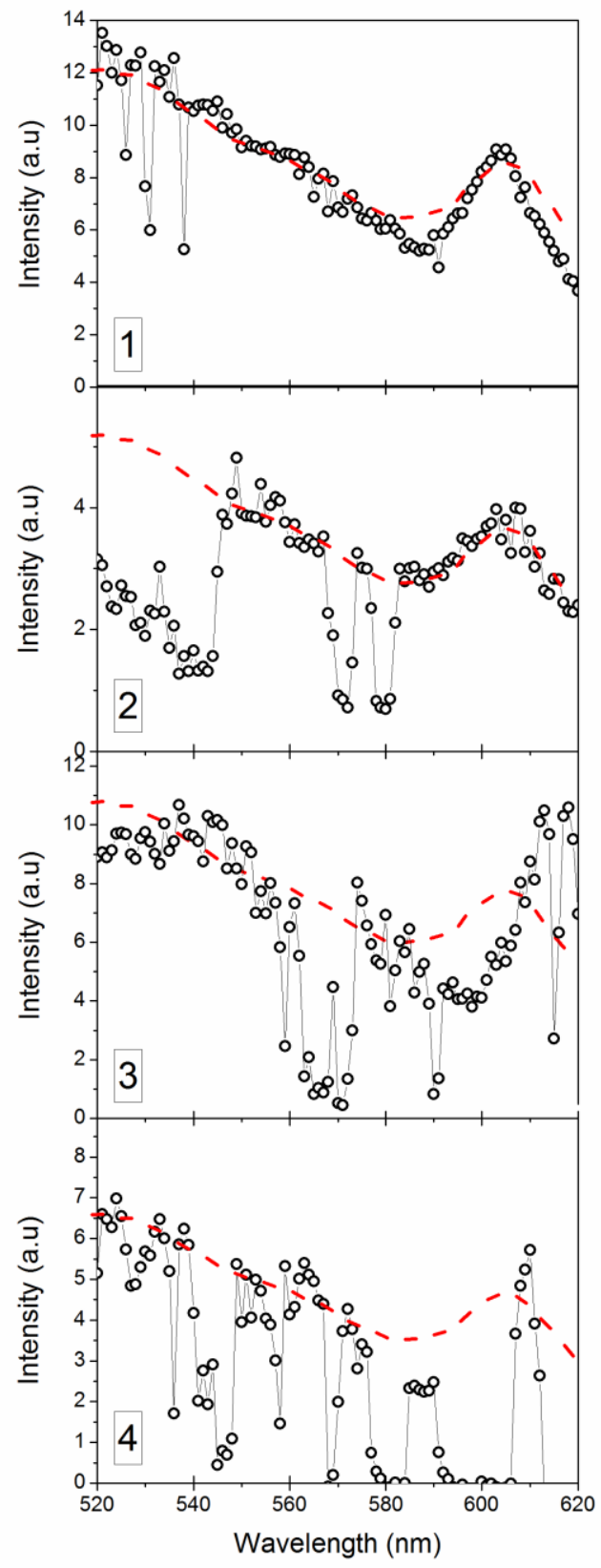

Figure 3.5 Single QD photoluminescence excitation spectra. For comparison, the ensemble excitation spectrum is shown as the red dashed line. The spectra show varying degrees of emission intermittencies visible as drops and gaps in the spectra. Especially in the wavelength region of the pronounced $1 \mathrm{~S}(\mathrm{e})$ $2 \mathrm{~S} 3 / 2(\mathrm{~h})$ transition the spectra show distinct differences between different quantum dots, reflecting the individual nature of each quantum dot. 
Comparing the excitation spectra of different quantum dots we find both striking similarities and some clear differences between the spectra. The single quantum dot spectra are always enveloped by the ensemble spectrum below $\sim 580$ nm (see all panels Figure 3.5, also Figure 3.4a). Besides dips and gaps due to blinking behavior on different timescales we see no significant differences between the single quantum dots or distinct individual features in this 'blue' region of the spectrum. This spectral region below $580 \mathrm{~nm}$ is exactly that region where a quasicontinuum of optical transitions was observed at cryogenic temperatures, corresponding to low lying energy barriers between distinct states predicted by the theory. It is therefore not surprising that, except for blinking events, no individual characteristics of the observed quantum dots can be identified at room temperature in this wavelength region.

The picture changes significantly when looking at the pronounced transition at $\sim 605 \mathrm{~nm}$, closer to the band edge, where we find clear differences in the shape, height and spectral position of this peak in the individual excitation spectra. Variations in the spectral position of this peak from individual quantum dots are attributed to differences in the size of the individual quantum dots. As expected for a single emitter study, we also find that the width of the transition is generally smaller than the width of the ensemble transition. This broadening in the ensemble results from the superposition of a large number of excitation spectra of single quantum dots of varying peak wavelength, creating an averaged ensemble spectrum that is spectrally broadened. Interestingly, we find some excitation spectra that do not show narrowed spectral features, e.g. Figure 3.5, panel 2, pointing towards the existence of an equivalent of spectral emission diffusion $[14,15]$ in the excitation spectrum and by inference, in the absorbance. Especially since we do not observe individual fingerprints in the quasicontinuum range, the observed differences in both the spectral position, and the relative height and shape differences for the $1 \mathrm{~S}(\mathrm{e})-2 \mathrm{~S} 3 / 2(\mathrm{~h})$ transition, reflects the individual characteristics of each single quantum dot. The shape and height of the peak from the $1 \mathrm{~S}(\mathrm{e})-2 \mathrm{~S} 3 / 2(\mathrm{~h})$ transition is determined by the coupling between these states. It has been shown that transitions close to the band edge show a fine structure splitting into sublevels due to the quantum dot crystal field, shape anisotropy, and confinement enhanced electron-hole exchange interactions [16]. Although considerably broadened at room temperature and hence invisible in the ensemble, the variation of the height and shape of the peak around $605 \mathrm{~nm}$ we have observed for individual quantum dots reflects details of these transitions. Since the differences in relative height of spectral features in the photoluminescence excitation spectrum represent the strength of the coupling between the 
ground and excited state of the individual quantum dots, it is likely that the observed differences in the individual excitation spectra reflect the differences in the photoluminescence quantum efficiency of individual quantum dots as seen before $[17,18]$.

In general, observed wavelength dependent changes in photo-luminescence can result from changes in either the absorbance or the photoluminescence quantum yield. Although excitation wavelength dependent changes in photoluminescence quantum efficiency have been discussed [19, 20], Tonti et al. were able to show that there is no intrinsic deviation between the excitation and absorbance spectra of quantum dots once all corrections and sample handling are properly performed [21]. This result implies that the photoluminescence quantum yield in CdSe quantum dot ensembles is independent of the excitation wavelength, and that analyzing the excitation spectra also allows one to draw conclusions about the absorbance spectra of single quantum dots. Following from this, the observed behavior directly results from the differences in the absorbance at different wavelengths of the quantum dots.

\subsubsection{Dark state dependence on excitation wavelength?}

The recorded data further enables the analysis on the influence of the excitation wavelength on the blinking of single quantum dots. Numerous studies on single quantum dots have shown complicated luminescence intermittency or blinking with power law statistics over many decades in time. In most of these studies a single excitation wavelength was used, and only recently has the first in-depth investigation of quantum dot blinking comparing a small number of different excitation wavelengths been published [22], where quantum dots showed a larger probability to have long lasting emissive states around the band-edge excitation range. These studies were based on the statistical analysis of different quantum dots, but a systematic analysis of the emission of individual emitters using different excitation wavelengths has not been reported yet.

The approach presented here makes it possible to study the response of individual emitters to changes in excitation wavelength over a broad range. Hence, increased photoluminescence intermittency for certain excitation wavelengths will result in systematically reduced emission intensity for this wavelength in our study. To start, we try to visualize the potential wavelength dependence by calculating the sum of the photoluminescence excitation spectra from all single quantum dots analyzed in our study and compared it to the ensemble spectrum recorded with a calibrated ensemble spectrometer (Figure 3.6). The summed photoluminescence 
excitation spectrum shows a number of interesting characteristics. Globally, blinking that was visible in the individual excitation spectra averages out, and the sum spectrum shows no explicit gaps where the intensity suddenly drops and then recurs. However there are clear differences between the ensemble photo-luminescence excitation spectrum and the summed excitation spectra from single quantum dots. On the short wavelength side, the sum spectrum increases much faster than the ensemble spectrum, while on the long wavelength side there is good agreement between the positions of the pronounced $1 \mathrm{~S}(\mathrm{e})-2 \mathrm{~S} 3 / 2(\mathrm{~h})$ transition. The difference on the short wavelength side of the sum spectrum is not apparent in the individual spectra and can be understood from the details of how the single emitter excitation spectrum was recorded, that is, by considering that the excitation wavelength was always scanned from short to long wavelengths. As the transition to dark states is excitation-light driven, the probability to find a single quantum dot in a non-emitting state is minimal at the start of the experiment. Over time - which translates to longer excitation wavelengths in our experiment - the probability to find a quantum dot in a long-lived dark state increases. In the extreme case, a transition to a dark state occurs

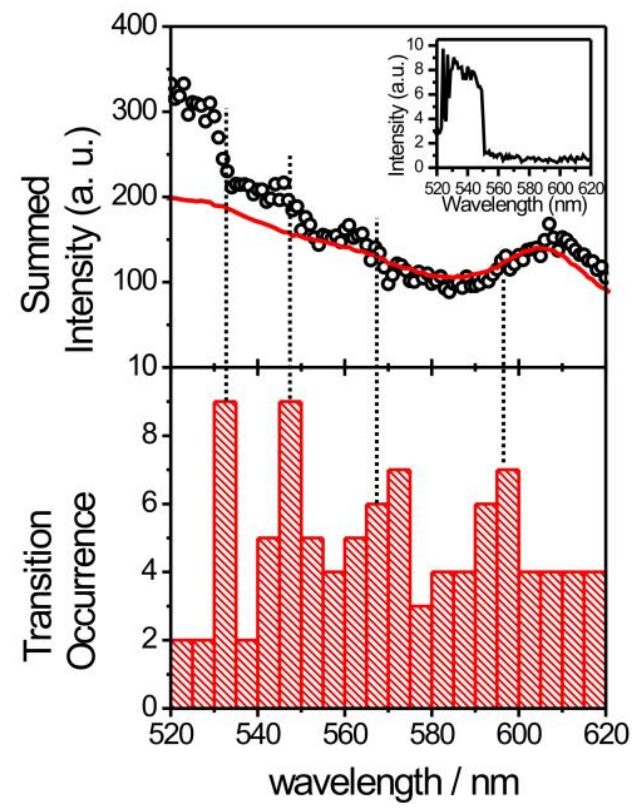

Figure 3.6 Single quantum dot excitation spectra reveal distinct excitation wavelengths with increased probability for a dark-state transition. Upper panel: sum spectrum of 48 excitation spectra of individual quantum dots (circles). Red: ensemble excitation spectrum. Inset: Single quantum dot excitation spectra undergoing a transition to a dark state. Lower panel: Histogram of transitions to a long lived dark-state. 
and photoluminescence is not regained before the end of the experiment (Figure 3.6, inset). Thus, shorter excitation wavelengths are overweighted in the sum spectrum in the excitation scheme used, which was dictated by the monochromator used for these experiments that allows only for scanning the excitation from short to long wavelengths. One promising way to overcome this limitation in future experiments is to use an acousto-optical tunable filter (AOTF) for fast wavelength selection and arbitrary wavelength scanning, averaging out the dips and gaps in the spectra.

However, not all differences between the sum spectrum and the ensemble spectrum can be explained by the details of the excitation scheme used. If the probability for a transition to a long-lived dark state is independent of the excitation wavelength, a steady decline of the summed single quantum dot excitation spectrum approaching the spectral shape of the ensemble spectrum is expected when both spectra are normalized to the long wavelength edges of the spectra. Indeed, we find good agreement between the sum and ensemble spectra for excitation wavelengths beyond $\sim 580 \mathrm{~nm}$, which suggests only a minor influence from transitions to dark states which do not recover during the entire data acquisition time in this wavelength range. On the other hand we find large deviations between the sum and ensemble spectra for excitation wavelengths below $\sim 580 \mathrm{~nm}$. This observation is consistent with reports that excitation in the band gap area results in little blinking compared to excitation above the band gap [22]. However, we do not see a smooth decline of the excitation sum spectrum, but observe what appear to be a number of steps ( $\sim 530$ $\mathrm{nm}, \sim 550 \mathrm{~nm}, 565 \mathrm{~nm}$, and $595 \mathrm{~nm}$ (less prominent); see Figure 3.6). These drops in the sum spectrum indicate that at the associated excitation wavelengths either very bright emitters switched off, or that statistically an increased probability of a transition to a dark state exists. To verify if the observed steps in the sum spectrum point towards a molecular mechanism underlying the increased probability for a transition to a dark state, we created a histogram of the wavelengths for which a transition to a dark state could be observed at the single emitter excitation spectra (Figure 3.6b). The histogram shows increases in the occurrence of a dark state transition for the excitation wavelengths $\sim 532 \mathrm{~nm}, \sim 548 \mathrm{~nm}, \sim 570 \mathrm{~nm}$ and $\sim 595 \mathrm{~nm}$. All four peaks are correlated to a signature in the sum spectrum. To estimate the statistical significance of the obtained probability distribution, we determined the p-value assuming equal probability of a dark state transition for all power normalized excitation wavelengths. We obtain a p-value of 0.06 indicating a low probability of $\sim 6 \%$ that the observed distribution originates from a random distribution of dark state transitions. However, the sample number is too low $(\mathrm{N}=96)$ to conclude that 
the observed steps in the sum spectrum are caused by increased probability of a transition to a dark state at the associated excitation wavelength. A more systematic measurement is required to test the relation between excitation wavelength and blinking behavior, which is the scope of Chapter 5, where blinking statistics are measured using a larger number of quantum dots, and at 14 different excitation wavelengths that are broadly distributed across the spectral excitation range.

\subsection{Conclusion}

We have recorded for the first time single emitter excitation spectra at room temperature. The required spectrally narrow excitation over a wide spectral range of $100 \mathrm{~nm}$ was realized by using a monochromator to select the excitation wavelength from a supercontinuum white light source. The suitability of our approach and its potential were demonstrated by studying single quantum dots. The single emitter nature of our quantum dot samples was confirmed by photon antibunching experiments. Analysis of the single quantum dot excitation spectra gave access to unexplored details of this class of emitters. The excitation spectra showed no clear individual features for excitation wavelengths well above the band gap, but exhibited large differences for transitions close to the excitonic peak. We found variations in the width and the spectral position of transitions for individual quantum dots, representing the individual differences in coupling strength for the band-edge transition and reflecting the fine structure splitting into energetic sublevels of the individual quantum dots. For the CdSe nanocrystals investigated, we found indications for an increased probability for a transition to long-lived dark states at specific excitation wavelengths, although a more systematic measurement is required to study the exact relation between excitation wavelength and quantum dot blinking. The excitation spectrum shown in the top panel of Figure 3.5 is very intense and shows only minor signs of blinking, and is based on the detection of $\sim 2 \cdot 10^{5}$ photons emitted from the sampled quantum dot. This number of detected photons is well comparable with the average number of photons that can be detected from organic fluorophores [23, 24] and suggests that single emitter excitation spectroscopy can also be used for classes of emitters other than the very photostable quantum dots analyzed in this chapter.

In the next chapter, the realization of both excitation and emission spectroscopy on single organic dyes will be discussed. In addition to the work described in the current chapter, we use the band maxima of the absorption and the lower-energy (red-shifted) emission to systematically record the Stokes shift of single emitters. 


\section{REFERENCES}

1. Chong, S., W. Min, and X.S. Xie, Ground-state depletion microscopy: Detection sensitivity of single-molecule optical absorption at room temperature. The Journal of Physical Chemistry Letters, 2010. 1(23): p. 3316-3322.

2. Gaiduk, A., et al., Room-temperature detection of a single molecule's absorption by photothermal contrast. Science, 2010. 330(6002): p. 353-356.

3. Kukura, P., et al., Single-molecule sensitivity in optical absorption at room temperature. The Journal of Physical Chemistry Letters, 2010. 1(23): p. 3323-3327.

4. Vermeulen, P., L. Cognet, and B. Lounis, Photothermal microscopy: Optical detection of small absorbers in scattering environments. Journal of Microscopy, 2014. 254(3): p. 115-121.

5. Ambrose, W.P. and W.E. Moerner, Fluorescence spectroscopy and spectral diffusion of single impurity molecules in a crystal. Nature, 1991. 349(6306): p. 225227.

6. Orrit, M. and J. Bernard, Single pentacene molecules detected by fluorescence excitation in a p-terphenyl crystal. Physical Review Letters, 1990. 65(21): p. 27162719.

7. Klimov, V.I., Spectral and dynamical properties of multilexcitons in semiconductor nanocrystals, in Annual review of physical chemistry. 2007, Annual Reviews: Palo Alto. p. 635-673.

8. Empedocles, S.A., D.J. Norris, and M.G. Bawendi, Photoluminescence spectroscopy of single cdse nanocrystallite quantum dots. Physical Review Letters, 1996. 77(18): p. 3873-3876.

9. Htoon, H., P.J. Cox, and V.I. Klimov, Structure of excited-state transitions of $i$ ndividual semiconductor nanocrystals probed by photoluminescence excitation spectroscopy. Physical Review Letters, 2004. 93(18): p. 187402.

10. Basché, T., et al., Photon antibunching in the fluorescence of a single dye molecule trapped in a solid. Physical Review Letters, 1992. 69(10): p. 1516-1519.

11. Lounis, B., et al., Photon antibunching in single cdse/zns quantum dot fluorescence. Chemical Physics Letters, 2000. 329(5-6): p. 399-404.

12. Zwiller, V., et al., Single quantum dots emit single photons at a time: Antibunching experiments. Applied Physics Letters, 2001. 78(17): p. 2476-2478.

13. Fisher, B.R., et al., Emission intensity dependence and single-exponential behavior in single colloidal quantum dot fluorescence lifetimes. Journal of Physical Chemistry B, 2004. 108(1): p. 143-148.

14. Stracke, F., et al., Correlation of emission intensity and spectral diffusion in room temperature single-molecule spectroscopy. Chemphyschem, 2005. 6(7): p. 12421246.

15. Stracke, F., et al., Intrinsic conformer jumps observed by single molecule spectroscopy in real time. Chemical Physics Letters, 2000. 325(1-3): p. 196-202.

16. Htoon, H., et al. Interplay of rabi oscillation and quantum interference in semiconductor quantum dots. in Quantum Electronics and Laser Science Conference, 2002. QELS '02. Technical Digest. Summaries of Papers Presented at the. 2002.

17. Biebricher, A., M. Sauer, and P. Tinnefeld, Radiative and nonradiative rate fluctuations of single colloidal semiconductor nanocrystals. The Journal of Physical Chemistry B, 2006. 110(11): p. 5174-5178. 
18. Brokmann, X., et al., Measurement of the radiative and nonradiative decay rates of single cdse nanocrystals through a controlled modification of their spontaneous emission. Physical Review Letters, 2004. 93(10): p. 107403.

19. Hoheisel, W., et al., Threshold for quasicontinuum absorption and reduced luminescence efficiency in cdse nanocrystals. The Journal of Chemical Physics, 1994. 101(10): p. 8455-8460.

20. Rumbles, G., et al., Anomalies in the linear absorption, transient absorption, photoluminescence and photoluminescence excitation spectroscopies of colloidal inp quantum dots. Journal of Photochemistry and Photobiology A: Chemistry, 2001. 142(2-3): p. 187-195.

21. Tonti, D., F. van Mourik, and M. Chergui, On the excitation wavelength dependence of the luminescence yield of colloidal cdse quantum dots. Nano Letters, 2004. 4(12): p. 2483-2487.

22. Knappenberger, K.L., et al., Excitation wavelength dependence of fluorescence intermittency in cdse/zns core/shell quantum' dots. Nano Letters, 2007. 7(12): p. 3869-3874.

23. Deschenes, L.A. and D.A. Vanden Bout, Single molecule photobleaching: Increasing photon yield and survival time through suppression of two-step photolysis. Chemical Physics Letters, 2002. 365(5-6): p. 387-395.

24. Lord, S.J., et al., Azido push-pull fluorogens photoactivate to produce bright fluorescent labelst. The Journal of Physical Chemistry B, 2009. 114(45): p. 1415714167. 


Chapter 4

\section{Excitation spectra and Stokes shift measurements of single organic dyes at room-temperature}

In the previous chapter we demonstrated instrumentation capable of recording excitation spectra of single emitters at room temperature. In this chapter, we go one step further by recording excitation spectra of single, polymer embedded, perylene dye molecules, an organic fluorophore of a class of fluorophores similar to those being used in modern fluorescence microscopy studies. In addition, we recorded emission spectra of the same single emitters to acquire a full spectral dataset of a single emitter. Having both the excitation and emission spectra allows one to study in full spectroscopic detail at the single molecule level phenomena such as spectral diffusion, which so far have only been studied using emission spectra. Moreover, the energy difference of the band maxima can be used to systematically study the Stokes shift, a parameter that has not yet been recorded for single emitters. Due to the inability to determine the Stokes shift on the single molecule level, the Stokes shift has often been assumed to be constant in single molecule studies. Our results show that the Stokes shift varies from molecule to molecule, and causes a larger spectral heterogeneity of the emission spectra compared to the excitation spectra.

This chapter has been accepted for publication in The Journal of Physical Chemistry Letters. 


\subsection{Excitation spectra of single organic fluorophores}

In the previous chapter we described how we developed a method to record excitation spectra from a single emitter [1], and used quantum dots to demonstrate the accessibility to a broad range of excitation wavelengths. Quantum dots are very bright and photostable emitters, which was useful for the qualification of single emitter excitation spectroscopy in the previous chapter. In practice, the most commonly used class of fluorophores are organic fluorophores, being small molecules and bright at the same time, properties that are particularly useful in the life sciences to provide minimal steric hindrance for intracellular molecules and at the same time reveal great detail about processes inside the cells. A large set of organic probes exist that each have their own specialty or specific sensitivity to the local nanoenvironment. However, organic fluorophores are less bright than quantum dots and in general photobleach faster. Therefore, recording excitation spectra of organic fluorophores becomes practically more challenging, because it requires both fast and efficient data acquisition to be able to acquire the excitation spectrum before the fluorophore photobleaches; this effectively requires faster wavelength scanning and optimal detection sensitivity.

In this chapter, we show how we recorded for the first time excitation spectra of single organic emitters at room temperature. The organic fluorophores we used are single perylene dyes, which interestingly are known to show a distribution of spectral shifts in the emission spectra, which varies from emitter to emitter and fluctuates over time [2-4], a phenomenon often called spectral diffusion [5, 6]. To date, spectral variations and spectral diffusion have been reported for various classes of embedded fluorophores, including organic dyes in polymer matrices [7-10] and fluorescent proteins [3, 11]. In fluorescent proteins the chromophore is embedded within the protein and it has been shown that the flexibility of the chromophore surrounding determines the widths of the spectral spread [12].

Since excitation and emission transitions are connected to the same ground and excited state, spectral diffusion is expected to be visible in the excitation spectra as well. The same spectral heterogeneity is expected for the excitation spectra when assuming that spectral shifts solely arise from differences in the energy gap between the ground state and the excited state. In the absence of detailed insights, a number of studies used this "constant Stokes shift assumption" [5, 9, 13], assuming that the Stokes shift is not affected by the differences in the nanoenvironment.

To compare the spectral heterogeneity of the excitation and emission spectra, we additionally recorded emission spectra of the same single emitters, having a full spectral dataset of a single emitter. We determine the energies of the band maxima 
of the excitation and emission spectra to determine the distributions of energy shifts, which reflect the spectral heterogeneity of the excitation and emission spectra, to see if the constant Stokes shift assumption still holds. If the widths of the two distributions match, the Stokes shift remains constant, but a varying Stokes shift would give a seemingly wider distribution for the emission spectra compared to the excitation spectra, because in that case the variation in Stokes shift additionally contributes to the observed heterogeneity of the emission spectra.

\subsection{Stokes shift}

The Stokes shift is also one of the key parameters of fluorescence that has not yet been accessed on the single molecule level. The Stokes shift is the difference in energy between the band maxima of the absorption and the lower energy (red shifted) emission [14]. From a practical perspective, the Stokes shift is key to any use of fluorescence-based methods, since it allows the easy separation of emitted fluorescence from excitation light by using appropriate optical filters. The loss in energy between excitation and emission is caused by non-radiative dissipation processes that are on the one hand characteristic for the fluorophore, but also give information on the excited state of the fluorophore. The Stokes shift is also affected by excited state processes. For example, if the dipole moments of the excited state and ground state differ, the Stokes shift will increase with solvent polarity (MatagaLippert theory) [14-16], due to solvent relaxation processes after excitation. On the ensemble level, the Stokes shift has proven to be a sensitive parameter reporting on the polarity, mobility and flexibility of the nanoenvironment of the fluorophores [17].

The Stokes shift also plays an important role in the phenomenon of spectral heterogeneity observed in room temperature single molecule spectroscopy. In essence, the spectral position of the emission of single fluorophores that interact with their environment varies from emitter to emitter and fluctuates over time [2, 4-7, 9, 12, 18-21]. From ensemble studies it is well known that the spectral properties of a fluorophore, that is the absorbance as well as the emission, strongly depend on external factors like solvent polarity, viscosity, the presence of certain ions, etc. [22]. The observed single molecule spectral heterogeneity has been attributed to heterogeneity in the conformation of macromolecules, and to variations in the guesthost coupling between the fluorophore and its nano-environment that determine the exact photophysical parameters of the fluorophore. However, it remains an open question whether the observed variations in single molecule spectral emission positions arise from environment-induced synchronous shifts of the absorbance and 

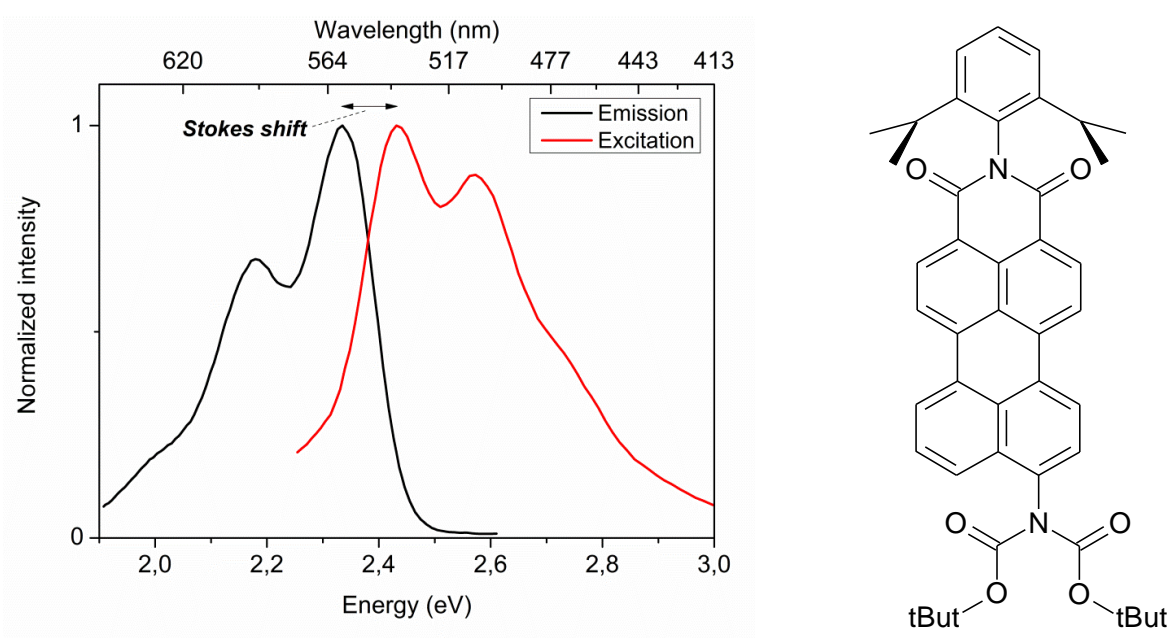

Figure 4.1 (left) Ensemble excitation and emission spectra of perylene dissolved in toluene. The excitation and emission spectra show characteristic peaks. The Stokes shift is by definition the energy difference between the excitation peak with the lowest energy and the emission peak with the highest energy. The Stokes shift of the studied perylene derivative in toluene is determined to be $96 \mathrm{meV}\left(\sim 800 \mathrm{~cm}^{-1}\right)$. (right) Chemical structure of perylene

emission spectra, or from differences in the Stokes shift between emitters, or from combinations of both. In the absence of detailed insights, a number of studies have used the "constant Stokes shift assumption" [5, 9, 13], that is, that the Stokes shift is not affected by the differences in the nano-environment.

Since we determine the peak energies of both the excitation and emission band maxima of the single perylene dyes, we are able to measure the Stokes shift of single organic fluorophores, and study the distribution of the Stokes shifts to explicate the "constant Stokes shift assumption".

\subsection{Experiment}

We recorded single molecule emission and excitation spectra of the perylene derivative N,N-di-(tert-butoxycarbonyl)-9-amino-N-(2,6-diisopropylphenyl)perylen3,4-dicarboximide in a polystyrene (PS) polymer matrix. ${ }^{3}$ The emission spectra of polymer-embedded perylene molecules are well-studied and have been found to show molecular heterogeneity and spectral diffusion [6, 9, 19]. In addition, perylenes are known to be exceptionally photostable, allowing us to acquire both an excitation

\footnotetext{
${ }^{3}$ The sample was prepared using a similar procedure as described in chapter 2 , except that the spincoated solution contained $0.8 \mathrm{~g}$ PS in $100 \mathrm{ml}$ of toluene.
} 
and an emission spectrum from the same molecule to determine the Stokes shift. The ensemble spectra show distinct peaks (see Figure 4.1) that are characteristic for perylenes. In this chapter, we use energy scales rather than wavelength scales to present the data, to simplify the anticipated calculation of the Stokes shift from the band maxima later on. For reference, the wavelength scale (inverse of energy) is included on the top axis. The Stokes shift, by definition the energy difference between the excitation peak with lowest energy and the emission peak with the highest energy, is $96 \mathrm{meV}\left(\sim 800 \mathrm{~cm}^{-1}\right)$ for the ensemble toluene solution. We raster scan this sample to localize the single isolated emitters. The perylene dyes typically show a stable emission pattern with a sparse amount of blinking (see Figure 4.2). After locating the single emitters, we select the ones that are fully spatially isolated and record both the excitation and emission spectra at the same location. To do so, we use two filter sets that can be interchanged in-between the spectral recordings. To measure excitation spectra we use an SP01-561RU filter in combination with LP02561RU and FF01-582/15 filters (all filters from Semrock Inc., NY, USA) and excite the emitters using a wavelength scanning range from $461 \mathrm{~nm}$ to $561 \mathrm{~nm}(2.2 \mathrm{eV}$ to $2.75 \mathrm{eV}$ ) with an integration time of $200 \mathrm{~ms}$ per excitation wavelength. Instead of using a scanning grating monochromator, we tuned the excitation wavelengths using an acousto-optical tunable filter (AOTF, Crystal Technologies, West Chester PA, USA)
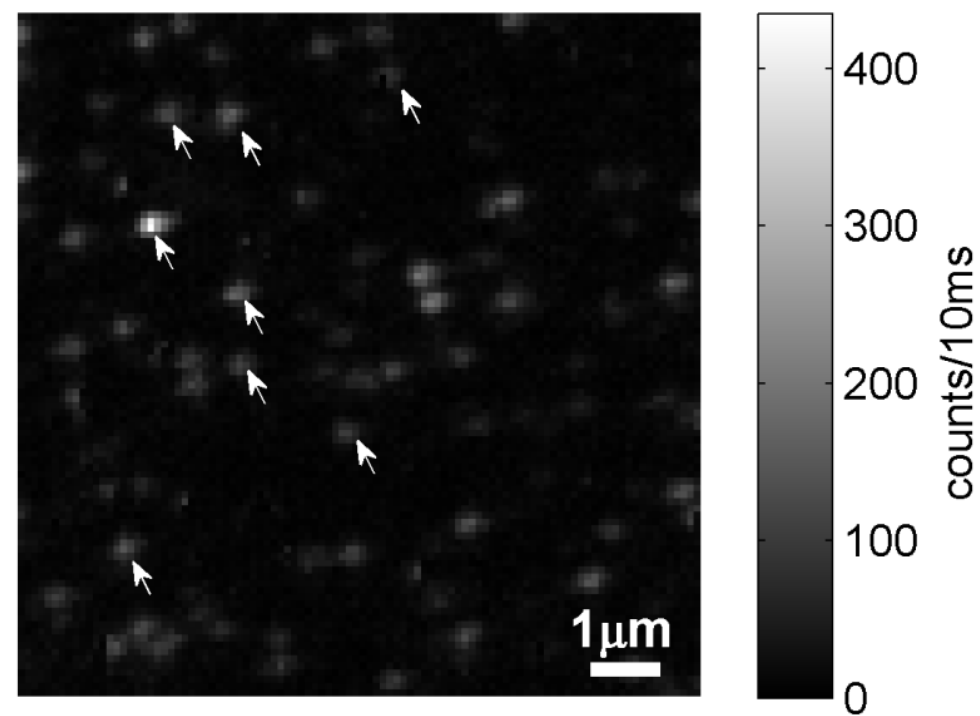

Figure $4.210 \mathrm{x} 10 \mathrm{\mu m}^{2}$ area-scan of the perylene sample. The arrows indicate the isolated emitters that are typically selected to record excitation and emission spectra. 
to select any desirable wavelength in the visible spectral band from a Fianium supercontinuum laser source (SC400-PP). The AOTF allows for faster wavelength switching compared to the monochromator described in the previous chapter, which is suitable for recording excitation spectra of fluorophore types that are prone to photobleaching fast, like organic fluorophores. To measure emission spectra we use an FF02-447/60 filter in combination with a BLP01-488R long-pass filter and excite the emitters at a wavelength of $470 \mathrm{~nm}$, and record the emission from $\sim 500 \mathrm{~nm}$ and longer wavelengths $(<2.5 \mathrm{eV})$, using a custom-built prism spectrograph (see chapter 2). The filterset data are shown in Figure 4.3, and ensure that excitation and emission light are fully isolated from each other and do not hinder the detection of the emission light coming from the single emitters.

After recording the single emitter spectra, we apply a background correction to the excitation and the emission spectra by subtracting spectra that are recorded at areas between the single emitters. We did not correct for wavelength dependent differences in the sensitivity of the setup, which explain some of the differences in the spectral shape of the bulk emission spectra and the single molecule spectra. Furthermore, we apply a photon flux density correction for the excitation spectra,

$$
\Phi_{d}=\frac{P}{E_{p h} \cdot A} \sim \frac{P(\lambda)}{\lambda}
$$

where $\Phi_{d}$ is the photon flux density, $P$ the power at wavelength $\lambda, E_{p h}$ the energy of a photon and $A$ the area of the diffraction-limited focus spot. The photon flux density correction is different from the correction used for the quantum dot excitation spectra in the previous chapter, where a reference ensemble spectrum was used to
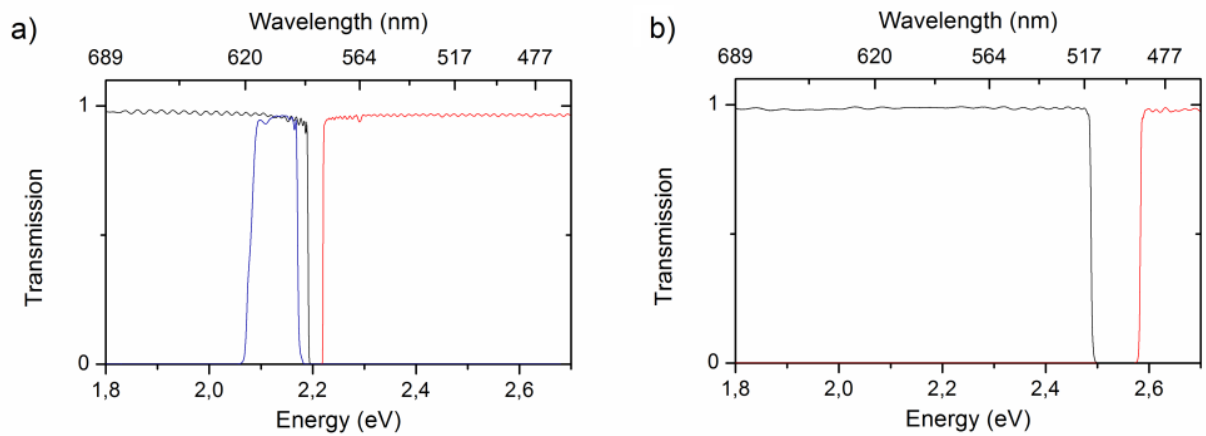

Figure 4.3 (a) Transmission curves of the filter set used for recording excitation spectra, and likewise (b) for recording emission spectra, obtained from the manufacturer specifications. All filters show a smooth transmission curve. 
calibrate the setup. The photon flux density correction does not require a reference spectrum and considers wavelength scaling of the excitation illumination spot at the sample, which is predictable using Abbe's diffraction limit for diffraction limited spots. Prior to the experiment, the excitation power $P$ is measured at the sample plane using a calibrated power-meter. The photon flux density correction is robust because it does not depend on the quality (shape and wavelength calibration) of a reference spectrum, and is therefore a highly recommended procedure for future experiments.

\subsection{Excitation and emission spectra of single organic emitters}

From the total dataset of 435 molecules, we successfully recorded 100 excitation and 80 emission spectra. Due to blinking and bleaching of emitters, not all excitation and emission spectra were fully acquired. In particular, the excitation spectra are vulnerable to blinking, because these spectra are essentially time-series of the emission intensity measurements. Furthermore, the spectra could vary over time, which is expected to smear and broaden the shape of the spectra. For our analyses, we only consider successfully recorded spectra that show the distinct peaks that are characteristic for perylene dyes, and thus are not expected to have displayed significant intrinsic heterogeneity. Representative examples of single molecule excitation and emission spectra are shown in Figure 4.4.

For all recorded single emitter excitation and emission spectra we observe the characteristic shapes as expected from the ensemble spectra, but show moleculeto-molecule variations in spectral positions and shapes. The variations in the spectral shape reflect molecule-to-molecule differences in the Franck-Condon factors for excitation transitions, representing the excitation analogue to the previously observed spectral variations in the emission spectra [6]. The variations in the excitation spectral position relate to differences in the energy gap between ground state and excited state. Note that alterations in the Stokes shift, related to relaxation and reorientation of the nanoenvironment, after the excitation event, do not contribute to the distribution of excitation spectral positions.

For the emission spectra (Figure 4.4b), we observe heterogeneity in spectral positions as well as in the shape of the spectra in agreement with previous reports [6, 9, 19, 23]. Analogous to the differences in the excitation spectral shapes, the differences in spectral shape of the emission spectra reflect differences in the FranckCondon factors for the emission transitions. The emission spectral position however depends on the energy gap between ground state and excited state upon excitation, 
as well as on the radiationless dissipation and loss of energy due to possible reorganization of the fluorophore nanoenvironment when in the excited state.

To quantify the spectral variations, we constructed a histogram of the energies of the lowest energy excitation peaks of all 100 successfully recorded excitation spectra (Figure 4.4c), and likewise, a histogram of the highest energy emission peak energies of all 80 successfully recorded emission spectra (Figure $4.4 \mathrm{~d})$. The mean peak energies of the excitation and emission distributions are $2.38 \mathrm{eV}$ and $2.25 \mathrm{eV}$, respectively. These energies are slightly lower than the spectral

a)

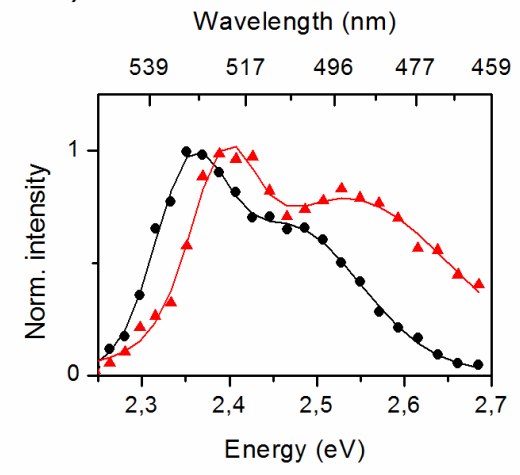

c)

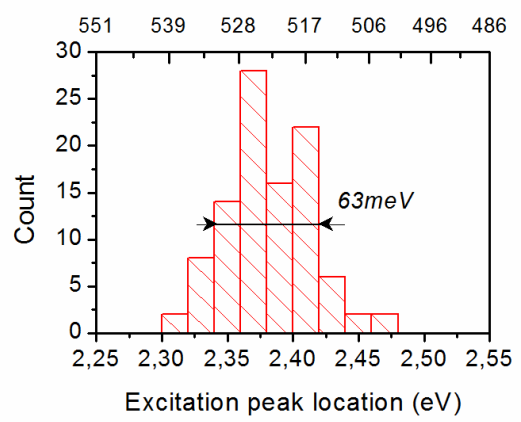

b)

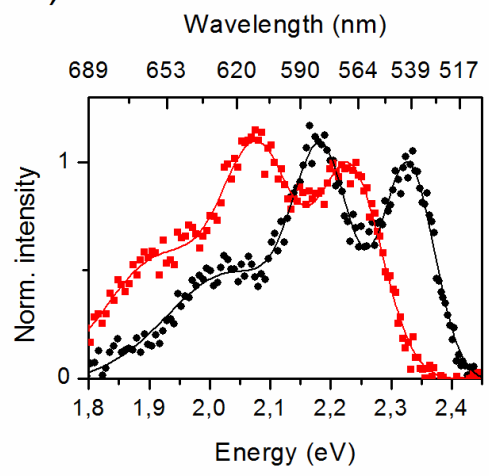

d)

Wavelength $(\mathrm{nm})$

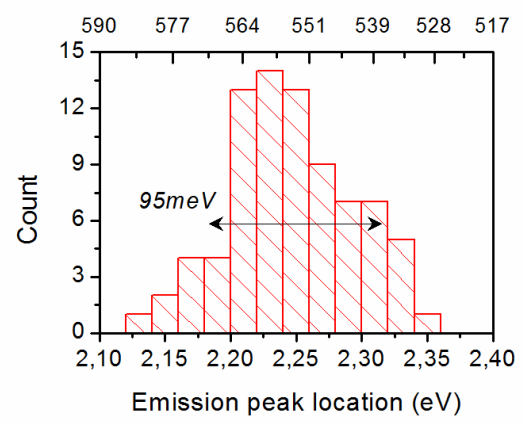

Figure 4.4 Examples of recorded excitation (a) and emission spectra (b) from different emitters Solid lines are a guide to the eye, based on double and triple Gaussian fits to the data. For both the excitation and the emission spectra we observe molecule to molecule differences in the shape and the spectral position of the recorded spectra. We construct histograms of the excitation and emission peak energies of the band maxima, for all successfully recorded excitation and emission spectra. (c) The center of the excitation peak energy distribution is found to be at $2.38 \mathrm{eV}$ and the width of the distribution is $63 \mathrm{meV}$. (d) The emission peak energy distribution is centered around $2.25 \mathrm{eV}$, and the width of the distribution is $95 \mathrm{meV}$. 
peak energies of the ensemble spectra, which are at $2.43 \mathrm{eV}$ and $2.34 \mathrm{eV}$. These differences with the bulk data highlight the important role of the exact nature of the nanoenvironment of the emitters. We suspect that the spectral differences we observe between solution and single molecule experiments arise from the thin film embedding.

As expected, both peak energy distributions appear normally distributed, since random fluctuations of molecular properties cause spectral variations that are typically characterized by a normal distribution of the observed quantity [6]. As an additional test, we apply a Shapiro-Wilk (S-W) normality test to both distributions. The S-W test is a powerful test that works specifically well with small sample sizes ( $n \leq 50)$, but can also be applied to larger sample sizes up to $n=2000$ [24]. Generally, a p-value $<0.05$ for the tested distribution is considered as a proof that the distribution is significantly different from a normal distribution while a p-value of 1 indicates a perfect match with a normal distribution [25]. For the distribution of excitation peak energies we find a p-value of 0.35 and for the distribution of the emission peak energies a p-value of 0.63 , meaning that the $\mathrm{S}$-W test does not consider both distributions to significantly differ from a normal distribution.

The widths of the distributions can give initial insights into whether the Stokes shift between the molecules is constant or if the Stokes shift varies. One would expect the widths of the energy distributions of the excitation maximum and the emission maximum to be identical in the case that the Stokes shift is constant for all molecules. Variable Stokes shifts, e.g. due to additional relaxation processes characteristic for the individual molecule that change the energy of the excited or ground state while the molecule is in the excited state, will add to the distribution sampled in excitation, such that the emission distribution will be wider than the excitation distribution.

We find the characteristic signature of molecule-to-molecule variations of the Stokes shift in our data. The width $(2 \sigma)$ of the excitation distribution is $63 \mathrm{meV}$ and hence significantly smaller than the width of the emission distribution, which is $95 \mathrm{meV}$. The distribution widths of the excitation and emission peak energy show the exact energy gaps between the ground and excited state for the excitation and emission process. The larger distribution width of the emission peak energy histogram compared to the distribution width for the excitation spectra, however, shows that there is an additional contribution from processes related to the excited state that contribute to the spectral variations of the emission spectra. This difference in the distribution widths strongly indicates that the Stokes shift of the single emitters shows variations from molecule to molecule. 


\subsection{Stokes shift of single emitters}

Using our data we can go one step further and directly study correlations of excitation and emission spectral position and determine the Stokes shift for single emitters. For 59 molecules we obtained complete excitation and emission spectral datasets of the same molecule, from which the Stokes shift of the single molecule could be determined. Typical spectral datasets are shown in Figure 4.5a.

The spectral heterogeneity is clearly visible in the excitation and emission spectra as the peak energies vary from emitter to emitter. Interestingly, the excitation and corresponding emission spectra in figure 4.5a (top three panels) appear to be shifting synchronously in the same direction of energy, while the Stokes shift does not show noticeable differences. However, for some molecules we find stronger deviations in the Stokes shift (see lowest panel). To examine the correlation between excitation and emission spectral shifts we plot the excitation peak energy versus the emission peak energy for all successfully recorded full spectral datasets (figure $4.5 \mathrm{~b}$ ). We observe a clear trend that higher excitation peak energies are commensurate with higher emission peak energies. The data points globally follow a line with a slope of 1 (dashed line as guide to the eye in figure 4.5b) in the scatterplot, showing that the excitation peak energy and emission peak energy are correlated, suggesting that the variations in the energy gap of the ground and excited state are a main contributor to the observed spectral variations of the emission spectra. However, the data points form a cloud rather than a line, hinting at a contribution from an additional factor, which is likely to be related to the distribution of the Stokes shift.

In a next step we determined the Stokes shift for each of the single molecules from the excitation and emission spectral peak energies, with an estimated uncertainty of $10 \mathrm{meV}$, and constructed a Stokes shift histogram (figure 4.5c). We find the Stokes shift being distributed around $128 \pm 39 \mathrm{meV}$ (standard deviation). This is in agreement with the Stokes shift that is found for the ensemble spectra $(96 \mathrm{meV})$. The Stokes shift histogram clearly shows the distribution of the Stokes shift, reflecting differences in the polarity and flexibility of the individual nanoenvironment that contributes to the Stokes shift. It is thus the combination of both variations in the energy gap between the ground state and the excited state and the variations in the Stokes shift that result in spectral variations observed in the emission spectra. 
a)

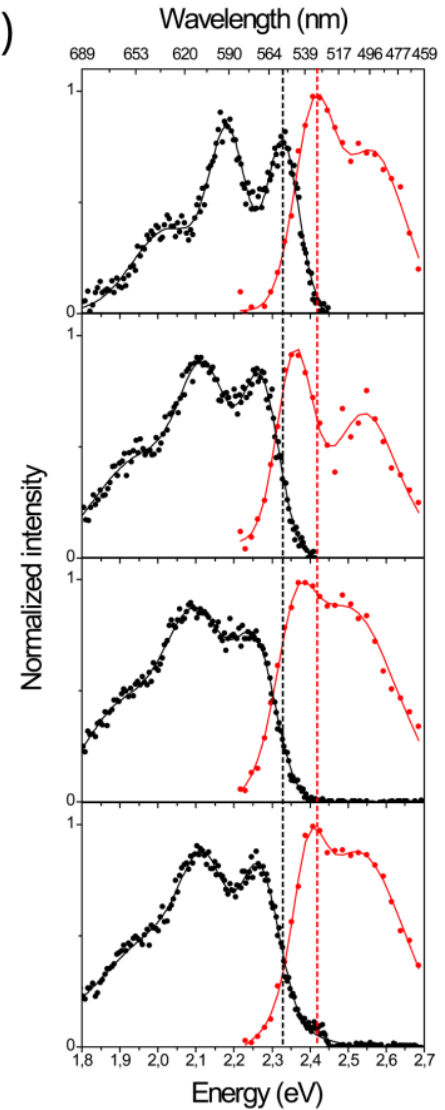

b)

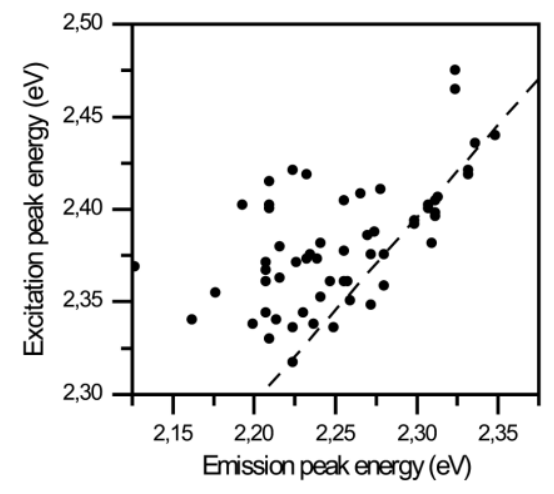

C)

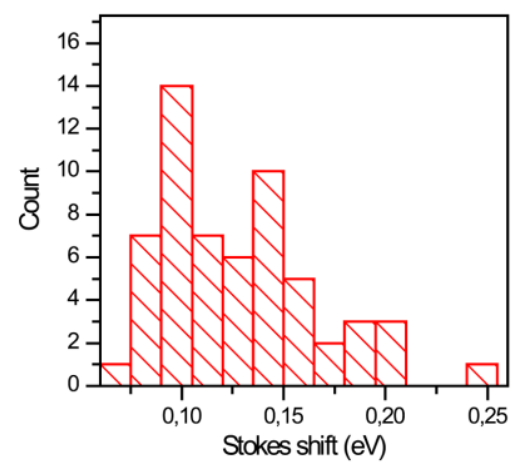

Figure 4.5 (a) Spectral datasets of four different single emitters. The intensities are normalized by maximum intensity and filtered using a Gaussian filter. The emission and excitation spectra are fitted with triple and double Gaussian functions, respectively, as guides to the eye (solid lines). The dashed lines indicate the emission (black) and excitation (red) peak energies of the upper spectral dataset, providing a guide to the eye to show the spectral shifts. (b) Excitation peak energy versus emission peak energy. The dashed line is a guide to the eye that indicates a correlated shift between excitation peak energy and emission peak energy, and has a slope of 1 . (c) Stokes shift distribution. We used the Stokes shift at the peak of the distribution (96meV) to produce the dashed line in (b) and indicate the predicted location in the scatterplot for datapoints that have a Stokes shift of $96 \mathrm{meV}$.

For the samples studied here, the Stokes shift appears not to follow a normal distribution as we would have expected. We perform a S-W normality test on the Stokes shift distribution, which gives a p-value of 0.002, indicating that the Stokes shift distribution is significantly different from a normal distribution. Interestingly, if we analyze the Stokes shifts using a k-means cluster analysis to find multiple groups, a good agreement is found with the Stokes shift histogram using three groups. The 
mean Stokes shifts of those three groups are $96 \pm 12 \mathrm{meV}, 142 \pm 12 \mathrm{meV}$ and $198 \pm 20 \mathrm{meV}(\sigma)$, which match the peak energies and peak widths at Figure $4.5 \mathrm{c}$. In addition, to support the observation of the three groups, we identify the data points in Figure 4.5b that correspond to these groups and determine the linear correlation coefficient of the data points within each group. We observe a strong increase in Pearson's linear correlation coefficient between the excitation and emission peak energies when comparing before $\left(\mathrm{r}_{\mathrm{all}}=0.59 \pm 0.08, \mathrm{~N}_{\mathrm{all}}=59\right)$ and after $\left(\mathrm{r}_{1}=0.95 \pm 0.02\right.$, $\left.\mathrm{N}_{1}=29 ; \mathrm{r}_{2}=0.93 \pm 0.05, \mathrm{~N}_{2}=21 ; \mathrm{r}_{3}=0.85 \pm 0.10, \mathrm{~N}_{2}=9\right)$ subdividing the points into groups, even though the statistical sample decreases. Moreover, the corresponding errors (obtained through bootstrapping [26]) of the first two groups also decrease after subdivision, supporting the increased linear correlation of the datapoints for the first two groups. We speculate that at least the first two of the three peaks in the Stokes shift histogram represent different, preferred, dissipation pathways that can be formed by the perylene embedded in polystyrene. However, the nature of the pathways is still unknown.

\subsection{Conclusion}

We have determined excitation spectra and Stokes shifts of single organic fluorophores for the first time to the best of our knowledge. Our data gives new insights into the phenomenon spectral heterogeneity of the single molecule fluorescence emission spectra. We find a distribution of the spectral positions of the excitation spectra, providing evidence of molecule-to-molecule differences in the energy gap between ground and excited states. We further find a distribution of the Stokes shifts on the single molecule level, related to differences in the excited state relaxation before emission, which we attribute to local variations in the polarity and flexibility of the emitter's nano-environment. The differences in the Stokes shift are further supported by our observation that the excitation peak energies show a smaller distribution than the emission peak energies. Clearly, spectral variations in the emission spectra and the often observed spectral diffusion originate on the one hand from differences in the energy gap between the ground state and the excited state, and on the other hand from differences in the Stokes shift. We anticipate that that our results hold for all matrix-embedded single molecules, including fluorescent proteins for which the protein scaffold forms the embedding matrix. Finally, we believe that detailed characterization of single molecule Stokes shifts may be a promising tool to study local variations in a matrix, for example, in polymers. 


\section{REFERENCES}

1. Blum, C., et al., Room temperature excitation spectroscopy of single quantum dots. Beilstein Journal of Nanotechnology, 2011. 2: p. 516-524.

2. Lu, H.P. and X.S. Xie, Single-molecule spectral fluctuations at room temperature. Nature, 1997. 385(6612): p. 143-146.

3. Cotlet, M., et al., Identification of different emitting species in the red fluorescent protein dsred by means of ensemble and single-molecule spectroscopy. Proceedings of the National Academy of Sciences of the United States of America, 2001. 98(25): p. 14398-14403.

4. Schleifenbaum, F., et al., Single-molecule spectral dynamics at room temperature. Molecular Physics, 2009. 107(18): p. 1923-1942.

5. Wustholz, K.L., et al., Memory and spectral diffusion in single-molecule emission $\%$. Journal of Physical Chemistry C, 2008. 112(21): p. 7877-7885.

6. Blum, C., et al., Discrimination and interpretation of spectral phenomena by roomtemperature single-molecule spectroscopy. Journal of Physical Chemistry A, 2001. 105(29): p. 6983-6990.

7. Tian, Y.X., et al., Spectral diffusion of single dibenzoterrylene molecules in 2,3dimethylanthracene. Chemphyschem, 2012. 13(15): p. 3510-3515.

8. Hildner, R., et al., Single-molecule spectroscopy on a ladder-type conjugated polymer: Electron-phonon coupling and spectral diffusion. Chemphyschem, 2009. 10(14): p. 2524-2534.

9. Stracke, F., et al., Correlation of emission intensity and spectral diffusion in room temperature single-molecule spectroscopy. Chemphyschem, 2005. 6(7): p. 12421246.

10. Araoz, B., et al., Cage effect in poly(alkyl methacrylate) thin films studied by nile red single molecule fluorescence spectroscopy. Journal of Physical Chemistry C, 2012. 116(13): p. 7573-7580.

11. Blum, C. and V. Subramaniam, Single-molecule spectroscopy of fluorescent proteins. Analytical and Bioanalytical Chemistry, 2009. 393(2): p. 527-541.

12. Blum, C., A.J. Meixner, and V. Subramaniam, Single oligomer spectra probe chromophore nanoenvironments of tetrameric fluorescent proteins. Journal of the American Chemical Society, 2006. 128(26): p. 8664-8670.

13. Tinnefeld, P., D.-P. Herten, and M. Sauer, Photophysical dynamics of single molecules studied by spectrally-resolved fluorescence lifetime imaging microscopy (sflim). Journal of Physical Chemistry A, 2001. 105(34): p. 7989-8003.

14. Lakowicz, J.R., Principles of fluorescence spectroscopy. 3rd ed. 2006.

15. Mataga, N., H. Chosrowjan, and S. Taniguchi, Ultrafast charge transfer in excited electronic states and investigations into fundamental problems of exciplex chemistry: Our early studies and recent developments. Journal of Photochemistry and Photobiology C-Photochemistry Reviews, 2005. 6(1): p. 37-79.

16. Nishiyama, K., et al., Solvent dependence of stokes shift for organic solute-solvent systems: A comparative study by spectroscopy and reference interaction-site modelself-consistent-field theory. Journal of Chemical Physics, 2013. 139(9): p. 11.

17. Haidekker, M.A., et al., Effects of solvent polarity and solvent viscosity on the fluorescent properties of molecular rotors and related probes. Bioorganic Chemistry, 2005. 33(6): p. 415-425. 
18. Cotlet, M., et al., Identification of different emitting species in the red fluorescent protein dsred by means of ensemble and single-molecule spectroscopy. Proceedings of the National Academy of Sciences, 2001. 98(25): p. 14398-14403.

19. Krause, S., et al., Spectral diffusion of single molecules in a hierarchical energy landscape. Chemphyschem, 2011. 12(2): p. 303-312.

20. Araoz, B., et al., Cage effect in poly (alkyl methacrylate) thin films studied by nile red single molecule fluorescence spectroscopy. The Journal of Physical Chemistry C, 2012. 116(13): p. 7573-7580.

21. Blum, C., et al., Single molecule fluorescence spectroscopy of mutants of the discosoma red fluorescent protein dsred. Chem. Phys. Lett., 2002. 362(5-6): p. 355361.

22. Valeur, B. and M.N. Berberan-Santos, Molecular fluorescence - principles and applications. 2nd ed. 2013: Wiley-VCH.

23. Hinze, G., et al., Intramolecular electronic excitation energy transfer in donoracceptor dyads studied by time and frequency resolved single molecule spectroscopy. Journal of Chemical Physics, 2008. 128(12).

24. Royston, J.P., An extension of shapiro and wilk's $w$ test for normality to large samples. J. R. Stat. Soc. C, 1982. 31(2): p. 115-124.

25. Fisher, R.A., Statistical methods for research workers. 1925.

26. Efron, B., Nonparametric estimates of standard error: The jackknife, the bootstrap and other methods. Biometrika, 1981. 68(3): p. 589-599. 




\section{Chapter 5}

\section{Blinking statistics of colloidal quantum dots at different excitation wavelengths}

In imaging applications the blinking of quantum dots is an ambiguous phenomenon. Although unwanted in conventional imaging, blinking is of significant relevance for super-resolution microscopy. Controlling blinking characteristics allows for optimization for both conventional and super-resolution microscopy. We found indications for excitation wavelength-dependent quantum dot blinking in chapter 3. Some reports in literature support excitation wavelength-dependent blinking, while yet others contradict these observations. To investigate this disagreement we have systematically studied the effect of the excitation wavelength on blinking of $\mathrm{CdSe} / \mathrm{ZnS}$ core-shell quantum dots, commonly used in imaging applications, at 14 different excitation wavelengths across a spectral range from $535 \mathrm{~nm}$ to $620 \mathrm{~nm}$.

This chapter has been published in RSC Advances, 2013, 3, 17440-17445, Blinking statistics of colloidal quantum dots at different excitation wavelengths, DOI: $10.1039 /$ C3RA43637C 


\subsection{Blinking in modern fluorescence microscopy}

The role of blinking (see chapter 1) in imaging applications is ambiguous. In conventional imaging, blinking is a detrimental effect as it lowers the effective fluorescence quantum efficiency and label concentration, which in turn lengthens acquisition time. In single molecule tracking experiments blinking is unwanted, as it results in gaps in the trajectories when the emitters are in the dark state. However, in single molecule localization based super-resolution microscopy, blinking or state switching of fluorophores is at the heart of imaging structures beyond the diffraction limit, since it allows for the sequential localization of multiple emitters within one diffraction-limited spot [1-5].

Clearly, controlling blinking is interesting for both super-resolution and conventional microscopy, by allowing one to tune the blinking behavior to the desired application. A relatively simple approach to modulating blinking of organic fluorophores is by the addition of chemical additives that result in the formation of metastable dark states of the fluorophores. Blinking control of organic dyes has quickly become a much-used tool in super-resolution microscopy [5, 6].

Interestingly, our data in chapter 3 showed clues that point towards an increased probability for a transition to a dark state at specific excitation wavelengths, after continuous illumination with different excitation wavelengths [7], which is supported by recent reports which indicated that the blinking behavior of quantum dots depends on excitation wavelength [8, 9]. It has been suggested that these changes in blinking behavior with excitation wavelength originate from non-emissive trap states that can only be accessed by higher photon excitation energies [8, 9]. However, the changes in blinking characteristics with the excitation wavelength are not supported by other reports, where no clear relation between blinking and excitation wavelength has been observed for the visible range, while minor effects were observed for an excitation wavelength of $405 \mathrm{~nm}$ [10].

Using different excitation wavelengths to tune blinking would on the one hand allow for purely external control over blinking, without need for additives or sample modifications. On the other hand it would have implications for conventional imaging by allowing one to avoid excitation wavelengths that may lead to increased blinking. To settle the debate on excitation-wavelength dependent blinking characteristics, one needs to sample blinking statistics on a spectrally wide series of excitation wavelengths, which is an experimentally demanding challenge. Nevertheless, our instrumentation enables such a study because it has the flexibility to tune the excitation wavelength freely across the visible range (described in chapter 2), without requiring a large set of various laser sources. 


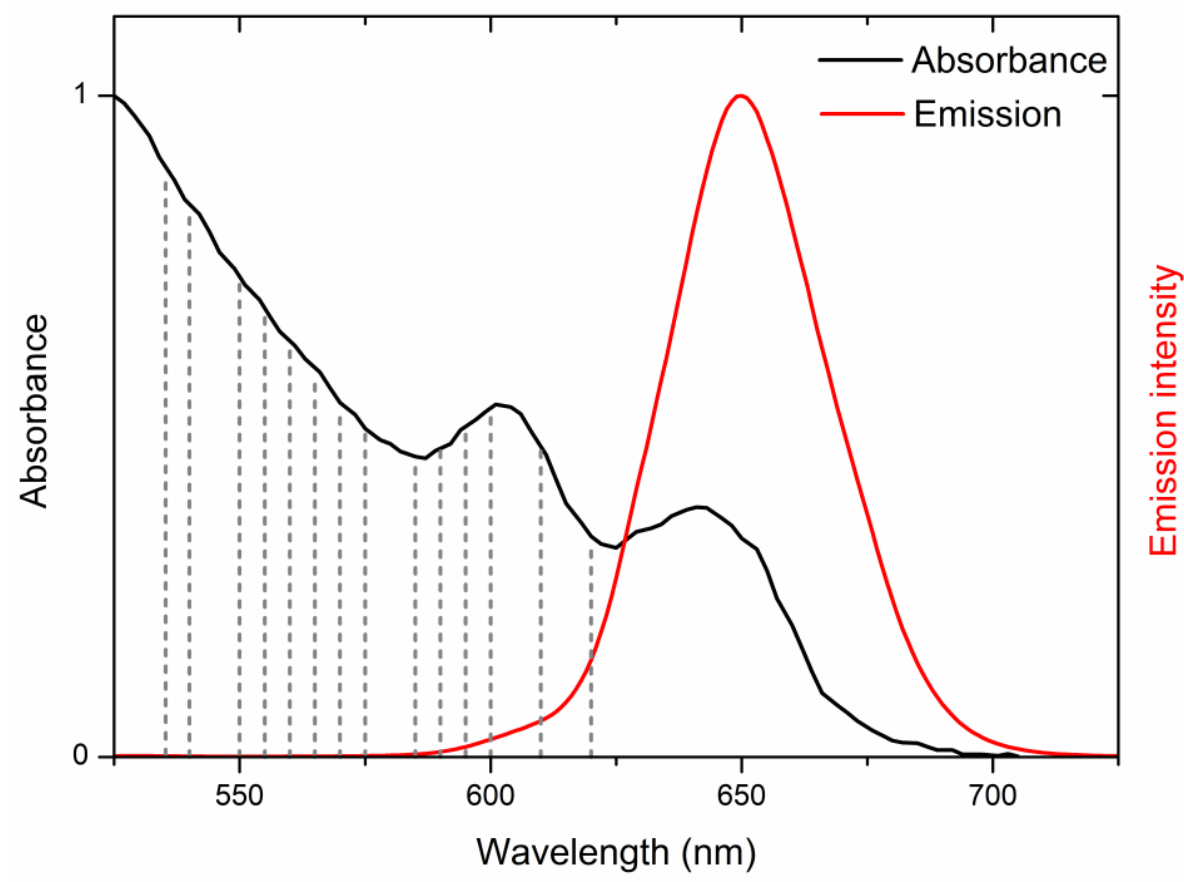

Figure 5.1 Normalized fluorescence absorbance and emission spectra of a solution of eFluor650 quantum dots. The dashed lines indicate the excitation wavelengths used in this study: $535,540,550,555,560,565,570,575,585,590,595,600,610,620 \mathrm{~nm}$

In this chapter, we set out to systematically study the blinking of commonly used quantum dots at different excitation wavelengths. We analyzed quantum dot blinking at 14 different fixed excitation wavelengths, ranging from $535 \mathrm{~nm}$ to $620 \mathrm{~nm}$ (Figure 5.1). This wide range of excitation wavelengths covers absorbance regions from the quasi-continuum to close to the band-gap energy of the quantum dots and thus includes excitation energies relative to the band gap that are comparable to those in earlier studies.

\subsection{Quantification of blinking}

To study the blinking of single emitters it is necessary to determine accurately when the emitter is in an emitting and when in a non-emitting state. The most common method to determine the times that an emitter resides in a bright, emitting state (sojourn times) is based on an intensity threshold for the identification of states [1012]. However, the outcome of the intensity threshold method has been shown to depend significantly on the choice of binning time and threshold level for quantum dots [13]. One of the reasons is that quantum dot blinking traces exhibit not only a 
bright, strongly emitting state, but also weakly emitting states and time-averaged intensity levels between states [14-16]. In the next chapter, we study the nature of these states in detail. To reduce the artifacts that arise due to the thresholding in intensity, a method has been introduced that uses not only the emission intensity, but also the lifetime within a time bin $[17,18]$. Here we use this method to identify the bright, strongest emitting state of $\mathrm{CdSe} / \mathrm{ZnS}$ quantum dots, based on the observation that the bright state is characterized by a typical emission lifetime around $20 \mathrm{~ns}$, while the weakly emitting states have a significantly lower lifetime [14-16]. The average photon arrival time $\left(\left\langle t_{a}\right\rangle\right.$, explained in more detail in the next chapter) reflects differences in decay dynamics between these states. Using both the intensity combined with the average photon arrival time (as an additional discrimination parameter (Figure 5.2)) [19] enhances the contrast between states and provide an additional handle to identify bright states in a fluorescence intensity-decay plot (FLID-plot, Figure 5.2b). The performances of the threshold and FLID discrimination methods are compared in the next section (5.3).

To identify time-bins belonging to the bright state and weakly emitting states we plot the intensity versus the average photon arrival time for each time bin (FLID plot, see Figure 5.2b). We use a $k$-means cluster analysis [20] to partition all timebins into two clusters in the FLID-plot. This method does not require a prior

a)

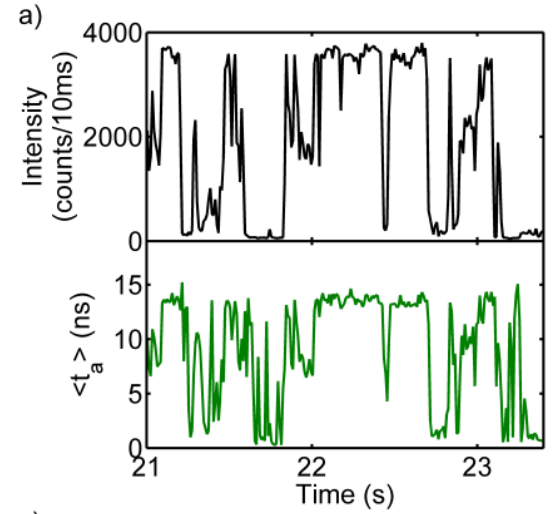

c)

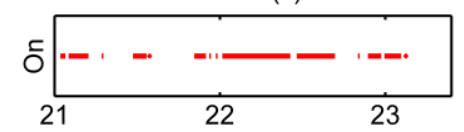

b)

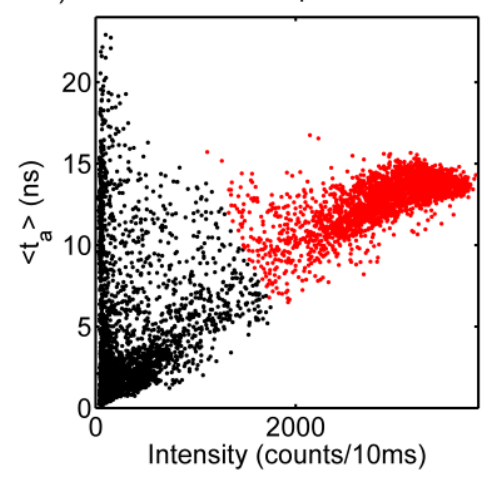

Figure 5.2 Cut-out of a time-trace (a) The time-bin size for both the intensity and the average photon arrival time trace is $10 \mathrm{~ms}$. (b) Plotted intensity versus average photon arrival time for all time-bins of the recorded time-trace. Two groups are identified, time bins either belong to the bright state (red) or to weakly emitting states (black). Based on this identification, the sojourn times are determined (see c), bottom panel). 
defined threshold, but iteratively splits the experimentally recorded data set in two clusters, the on-state and all other weakly emitting states, until these two clusters converge to a maximal statistical separation. Also in this method the discrimination between the clusters follows a curved path in the FLID plot, rather than a more limited hard boundary in intensity and average lifetime. The time-bins that correspond to the bright state ('on') are marked (Figure 5.2b, red points). The sojourn times are calculated from the number of connected time-bins of the bright state using a binary mask (Figure 5.2c). These sojourn times are summarized in a probability density histogram which is calculated by the formalism proposed by Kuno et al. [21]

$$
P_{d}\left(t_{o n}\right)=\frac{N\left(t_{o n}\right)}{\sum N\left(t_{o n}\right)} \cdot \frac{1}{\delta t_{o n}}
$$

where $N\left(t_{o n}\right)$ is the number of events at a defined range $\delta t_{o n}$ around $t_{o n}$. For quantum dots, the probability density histogram typically shows a linear behavior on $\log -\log$ scale, with a typical slope ranging between -1.2 and -1.7 [22-24], and slight bending at long sojourn times.

\subsection{Evaluation of blinking analysis methods}

The probability density histograms generated when using an intensity only based method [13] have been shown to be very sensitive to the choice of bin time and threshold used. We tested the performance of both the intensity threshold method and the FLID discrimination method used here using simulated time-traces of 30 second lengths (Figure 5.3ab). In these simulated traces we assume for simplicity two states, a bright, strongly emitting state of long lifetime, and one weakly emitting state of shorter lifetime. The sojourn times are randomly distributed following a power-law with a coefficient of -1.6 , which we typically find for our quantum dot samples. The shortest sojourn times that are simulated have a period of $0.1 \mathrm{~ms}$ to allow for state fluctuations that are faster than the binning time of $10 \mathrm{~ms}$.

As a first test we used an average intensity of 500 counts per $10 \mathrm{~ms}$ and lifetime of $20 \mathrm{~ns}$ for the bright state, and a dim state of average intensity of 100 counts per $10 \mathrm{~ms}$ and $5 \mathrm{~ns}$ lifetime as observed before [14, 15] (Figure 5.3ac). We determined the sojourn times of the simulated dataset using both the intensity threshold and the FLID discrimination methods. The statistics of these sojourn times are determined via eq. 5.1 and the resulting probability density diagrams are shown in Figure 5.3c. 
a)

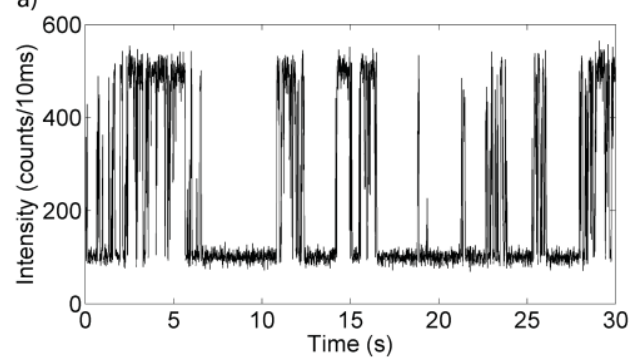

c)

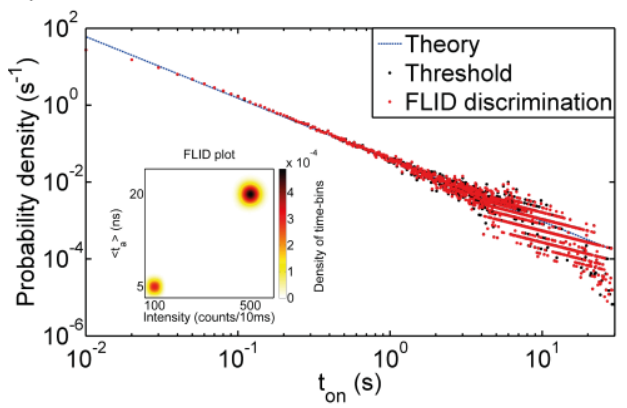

b)

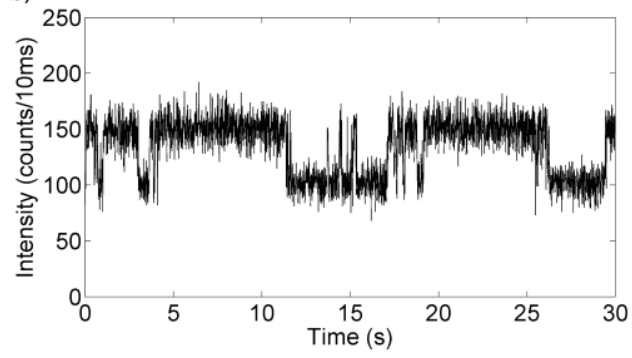

d)

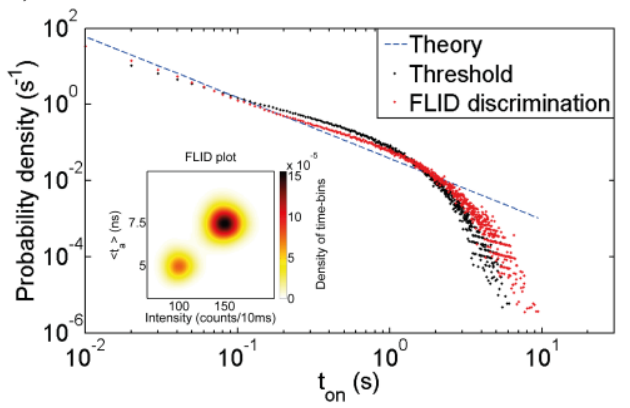

Figure 5.3 Simulated quantum dot blinking traces by a simple two-state blinking model, where the sojourn times are randomly distributed by a power-law with an exponent of -1.6. Case (a): Bright state (intensity 500 counts/s, lifetime $20 \mathrm{~ns}$ ); weakly emitting state (intensity 100 counts/s, lifetime 5 ns). Case (b): Bright state (intensity 150 counts/s, lifetime $7.5 \mathrm{~ns}$ ); weakly emitting state (intensity 100 counts/s, lifetime $5 \mathrm{~ns}$ ). The probability density histograms of the simulated traces are shown in (c) for case (a) and in (d) for case (b). The insets indicate the intensity and decay separation between the two states.

As can be expected, we find no clear differences in the performance of both methods when the emission of the two states is clearly distinguished. Both methods show very close resemblance to the theoretically expected probability density curve. We observe bending at long sojourn times in Figure 5.3c, that can be assigned to the simulated total time window length of 30 seconds per trace, which is the principal upper limit for the sojourn times. Further, the probability density curves show some underestimation at short sojourn times. This is an effect of the high probability to have sojourn times that are shorter than the binning time leading to a high probability to miss state jumps to weakly emitting states. Missed state transitions to weakly emitting states result in an overestimation of long sojourn times. Note that this leads to an underestimation of the steepness of the slope, which is a parameter that is generally used to characterize and quantify blinking statistics.

We then evaluated the performance of both methods for two states that are not clearly separated but have overlapping distributions as is commonly observed in experimentally recorded single emitter intensity traces $[8,11]$. We bring the intensity 
and decay values of bright and weakly emitting states closer to each other to find a configuration where both methods start to noticeably deviate from the expected result. In Figure 5.3bd we show the result of our simulation using a bright state intensity of 150 counts $/ 10 \mathrm{~ms}$ and lifetime of $7.5 \mathrm{~ns}$ and weakly emitting state intensity of 100 counts $/ 10 \mathrm{~ms}$ and lifetime of $5 \mathrm{~ns}$. Using these parameters both methods show a deviation from the expected result. However, the inclusion of the additional parameter average photon arrival time in the FLID method yields a curve that more closely approaches the theoretical values than the intensity threshold method. Note that the plot is on a double logarithmic scale. The deviation of both methods from the expected values arises from the overlap of the statistical distributions of both the intensity and the average photon arrival time of the two states. This effect can be seen in the overlap between the two states in the FLID density plot. On the one hand this leads to more short false positives, which shift the probability density curve towards short sojourn times, while on the other hand the decreased intensity contrast leads to an increased underestimation of the probability of short on times.

In our analysis we aim at detecting modifications in blinking behavior by monitoring changes of the probability density histogram. Our simulations show that the shape and bending of the probability curve are insufficiently robust when the state discrimination is not clear. We therefore chose to directly calculate the probability to find short sojourn times and long sojourn times by integrating the area beneath the probability density curve. In this way, the probabilities determined do not depend on any fitting model and are a direct read-out from the data.

\subsection{Experiment}

At 14 different excitation wavelengths, we recorded 30 seconds long photon streams for each quantum dot (Table 5.1). The instrumentation is similar to that described in chapter 2, except that a Semrock 633SP filter is located at the excitation light path, which constraints the spectral excitation range and suppresses unwanted excitation light. Also, in the detection branch of the microscope a Semrock 633LP and a Semrock $675 / 67 \mathrm{BP}$ filter prevent scattered or reflected excitation light leaking through to the detector and also limit the detection range within the narrow emission

Table 5.1 The number of analyzed quantum dot time-traces per excitation wavelength

\begin{tabular}{|ccccccccccccccc|}
\hline$\lambda_{\text {exc }}(\boldsymbol{n m})$ & 535 & 540 & 550 & 555 & 560 & 565 & 570 & 575 & 585 & 590 & 595 & 600 & 610 & 620 \\
\hline $\boldsymbol{N}_{\text {dots }}$ & 57 & 37 & 24 & 12 & 636 & 37 & 53 & 57 & 37 & 46 & 49 & 22 & 87 & 35 \\
\hline
\end{tabular}


band of eFluor650 quantum dots. The samples were prepared according to the standard procedure described in chapter 2. All quantum dots studied were embedded in a film of PVA to avoid any influence of differences in the dielectric environment [25]. Since it has been shown that blinking statistics depends on excitation power, $[26,27]$ we adjusted the excitation power to yield equal emission intensity for the different excitation wavelengths. The excitation power that was used was $\sim 1 \mathrm{~kW} / \mathrm{cm}^{2}$, comparable to the excitation power typically used in other studies [28-32].

From the recorded photon streams, we calculated intensity and $\left\langle t_{a}\right\rangle$-traces using a binning time of 10 milliseconds, in agreement with the requirements for objective blinking analyses developed by Crouch et al. [13] We analyzed all traces that showed a highest intensity cluster above an intensity of 250 counts $/ 10 \mathrm{~ms}$ and an average photon arrival time above $10 \mathrm{~ns}$ in the FLID plots to avoid biasing, e.g. by only weakly emitting state traces. We quantified blinking as described above using the FLID discrimination method. We then constructed one probability density

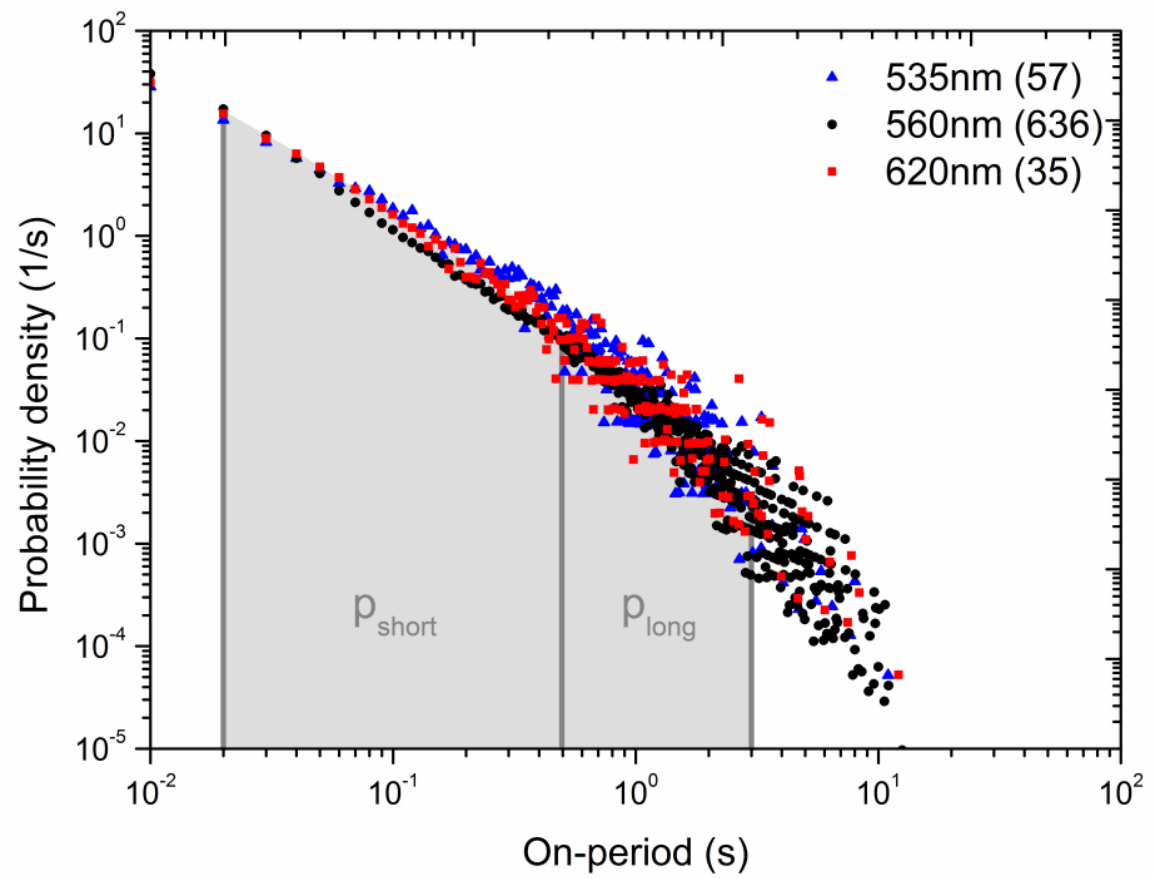

Figure 5.4 Probability density diagrams of bright state sojourn times at three different excitation wavelengths ranging from far above to close to the band-gap energy. The numbers in between parentheses indicate the number of quantum dots that are used for the blinking statistics. 
histogram from all sampled quantum dots per excitation wavelengths, according to eq. 5.1. Because the probability density from a single quantum dot measurement already follows a power law [21, 22, 33] we compiled the data from many quantum dots in the blinking analysis to add statistical relevance [10, 26]. In Figure 5.4 we present representative probability density diagrams of three excitation wavelengths that range from far above to close to the band-gap energy. Indeed we find the known clear signature of a power law distribution of the sojourn times for all excitation wavelengths, irrespective of the number of sampled quantum dots per excitation wavelength. The sojourn times range over two decades of time and three decades of probability. For all excitation wavelengths, we find a slope of about -1.6, which corresponds well with the power-law coefficients found in literature ranging between -1.2 and -1.7 [22-24]. All probability density curves at the 14 different excitation wavelengths are strongly overlapping and show no clear differences.

Changes in the blinking behavior result in changed probabilities to find long or short lasting sojourn times. To quantify the blinking characteristics for different excitation wavelengths we directly determined the probability to find short sojourn times. To meet the Nyquist theorem, we do not consider the $10 \mathrm{~ms}$ sojourn times, which is the time-resolution of our measurements. On the other extreme, the recording window length limits the detection of long sojourn times. Since long sojourn times scarcely occur, we also neglect sojourn times that are longer than 3 seconds. We directly calculate the probability to find short sojourn times between $20 \mathrm{~ms}$ and $0.5 \mathrm{~s}\left(p_{\text {short }}\right)$ and probability to find long sojourn times between $0.5 \mathrm{~s}$ and $3 \mathrm{~s}\left(p_{\text {long }}\right)$, by integrating the area beneath the probability density curve (Figure 5.4). The probability values are obtained from the averaged results of non-parametric statistical bootstrapping [34] of the probability density curves. In statistical bootstrapping, a measured set of sojourn times is randomly sampled with replacement until the sample population is equally large as the measured set of sojourn times. Statistics of a thousand randomly sampled populations are used to obtain 95\% confidence bounds and average probabilities to find short or long sojourn times. The $95 \%$ confidence bounds indicate the reliability of the probability values that are obtained from the existing population of quantum dots. However, it should be noted that these confidence bounds do not reflect the reproducibility of the parameter values on a different set of quantum dots, meaning that this confidence interval underestimates the true error of the measurement. The result of this analysis is shown in Figure 5.5.

We observe that variations in the probabilities to find short $\left(p_{\text {short }}\right)$ and long $\left(p_{\text {long }}\right)$ sojourn times for the different excitation wavelengths are between 0.40 to 


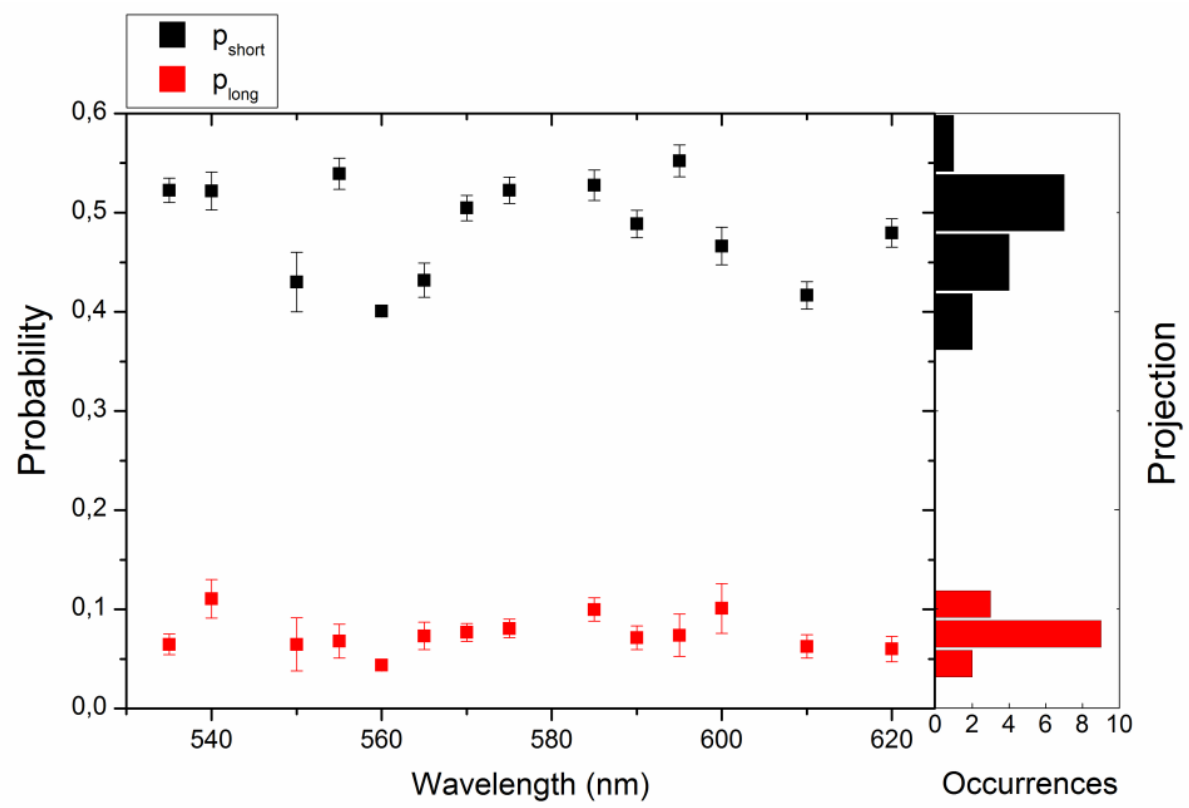

Figure 5.5 Probabilities to find sojourn times between $20 \mathrm{~ms}$ and $0.5 \mathrm{~s}$ ( $p_{\text {stort }}$, black) and between $0.5 \mathrm{~s}$ and $3 \mathrm{~s}\left(p_{\mathrm{om} n}, \mathrm{red}\right)$, for 14 different excitation wavelengths. The probability to find long or short sojourn times varies and shows distributions around $0.49 \pm 0.05$ and $0.075 \pm 0.018$.

0.55 and 0.05 to 0.11 , respectively. The probability to find longer sojourn times shows a larger relative variation, which is likely to arise from the fact that the chance to find these long sojourn times is generally low, resulting in a higher uncertainty of the measurement. The observed differences do not follow any recognizable trend, or exhibit any features that may be attributed to specific sections of the absorbance spectrum, such as the excitonic peak or the quasi-continuum region. We observe no prominent changes for excitation wavelengths ranging from far above to close to the band-gap energy.

Our finding that the excitation wavelength has no major effect on quantum dot blinking agrees with earlier results in which no effect of different excitation wavelength in the visible on quantum dot blinking has been observed [10]. At the blinking times up to 3 seconds sampled in our study we do not find the distinct differences in quantum dot blinking reported earlier. The statistical relevance and validity of the systematical study described in this chapter is superior to the primary blinking analysis described in chapter 3 . In addition, in chapter 3 , as well as in the other studies that report on changes in blinking as a function of the excitation wavelengths [7-9], these changes have been observed primarily for prolonged illumination and longer timescales outside the range sampled by us in the current 
study. Due to the power law distribution of the on time durations, on times longer than the 3 seconds occur so rarely that influencing this long time blinking has no practical implications on imaging.

\subsection{Conclusion}

The small variations in blinking that we observe show no trend with excitation wavelengths or any features that may be attributed to specific sections of the absorbance spectrum, such as the excitonic peak or the quasi-continuum region. The small variations observed do not allow for a decided and predictive increase or decrease of blinking. Based on these results, a change of the blinking of quantum dots with excitation wavelength is too small to be used effectively to control blinking in imaging applications and thus does not have to be considered when using quantum dots as luminescent markers.

\section{REFERENCES}

1. Lidke, K.A., et al., Superresolution by localization of quantum dots using blinking statistics. Optics Express, 2005. 13(18): p. 7052-7062.

2. Ha, T. and P. Tinnefeld, Photophysics of fluorescent probes for single-molecule biophysics and super-resolution imaging, in Annual review of physical chemistry, vol 63, M.A. Johnson and T.J. Martinez, Editors. 2012, Annual Reviews: Palo Alto. p. 595-617.

3. Bates, M., et al., Multicolor super-resolution imaging with photo-switchable fluorescent probes. Science, 2007. 317(5845): p. 1749-1753.

4. Heilemann, M., et al., Subdiffraction-resolution fluorescence imaging with conventional fluorescent probes. Angewandte Chemie International Edition, 2008. 47(33): p. 6172-6176.

5. Vogelsang, J., et al., Make them blink: Probes for super-resolution microscopy. Chemphyschem, 2010. 11(12): p. 2475-2490.

6. Heilemann, M., et al., Super-resolution imaging with small organic fluorophores. Angewandte Chemie International Edition, 2009. 48(37): p. 6903-6908.

7. Blum, C., et al., Room temperature excitation spectroscopy of single quantum dots. Beilstein Journal of Nanotechnology, 2011. 2: p. 516-524.

8. Knappenberger, K.L., et al., Excitation wavelength dependence of fluorescence intermittency in cdse/zns core/shell quantum' dots. Nano Letters, 2007. 7(12): p. 3869-3874.

9. Knappenberger, K.L., et al., Excitation-wavelength dependence of fluorescence intermittency in cdse nanorods. Acs Nano, 2008. 2(10): p. 2143-2153.

10. Crouch, C.H., et al., Excitation energy dependence of fluorescence intermittency in cdse/zns core-shell nanocrystals. Journal of Physical Chemistry C, 2009. 113(28): p. 12059-12066. 
11. Ma, X., et al., Optical spectroscopy of single semiconductor nanocrystals close to $g$ old nanoparticles. Colloidal Nanocrystals for Biomedical Applications VII, 2012: p. 82321I-82321I.

12. Ratchford, D., et al., Photoluminescence dynamics of ensemble and individual cdse/zns quantum dots with an alloyed core/shell interface. Journal of Applied Physics, 2011. 109(10).

13. Crouch, C.H., et al., Facts and artifacts in the blinking statistics of semiconductor nanocrystals. Nano Letters, 2010. 10(5): p. 1692-1698.

14. Schlegel, G., et al., Fluorescence decay time of single semiconductor nanocrystals. Phys Rev Lett, 2002. 88(13): p. 137401.

15. Fisher, B.R., et al., Emission intensity dependence and single-exponential behavior in single colloidal quantum dot fluorescence lifetimes. Journal of Physical Chemistry B, 2004. 108(1): p. 143-148.

16. Gomez, D.E., et al., Exciton-trion transitions in single cdse-cds core-shell nanocrystals. Acs Nano, 2009. 3(8): p. 2281-2287.

17. Amecke, N. and F. Cichos, Intermediate intensity levels during the emission intermittency of single cdse/zns quantum dots. Journal of Luminescence, 2011. 131(3): p. 375-378.

18. Galland, C., et al., Two types of luminescence blinking revealed by spectroelectrochemistry of single quantum dots. Nature, 2011. 479(7372): p. 203207.

19. Galland, C., et al., Lifetime blinking in nonblinking nanocrystal quantum dots. Nature communications, 2012. 3: p. 908.

20. Hartigan, J.A. and M.A. Wong, Algorithm as 136: A k-means clustering algorithm. J. R. Stat. Soc. C, 1979. 28(1): p. 100-108.

21. Kuno, M., et al., Nonexponential "blinking" kinetics of single cdse quantum dots: A universal power law behavior. Journal of Chemical Physics, 2000. 112(7): p. 31173120.

22. Bharadwaj, P. and L. Novotny, Robustness of quantum dot power-law blinking. Nano Letters, 2011. 11(5): p. 2137-2141.

23. Frantsuzov, P., et al., Universal emission intermittency in quantum dots, nanorods and nanowires. Nature Physics, 2008. 4(7): p. 519-522.

24. Malko, A.V., et al., Pump-intensity- and shell-thickness-dependent evolution of photoluminescence blinking in individual core/shell cdse/cds nanocrystals. Nano Letters, 2011. 11(12): p. 5213-5218.

25. Issac, A., et al., Influence of the dielectric environment on the photoluminescence intermittency of cdse quantum dots. Chemphyschem, 2012. 13(13): p. 3223-3230.

26. Cordones, A.A., T.J. Bixby, and S.R. Leone, Evidence for multiple trapping mechanisms in single cdse/zns quantum dots from fluorescence intermittency measurements over a wide range of excitation intensities. Journal of Physical Chemistry C, 2011. 115(14): p. 6341-6349.

27. Goushi, K., T. Yamada, and A. Otomo, Excitation intensity dependence of powerlaw blinking statistics in nanocrystal quantum dots. Journal of Physical Chemistry C, 2009. 113(47): p. 20161-20168.

28. Zhao, J., et al., Challenge to the charging model of semiconductor-nanocrystal fluorescence intermittency from off-state quantum yields and multiexciton blinking. Physical Review Letters, 2010. 104(15). 
29. Schmidt, R., C. Krasselt, and C. von Borczyskowski, Change point analysis of matrix dependent photoluminescence intermittency of single cdse/zns quantum dots with intermediate intensity levels. Chemical Physics, 2012(0).

30. Cordones, A.A., T.J. Bixby, and S.R. Leone, Direct measurement of off-state $t$ rapping rate fluctuations in single quantum dot fluorescence. Nano Letters, 2011. 11(8): p. 3366-3369.

31. Schwartz, O., et al., Colloidal quantum dots as saturable fluorophores. Acs Nano, 2012.

32. Fisher, B., et al., Multiexciton fluorescence from semiconductor nanocrystals. Chemical Physics, 2005. 318(1-2): p. 71-81.

33. Cichos, F., C. von Borczyskowski, and M. Orrit, Power-law intermittency of single emitters. Current Opinion in Colloid \& Interface Science, 2007. 12(6): p. 272-284.

34. Efron, B., Nonparametric estimates of standard error: The jackknife, the bootstrap and other methods. Biometrika, 1981. 68(3): p. 589-599. 



\section{Chapter 6}

\section{Discrete distribution of grey states in single $\mathrm{CdSe} / \mathrm{ZnS}$ core-shell quantum dots}

In chapter 5 we studied the effect of the excitation wavelength on blinking behavior of single quantum dots. In this chapter we study the blinking mechanism of these quantum dots in detail by implementing and expanding the intensity-decay analysis technique used in the previous chapter. We identify a discrete distribution of grey states in addition to the earlier observed continuous distribution of grey states, and quantitatively measure the decay characteristics of these discrete states to learn more about the blinking mechanism. Our results lead us to propose a modification to the existing trapped charge model for quantum dot blinking to explain our observations in a manner consistent with earlier reports. 


\subsection{Quantum dot blinking}

In the previous chapter, the focus was mainly on the blinking behavior of the bright state. As already mentioned in the previous chapter, blinking is more complex in reality than a simple two state model describes. In this chapter, we implement the FLID method used in the previous chapter to study in more detail the mechanism behind the blinking, by considering the blinking behavior of the dim states as well. Before jumping to the experiments, it is important to first understand the extensive research history on quantum dot blinking [1-12].

Quantum dot blinking was first described by a simple two state blinking model (charging model), in which the 'on' state is an emissive state that radiatively relaxes, and the 'off' state occurs due to an Auger recombination [13, 14] which ejects an electron of an exciton producing a photoionized positively charged core which strongly quenches newly generated excitons and prevents radiative relaxation. The quantum dot starts emitting again as soon as an electron recombines with the hole that is left at the core of the quantum dot. However, this charging model predicts exponentially distributed blinking behavior for the 'on' states and power-law distributed behavior for the 'off' states. In practice, power-law distributed blinking behavior is observed for both 'on' and 'off' states. Verberk et al. [15] modified the charging model by additionally allowing hole trapping far away from the core, to explain the observation of the prolonged 'on' states, giving rise to power-law distributed blinking statistics for the 'on' states. Moreover, Verberk et al. mentioned that a broad distribution of luminescence levels may be expected because the trapped hole's Coulomb potential slowly varies with distance, and still effectively prevents ionization. Schlegel, Fisher, and Zhang [16-18] experimentally showed that besides the 'on'/'off' states, there exists a continuous distribution of intermediate emission intensities that are not time-averaged emission intensities of the 'on' and 'off' state. The intermediate emission intensities show multi-exponential decay characteristics at the nanosecond scale and show a strong linear correlation between fluorescence intensity and lifetime [18], having a long average lifetime for states with high intensity and short average lifetime for states with low intensity. This linear correlation is a typical signature of quenching and indicates that fluctuations in the non-radiative decay path are dominant. The decay fluctuations in time are explained via charge trapping states that vary with time and quench the emissive state (fluctuating trap model). Since charge carrier trapping at the surface of the core would produce decay characteristics at the sub-nanosecond scale, the trap states must be located further away from the core $[18,19]$. Shell surface and other external trap states near the quantum dot can capture charge carriers [20, 21], providing a source 
of non-radiative pathways for the newly generated excitons in quantum dots. The trapped charge carriers would produce nanosecond scale decay characteristics and allow for intermediate intensity levels, which depend on the distance between the core and the trapped charge carrier, giving more non-radiative (faster) decay and a dim intermediate intensity for a charge that is trapped closer to the core, and vice versa.

\subsection{Blinking analysis using FLID diagrams}

During the course of our study in the previous chapter, on the blinking statistics of quantum dots at different excitation wavelengths, we found strong indications that besides the continuum of intermediate states there also exist states that have a defined combination of intensity and decay time. In this chapter, we therefore study the fluorescence emission of the intermediate states of single $\mathrm{CdSe} / \mathrm{ZnS}$ core/shell colloidal quantum dots by monitoring the intensity and decay properties, and by examining the corresponding FLID diagrams at a time-scale that is in the order of $\sim 30-60$ s. Unlike others in literature, we use the average photon arrival time $\left(\left\langle t_{a}\right\rangle\right)$ rather than the exponentially fitted lifetime $(\tau)$ to monitor the evolution of decay over time. The average photon arrival time does not require fitting models, does not depend on the complexity of the decay characteristics, and is also a direct measure of the mean lifetime of all decay paths taken together (see section 6.3 below). The advantage of using the average photon arrival time is that the time resolution of the emission trace increases significantly compared to using the lifetime, because a small number of photons $(\sim 100)$ already gives an accurate estimate of the decay time, even for multi-exponential decay characteristics. The only disadvantage is that the average photon arrival time does not reveal the number of decay components, which requires exponential fitting analyses.

\subsection{Average photon arrival time $\left(\left\langle\boldsymbol{t}_{\boldsymbol{a}}\right\rangle\right)$}

In general, decay characteristics are described by a sum of $n$ independent exponential decay components:

$$
y(t)=\sum_{i=1}^{n} a_{i} \cdot e^{-t / \tau_{i}}
$$

Where $a_{i}$ is the amplitude of the exponential decay for component $i$, and has a corresponding lifetime $\tau_{i}$. The average photon arrival time $\left\langle t_{a}\right\rangle$ is the overall expectation value $(\mu)$ of the photon arrival times, which is derived by taking the first moment of the decay characteristics: 


$$
\left\langle t_{a}\right\rangle=\mu=\frac{\int_{0}^{\infty} y(t) \cdot t d t}{\int_{0}^{\infty} y(t) d t}
$$

For a sum of independent exponential decay components, and using integration by parts, this equation reduces to:

$$
\left\langle t_{a}\right\rangle=\frac{\sum_{i=1}^{n}\left[-a_{i} \tau_{i} \cdot e^{-t} / \tau_{i}\right]_{0}^{\infty}+a_{i} \tau_{i} \int_{0}^{\infty} e^{-t / \tau_{i}} d t}{\sum_{i=1}^{n} \int_{0}^{\infty} a_{i} \cdot e^{-t / \tau_{i}} d t}=\frac{\sum_{i=1}^{n} a_{i} \tau_{i}^{2}}{\sum_{i=1}^{n} a_{i} \tau_{i}} \equiv \bar{\tau}
$$

showing that the average photon arrival time $\left(\left\langle t_{a}\right\rangle\right)$ is equal to the mean lifetime $(\bar{\tau})$ [22]. However, to obtain the average photon arrival time one simply calculates the average of the photon arrival times of the detected photons, which can be done using any number of detected photons, unlike the exponential fitting algorithms, which require several orders of magnitude more photons for proper fitting of the decay characteristics, especially for multi-exponential decay characteristics. Alternative approaches to multi-exponential decay fitting, that deal with a similar amount of photons as the $\left\langle t_{a}\right\rangle$-method, require a priori characterization of the individual decay components [23]. The average photon arrival time thus allows for a good approximation of the mean lifetime, and is particularly useful for our application because the emission traces of single emitters typically exhibit a low number of photons per time-bin. Therefore, the average photon arrival time gives access to monitoring of the mean lifetime of the individual states of single emitters, at the millisecond time-scale.

Furthermore, considering that in general the number of detected photons is equal to the full integral over the decay characteristics, it can be derived that for each exponential decay component $i$, the number of collected photons $N_{p h, i}$ is equal to the product of the amplitude $a_{i}$ and lifetime $\tau_{i}$ :

$$
\begin{gathered}
\int_{0}^{\infty} y_{i}(t) d t=N_{p h, i} \\
\int_{0}^{\infty} a_{i} \cdot e^{-t / \tau_{i}} d t=a_{i} \cdot \tau_{i}=N_{p h, i}
\end{gathered}
$$

which simplifies equation (6.3) to: 


$$
\begin{gathered}
\left\langle t_{a}\right\rangle=\bar{\tau}=\sum_{i=1}^{n} f_{i} \cdot \tau_{i} \\
f_{i}=\frac{N_{\text {phot }, i}}{\sum_{i=1}^{n} N_{\text {phot }, i}}\left(=\frac{a_{i} \cdot \tau_{i}}{\sum_{i=1}^{n} a_{i} \cdot \tau_{i}}\right)
\end{gathered}
$$

where $f_{i}$ is the fraction of decay component $i$, showing that the average photon arrival time is the intensity weighted average of the lifetimes over all decay components.

Generally, following the same line of argumentation, it can be derived that the overall expectation value of any distribution being a sum of linearly independent distributions is the fractional weighted sum of the expectation values of the separate distributions.

$$
\begin{aligned}
\mu & =\frac{\int_{0}^{\infty} y(t) \cdot t d t}{\int_{0}^{\infty} y(t) d t} \\
& =\frac{1}{\int_{0}^{\infty} y(t) d t} \int_{0}^{\infty}\left(y_{1}(t)+y_{2}(t)+\cdots\right) \cdot t d t \\
& =\frac{1}{\int_{0}^{\infty} y(t) d t}\left[\int_{0}^{\infty} y_{1}(t) \cdot t d t+\int_{0}^{\infty} y_{2}(t) \cdot t d t+\cdots\right] \\
\mu & =f_{1} \cdot \mu_{1}+f_{2} \cdot \mu_{2}+\cdots
\end{aligned}
$$

The latter equation proves that the derived formulation for the expectation value is not restricted to solely exponential distributions, but is valid for any arbitrary distribution (including the background signal, which has an expectation value exactly the middle of the photon arrival time detection window, which is in our case $1 / 2 \cdot 50 n s=25 n s)$.

\subsection{Observation of definite states in single quantum dot emission traces}

After preparing the sample ( $\mathrm{CdSe} / \mathrm{ZnS}$ core/shell quantum dots, eFluor650), we locate the single quantum dots by raster scanning an area of $10 \mathrm{x} 10 \mu \mathrm{m}^{2}$ (for example see figure 2.6a), using the same instrumentation as used in the previous chapter. After identifying the single quantum dots, we illuminate the fully isolated emitters one-by-one and record the fluorescence emission over 30 seconds, using TCSPC (Figure 6.1ab). From the TCSPC photon stream (for more details, see chapter 2) we obtain absolute photon arrival times, of which the macrotimes are used to categorize 
photons into groups using a long sequence of equally spaced $10 \mathrm{~ms}$ time bins. Once categorized, we derive both the intensity (number of photons of each photon group) and the average photon arrival time (average microtime of each photon group) of each $10 \mathrm{~ms}$ time bin. This procedure allows us to follow both the intensity and average photon arrival time $\left(\left\langle t_{a}\right\rangle\right)$ synchronously over time, for a single quantum dot. Long $\left\langle t_{a}\right\rangle$ values reflect slow decay and short $\left\langle t_{a}\right\rangle$ a fast decay.

In Figure 6.1ab, we observe blinking between high, intermediate and low intensity levels and see correlated features for the average photon arrival time. High intensity levels show slow decay, whereas low intensity levels show fast decay, which is in agreement with literature [16-18, 24].

As already introduced in the previous chapter, another way of visualizing the correlation between intensity and decay is by so-called fluorescence lifetime intensity diagrams [17] (FLID map, Figure 6.1c). In addition to the scatterplot that was used in the previous chapter, the axes of the decay versus intensity plot are sectioned into a discrete set of 2D bins. These 2D bins are then used to make a histogram of the different combinations of intensity and decay. Therefore, the FLID maps used in this chapter highlight frequently occurring combinations of intensity and lifetime.

a)

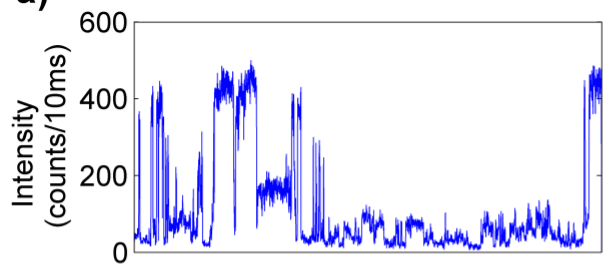

b)

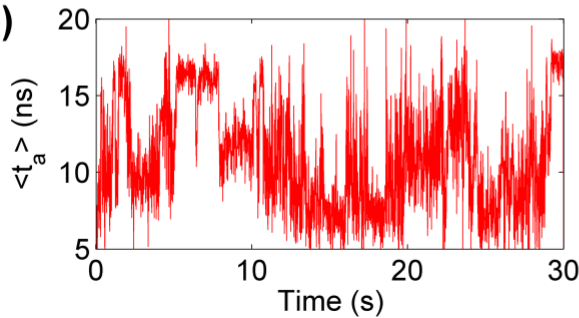

c)

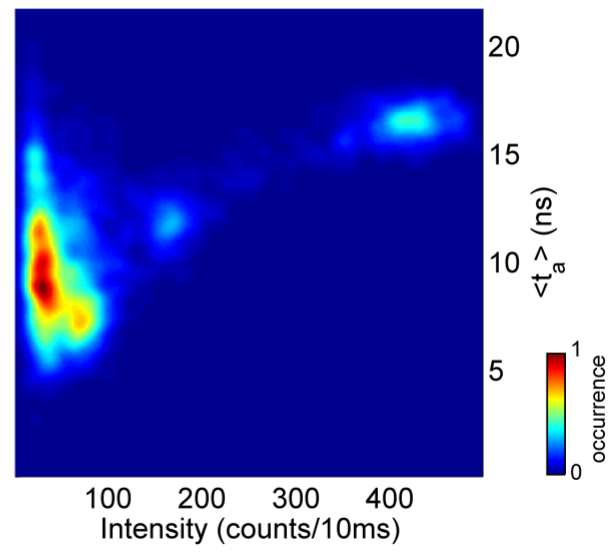

Figure 6.1 Example of fluorescence emission traces of a single $\mathrm{CdSe} / \mathrm{ZnS}$ core-shell quantum dot. a) the blue trace represents the evolution of intensity over time. b) the red trace represents the average photon arrival time $\left(\left\langle\mathrm{t}_{\mathrm{t}}\right\rangle\right)$ for each $10 \mathrm{~ms}$ time-point, which is representative of decay dynamics. (c) Intensity and $\left\langle\mathrm{t}_{\mathrm{a}}\right\rangle$ are correlated in time as can be seen when plotting intensity versus $\left\langle\mathrm{t}_{\mathrm{t}}\right\rangle$ in a FLID map, which visualizes frequently occurring combinations of intensity and $\langle\mathrm{t}\rangle\rangle$. 
The linear color-scale at the FLID map is normalized to the peak maximum of the 2D histogram.

Overall, we see a trend in the FLID map from a peak with high intensity and slow decay towards peaks with low intensity and fast decay (Figure 6.1c), similar to the generic behavior for the continuum of intermediate states [16-18, 24]. Remarkably, where we expected to find a continuum of intermediate states [16-18, 24, 25], which should appear as a diffuse smear from bright and slowly decaying time-points to dim and fast decaying time-points in the FLID, instead, we clearly observe multiple distinct peaks. In detail, we observe a bright peak around an intensity level of 430 counts per $10 \mathrm{~ms}$, having a $\left\langle t_{a}\right\rangle$ of $\sim 17 \mathrm{~ns}$, which is close to the typically expected lifetime for the brightest state $(\sim 20 \mathrm{~ns})[16,18]$. Furthermore, we observe peaks with faster decay around an intensity level of 170 counts per $10 \mathrm{~ms}$ and 80 counts per $10 \mathrm{~ms}$, which are also visibly present in the intensity trace. In addition, we see a smeared out group at the lowest intensity, which is broadened because of the increasing inaccuracy in $\left\langle t_{a}\right\rangle$ due to the strong influence of the background signal (as expected from section 6.3). The $\left\langle t_{a}\right\rangle$ is the weighted average of all components in

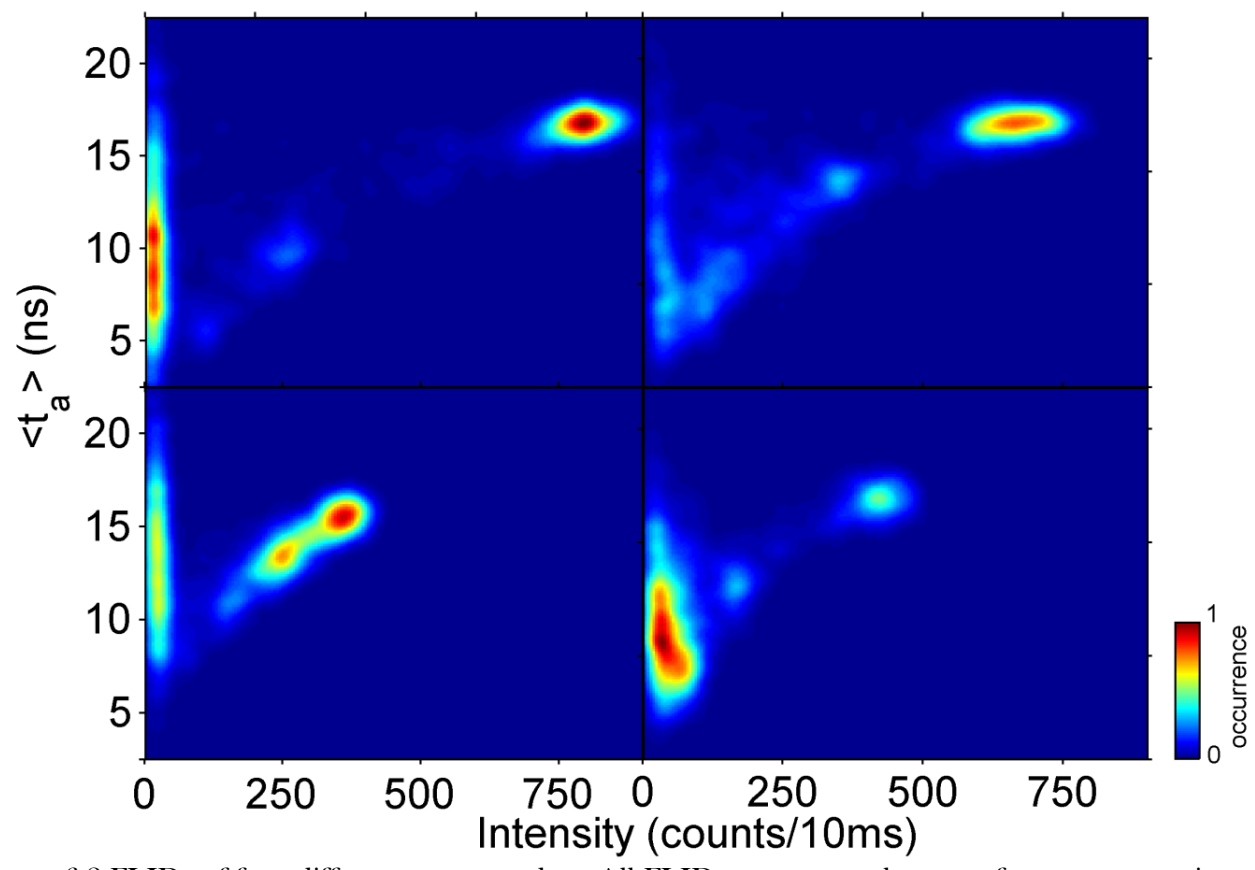

Figure 6.2 FLIDs of four different quantum dots. All FLID axes are set the same for easy comparison. We observe the same monotonically decreasing trend in all FLIDs and see defined peaks that show a different relative spacing depending on the quantum dot. 

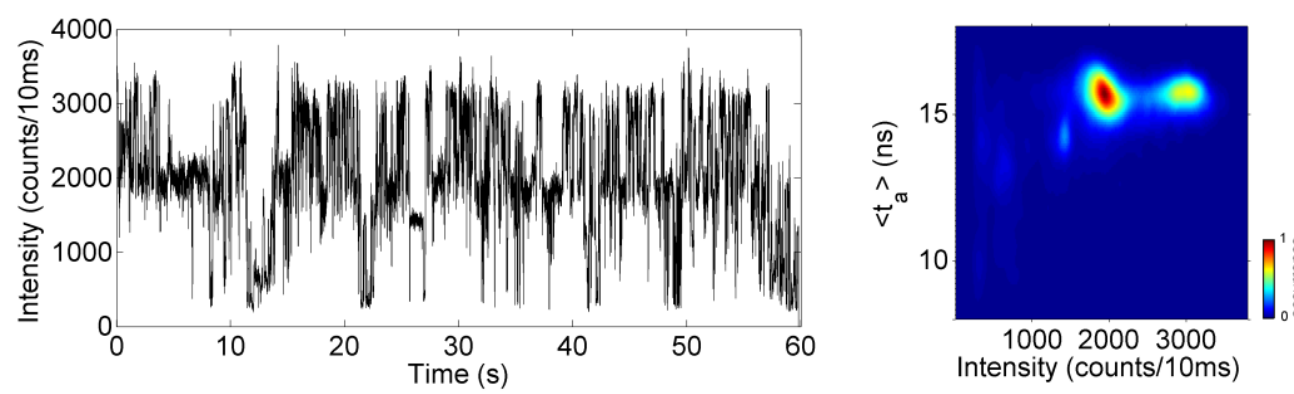

Figure 6.3 (a) Intensity trace of the emission of two quantum dots. (b) The corresponding FLID diagram clearly shows two peaks that have a $\left\langle\boldsymbol{t}_{\boldsymbol{a}}\right\rangle$ around $\sim 15 n s$, which is what we would expect for a bright state of a single quantum dot. Therefore, this FLID shows an emission signature of more than one quantum dot.

the decay characteristics, and since the $\left\langle t_{a}\right\rangle$ of the background signal (the center of the TAC range, 25ns in our case) is at least ten times higher than the $\left\langle t_{a}\right\rangle$ of the distinct peaks. Even a background contribution as small as $10 \%$ pulls the $\left\langle t_{a}\right\rangle$ towards the center of the TAC range. Last, we observe a cloud of data points between the distinct peaks that are not clearly related to the peaks, which we attribute to time-averaged data points.

We found multiple distinct peaks in a majority $(>96 \%)$ of the recorded single quantum dot FLIDs, of which four examples are shown in Figure 6.2. The remaining $4 \%$ of the examined FLIDs were ambiguous or showed signatures of emission from more than one quantum dot.

Again, we notice that the defined peaks occur in all of the FLIDs, but that the peaks are differently spaced and differently populated, depending on the emitter. One explanation for the observed peaks could be that there are more emitters inside the confocal detection volume, each peak representing emission from a single quantum dot. However, this would mean that quantum dots exhibit a wide distribution of lifetimes, which does not comply with the fact that the typical bright state lifetime that is observed in literature is $\sim 20 \mathrm{~ns}[16,18]$.

Moreover, next to the typical examples shown in Figure 6.2, we occasionally observe other emission patterns as shown in Figure 6.3. The intensity trace in Figure 6.3a shows clear signatures of multiple intensity levels, although the intensity of the brightest level is more than two times as intense as the typical intensities observed for the brightest level. The FLID map in Figure 6.3b also shows distinct multiple peaks that can be related to the steady intensity levels observed in the intensity trace, at for example around 1600, 2000, and 3000 counts per 10ms. However, the FLID map (Figure 6.3b) clearly shows two distinct peaks with the average photon arrival time 
a)

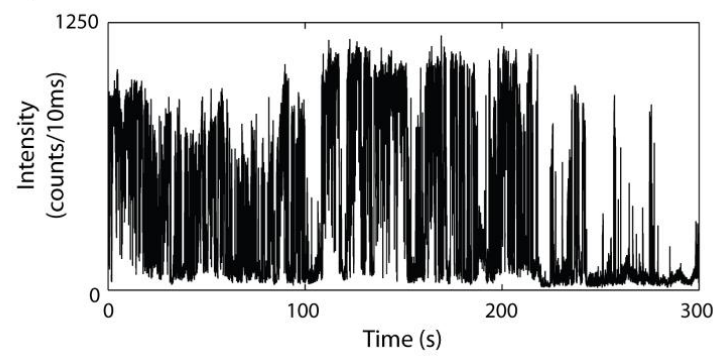

b)

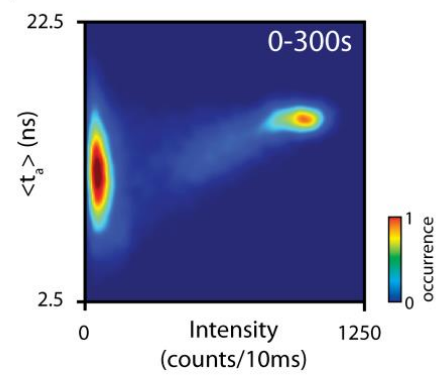

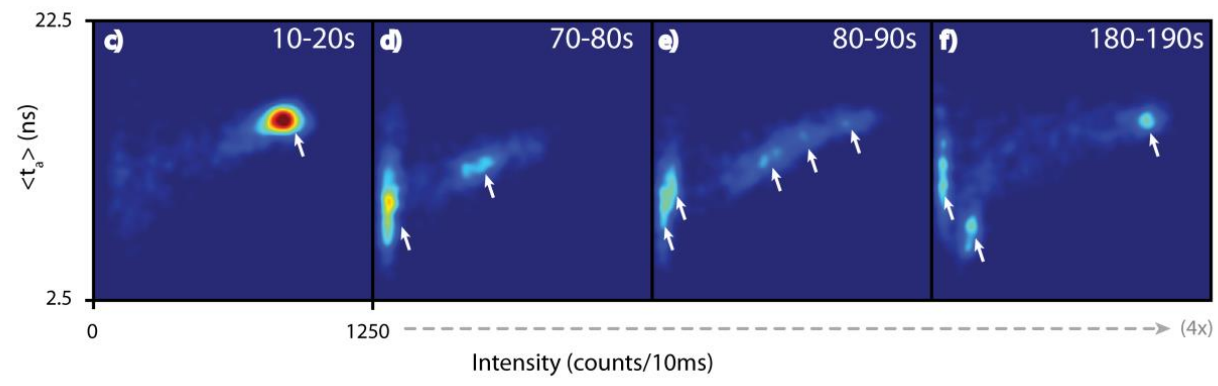

Figure 6.4 A 300s long quantum dot emission trace (a) is analysed using a FLID diagram on the long time scale (b). As reported in literature, we observe a smear of decay-intensity points that suggest a continuum of states. We subdivide the 300s emission trace into smaller traces of $10 \mathrm{~s}$ and compute the FLID of each 10s trace (c-f). We now find distinct peaks in the FLIDs, showing that the definite states are found at the second time-scale.

that we would expect for a bright state of a single quantum dot, meaning that this FLID shows an emission signature of at least more than one quantum dot. The difference between Figure 6.2 and Figure $6.3 \mathrm{~b}$ clearly shows that single quantum dot emission signatures can be readily recognized by qualitative inspection of the FLIDs.

\subsection{Definite states or continuum of states?}

In addition to the 30s long FLIDs, we also recorded single quantum dot emission over a time period of 300s (Figure 6.4a), which is similar to the time frame used in the reports in literature that showed a continuum of intermediate states $(>100 \mathrm{~s})$ [16, 17]. We first calculate the FLID map over the full time frame (Figure 6.4b), and secondly subdivide the total emission trace into 30 shorter traces of 10 s each to follow the evolution of the FLID maps at smaller time scales and compare these short term FLIDs (Figure 6.4c-f) with the long term FLID (Figure 6.4b).

At the long term FLID (Figure 6.4b) we observe a bright peak, a smeared out low intensity peak, and in between we observe a broad smear of the continuum 
of intermediate states, similar to that reported before in literature [16-18, 24]. However, the short term FLIDs (Figure 6.4c-f) clearly show the defined peaks (indicated by the white arrows) as seen before in this chapter. Since the continuum of intermediate states is so clearly visible as a smear in the long term FLID, we can assume that the defined peaks that show a similar trend, from high intensity and slow decay to low intensity and fast decay, are in fact states that each have their defined combination of intensity and decay time. Secondly, we see that the definite states are stable at the second time-scale, but not anymore at the $\sim 100$ s time scale. The fact that the definite states are not long term stable may be the reason why the definite states have not yet been reported in literature, because we used the average photon arrival time instead of lifetime to increase the time-resolution of the emission trace, allowing us to go to shorter time-scales. The main question that still remains is: what is causing the definite states?

\subsection{Nature of the definite states}

In addition to using the FLIDs as a qualitative tool to visualize emitter characteristics, we can also use the FLIDs to investigate the nature of the states that are observed in the FLID image. We collect all photon records of each state in the FLID diagram to study the decay characteristics of the definite states. We record an emission trace of a single quantum dot of which the intensity trace can be seen in Figure 6.5a, which clearly shows blinking between definite states. We calculate the corresponding FLID map (Figure 6.5b) and observe the same definite states, which clearly show a correlated trend between intensity and decay as before. We assign regions-of-interest (ROIs) around each state in the FLID map and use these ROIs to gather all photon records that correspond to each state. For each state, we bundle the microtimes to calculate the decay curves, which are shown for all states in Figure 6.5c.

The brightest state shows mono-exponential decay and we find a growing complexity and fast decay for states with lower intensities, which is in accordance with reports in literature [16-18]. We perform exponential fitting analyses on the decay curves to account for the influence of the uncorrelated background photons and deconvolute the instrument response function from the decay curve. We fit the decay curves with exponential decay functions and add decay components until there is no more significant reduction of the chi-square value. State 1 and 2 were fitted with a mono-exponential, state 3 with a double exponential and state 4 and 5 with a triple exponential decay to provide accurate fitting results (red lines in Figure 6.5c). We calculate the mean lifetime of each state by taking the weighted average of the separate decay components (see section 6.3 for more details) and plot this mean 
a)

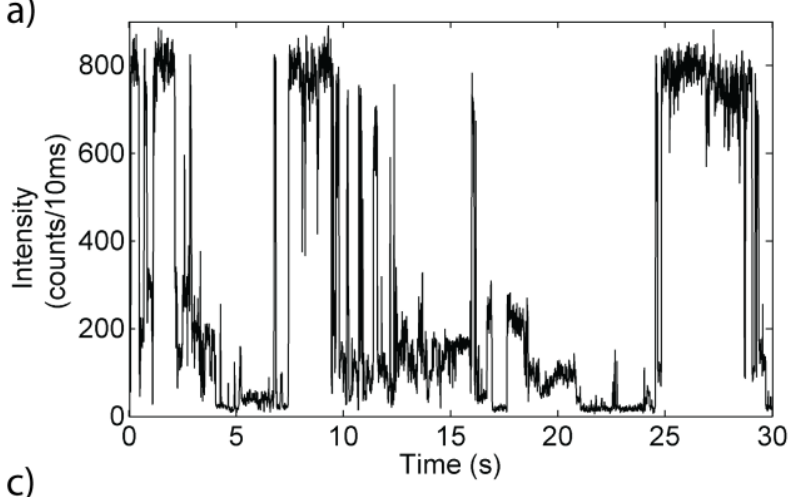

b)

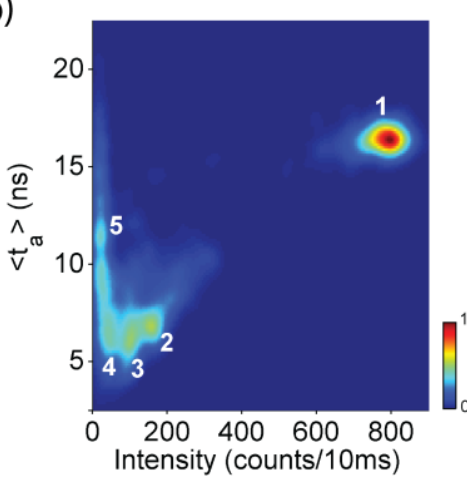

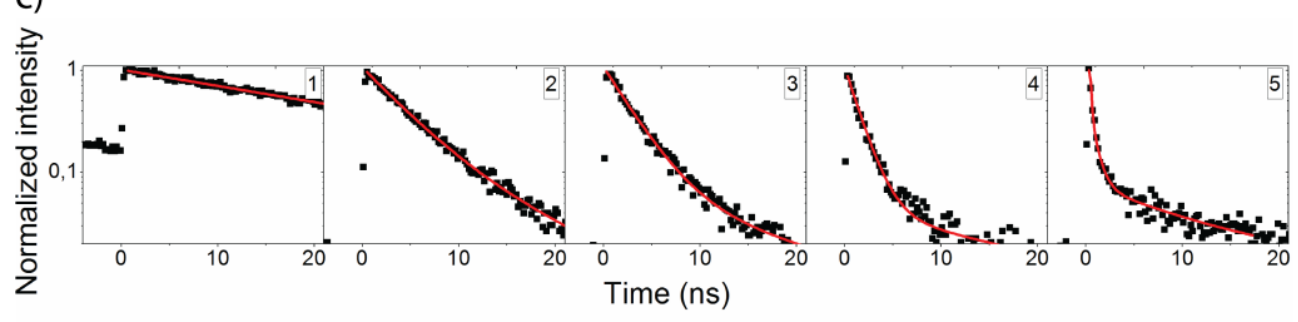

Figure 6.5 Intensity trace of a single quantum dot clearly showing multiple emissive states (a). The corresponding FLID-diagram is plotted (b) and regions-of-interest are selected around the states. The regions-of-interest define which time-points belong to each state and the according photon data is accumulated to gather all the photon arrival time statistics that are known for each state (c). The decay curves are fitted with an exponential model and additional decay components are added until there is no more significant reduction of the chi-square value. We observe a growing complexity and faster decay for states with a lower intensity.

lifetime versus the average intensity of each state (Figure 6.6, black solid square data points).

The mean lifetime shows a linear decreasing trend towards the origin (Figure 6.6). This trend shows that the states occur due to a non-radiative mechanism and that the radiative decay rate remains similar for all states of a quantum dot (a purely radiative process would cause a trend that moves in the cross-diagonal direction since it would be accompanied with faster decay time and higher intensity, which is clearly not the case). Furthermore, the high relative attenuation of the states in the FLID diagrams with respect to the brightest state, up to factors above 20 , shows us that the additional non-radiative decay paths of the intermediate states are strong compared to the decay paths of the brightest state, pointing to a strong non-radiative mechanism that causes the existence of the definite intermediate states. We generally observe similar results for other quantum dots, of which two additional analyses are also shown in Figure 6.6. For all analyses shown in Figure 6.6, we observe the same linear 


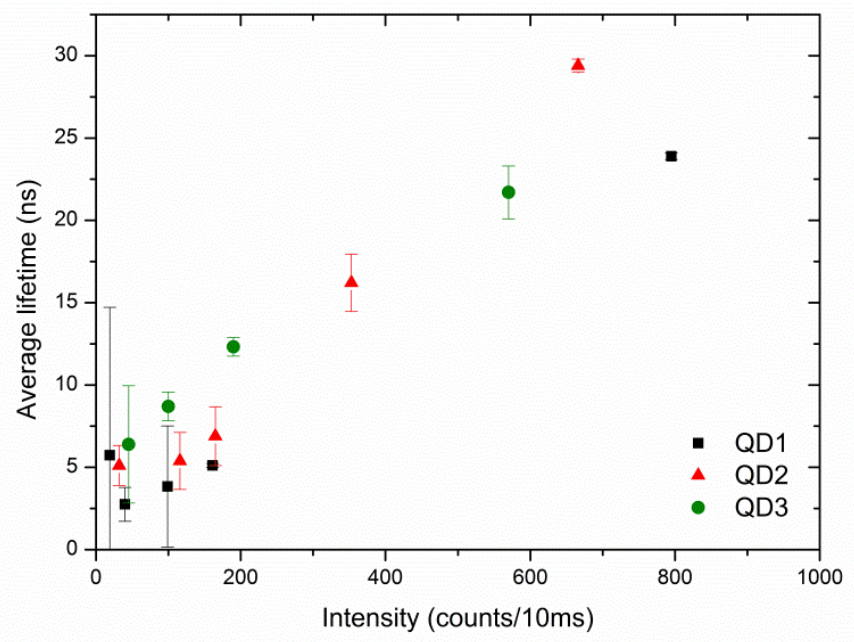

Figure 6.6 Mean lifetime versus intensity for the five states that are selected in Figure 6.5 (black square data points). The mean lifetime shows a linear decreasing trend towards the origin. We generally observe this trend for other quantum dots, of which two more examples are shown by the red triangle and green circle datapoints. These results show that the underlying mechanism for the observed blinking behavior of the definite states is purely non-radiative. The error bars represent the inaccuracy of the average lifetime value and were calculated using the standard deviations of the amplitude and lifetime of the individual exponential components. The errors generally become large for low intensity levels, because the quality of the exponential fit decreases due to the lower number of detected photons.

decreasing trend, supporting that the underlying mechanism for the observed blinking behavior of the definite states is non-radiative.

Comparing our results with literature, we find that Galland et al. [26, 27] also reported distinct peaks in the FLID diagrams of their $\mathrm{CdSe} / \mathrm{CdS}$ core-shell quantum dots. Galland et al. studied blinking of ultra-thick-shell quantum dots and showed that with this type of quantum dots Auger recombination is suppressed due to the thick shell of the quantum dot. They observed blinking between states that show equal emission intensity, but have a radiative decay rate that differ a factor 2 or 3 with respect to the neutral state, which is explained by multifold negative charging of the quantum dot core that creates additional radiative decay pathways. However, in our case intensity attenuation is paired with an increase in decay rate, indicating a quenching process, pointing to an increase in non-radiative pathways instead of radiative pathways. The increase in non-radiative pathways can also not be explained via Auger recombination ( ps decay rate), because Auger recombination would give an increase in non-radiative decay that is so strong that it would result in a single off 
state, and not intermediate states. So what causes the definite states in our FLID diagrams?

\subsection{Defined set of definite states}

In Figure 6.5, we have seen that multiple non-radiative decay paths can co-exist to produce faster and more complex decays for states with lower intensity, but does this mean that a fixed set of states exists for each quantum dot? If there would be a fixed set of states for each quantum dot, the emission trace should show a reoccurrence of the definite states within the same emission trace.

The emission intensity trace in Figure 6.7a clearly shows a reoccurring intermediate intensity level around 1000 counts per $10 \mathrm{~ms}$. The corresponding FLID map clearly shows the same definite state as observed in the emission intensity trace (Figure 6.7b, indicated by the arrow). The narrow width of the peak in the FLID map indicates a high specificity for the exact same combination of intensity and $\left\langle t_{a}\right\rangle$. The height of the peak in the FLID map shows that the specific combination of intensity and $\left\langle t_{a}\right\rangle$ is frequently present in the emission trace, meaning that the quantum dot remained in that state for a long time, or that the state is revisited multiple times within the same emission trace. The intensity trace shows that the definite state is revisited multiple times within the same emission trace.

To support the reoccurrence of the definite states, we analyze the reoccurrence of multiple definite states of the single quantum dot emission trace shown in Figure 6.8ab at a short period in time (8-15s), to zoom-in on a specific part of the trace that shows reoccurrence of multiple definite states. We observe correlated intensity and $\left\langle t_{a}\right\rangle$ corresponding to the definite states, which are also

a)

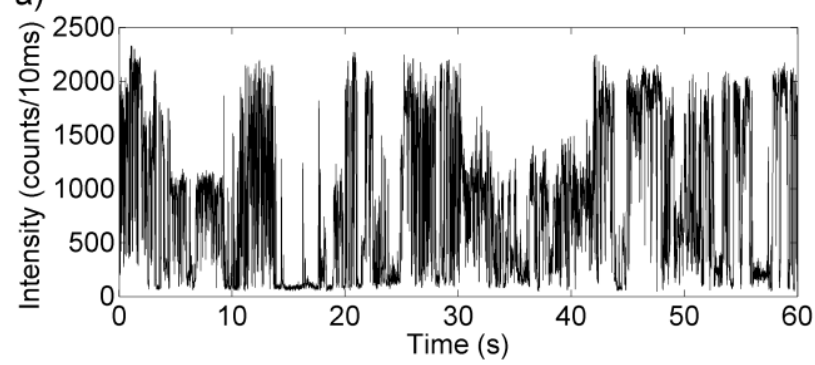

b)

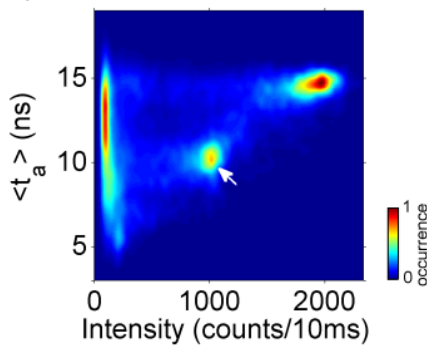

Figure 6.7 (a) Emission intensity trace of a single quantum dot. We observe reoccurring intensity levels during the same emission intensity trace. (b) The corresponding FLID clearly shows the same definite states that are found as reoccurring intensity levels in the intensity trace (indicated by the arrow). The narrow width of the definite states in the FLID indicates that the exact same combination of intensity and $\left\langle\boldsymbol{t}_{\boldsymbol{a}}\right\rangle$ is revisited multiple times within the same emission trace. 

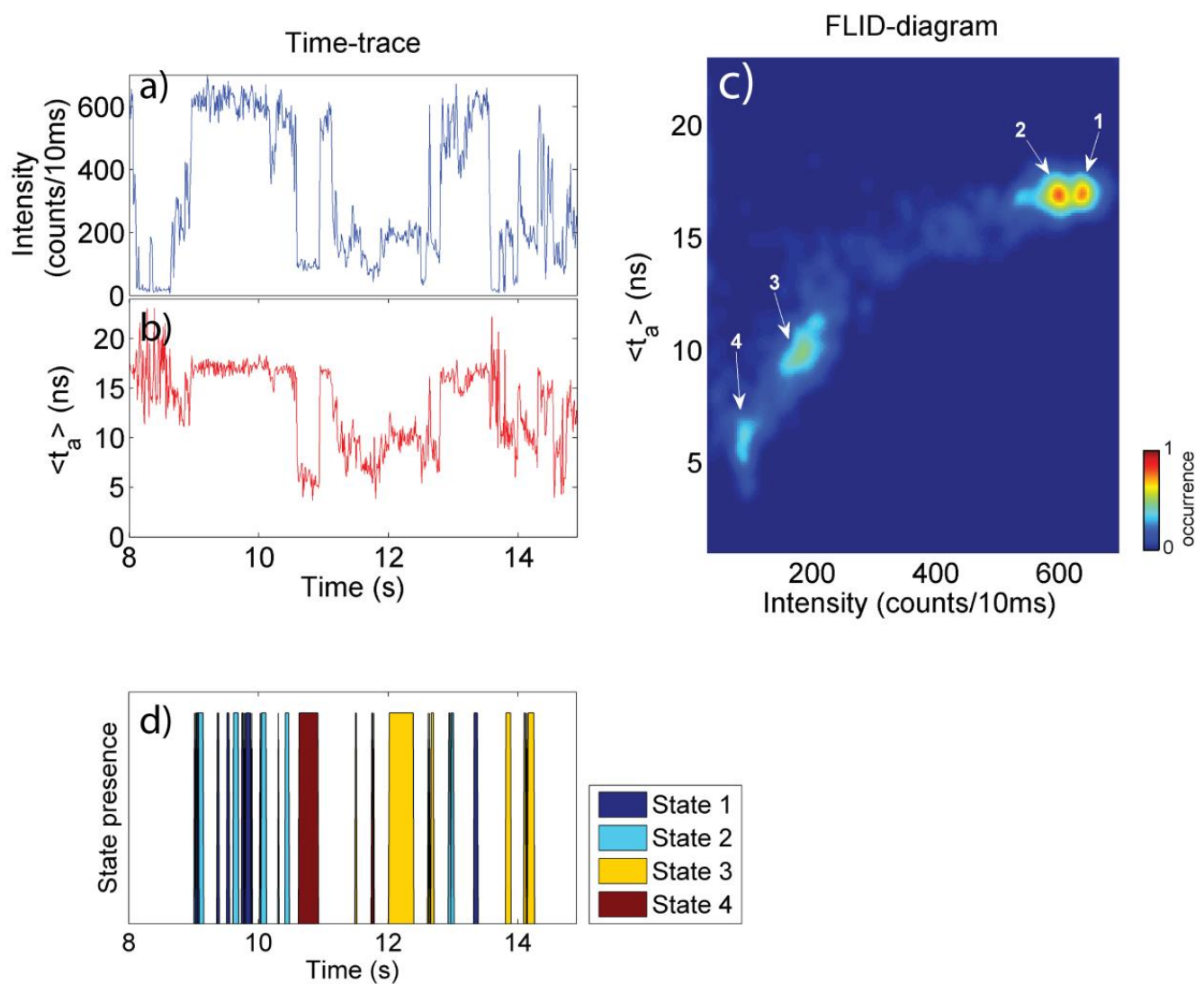

Figure 6.8 Example of a quantum dot emission trace (ab). We define ROIs around the states that we observe in the FLID diagram (c) and categorize time-bins based on the selected ROIs. These time-bins are then translated to binary masks that indicate the presence of the states over a $5 \mathrm{~s}$ period of time (d). We also exclude state occurrences where the period is shorter than $50 \mathrm{~ms}$, to exlude false positives, which results in the empty gaps between the binary masks. We observe a variety of state durations, which is expected from the power law distributed blinking behavior of these quantum dots. Interestingly, we observe reoccurence of the states within the same emission trace, showing that these states represent preferred states that the quantum dot revisits after some period of time.

clearly visible in the FLID map (Figure $6.8 \mathrm{c}$, indicated by the numbered states). Using a similar approach as in Figure 6.5, we define ROIs around the states and categorize the photon record into states, but this time we use the categorization to mark the corresponding time-bins in the emission trace to study the reoccurrence of the definite states within the same emission trace. We select the time-bins that correspond to these states and create binary masks for each of these states. Furthermore, these binary masks are filtered to select only those state occurrences that lasted longer than $50 \mathrm{~ms}$, to exclude possible false positives that could occur due to noise, time-averaged emission levels or fast blinking events. The resulting binary 
masks are shown in Figure 6.8d. The white gaps in between the binary masks of the states are the regions where the false positives were discarded. Interestingly, we observe reoccurence of the states within the same emission trace, showing that these states represent preferred combinations of intensity and decay time that the quantum dot revisits after some period of time.

\subsection{Modified trapping model}

To summarize, we report on the existence of specific sets of intermediate states that each have a defined combination of intensity and decay, where the set of intermediate states varies from emitter to emitter [17]. The definite states show similar behavior as the continuous distribution of intermediate states, showing a strong linear correlation between fluorescence intensity and decay, and show increasingly complex decay characteristics for states with lower intensity, indicating that fluctuations in non-radiative decay are dominant for the intermediate states, equivalent to what has been reported in literature [16-18] for the continuum of emission intensities. Interestingly, we observe that the definite states are revisited over time at the seconds time-scale, but become unstable at the minute time-scale, which is observed as a continuous distribution of states in the FLID diagram, thereby showing that the continuous distribution of the intermediate states is in fact a collection of all definite states that are stable at the second time-scale. The narrow peaks in the FLID diagram at the seconds time-scale not only illustrate that the combination of intensity and decay for the definite states is highly specific, but also shows that the specific combination of intensity and decay frequently re-occurs and shows that even at the millisecond time-scale each definite state is a multiplexed state, meaning that each state has a fixed set of substates that are always populated in the same manner, each time giving the same complex decay characteristics.

The fluctuating trap model suggests that the distance from the trap state to the core determines the non-radiative decay time, giving faster decay for traps that are closer to the core, and vice versa. The wavefunction of holes reaches further away from the core, and into the shell, compared to the wavefunction of the electrons that is mostly confined to the core [28], suggesting that holes are more likely to be trapped outside the core and cause the quenching process. Furthermore, the wavefunction of the holes shows the maximum probability around the core-shell interface and declining probability deeper into the shell. This means that hole trapping is more likely to occur close to the core-shell interface and less likely to get trapped deeper into the shell region. Following the same line of argumentation, we 
explain the multiplicity of the definite states by a trapped charge that is confined to a small domain outside the core region (see Figure 6.9).

Within the confined space of a domain, the trapped charge is allowed to jump to a discrete set of acceptor sites. The domains represent the definite states and the acceptor sites represent the substates. The distance between a domain and the core determines the overall decay rate of a definite state, as with the conventional fluctuating trap model. Within a domain, the trapped charge hops from one acceptor site (substate) to the other at a time-scale far below the $10 \mathrm{~ms}$ binning time, giving a time-averaged complex decay characteristic, where the complexity of the decay characteristic depends on the number of acceptor sites. We observe an increasing decay complexity for definite state with lower intensity, meaning that the number of acceptor sites increases when the domain is closer to the core region.

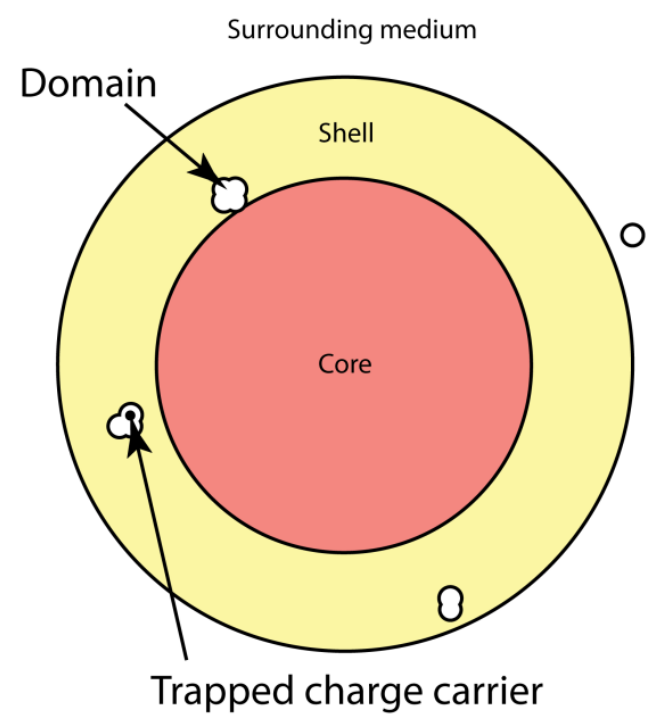

Figure 6.9 Proposed trapping model for the existence of the definite multiplexed intermediate states. A trapped charge is confined to a small domain outside the core region, which can be either close to the core/shell interface, inside the shell or in the surrounding medium. Within the confined space of a domain, the trapped charge is allowed to jump to a discrete set of acceptor sites. The domains represent the definite states and the acceptor sites represent the substates. The distance between a domain and the core determines the overall decay rate of a definite state, as with the conventional fluctuating trap model. Within a domain, the trapped charge hops from one acceptor site (substate) to the other at a time-scale far below the $10 \mathrm{~ms}$ binning time, giving a timeaveraged complex decay characteristic, where the complexity of the decay characteristic depends on the number of acceptor sites. Since we observe faster and more complex decay characteristics for states with lower intensity, domains have an increased number of acceptor sites when being closer to the core. 
This increase in number of acceptor sites is expected from the charge carrier wavefunctions for $\mathrm{CdSe} / \mathrm{ZnS}$ heterostructures [28], which predict the highest density of trap states to be near the $\mathrm{CdSe} / \mathrm{ZnS}$ interface and in the $\mathrm{ZnS}$ shell owing to alloying dislocations. The population of the acceptor sites by the trapped charge is related to the relative accessibility of the acceptor sites within a domain, and determines the relative contributions of substates to the decay characteristic of a definite state. The relative accessibility of the acceptor sites within a domain is stable at the seconds time-scale, but alters most likely due to thermal fluctuations at the minute time-scale, giving a broad distribution of intermediate states rather than definite intermediate states. Meanwhile, the trapped charge quenches the emissive state, until it recombines and neutralizes the quantum dot.

\subsection{Conclusion}

To conclude, we studied in detail the emission of single $\mathrm{CdSe} / \mathrm{ZnS}$ core-shell quantum dots using FLID diagrams and found defined sets of states that differ per quantum dot, where each definite state has its own characteristic combination of intensity and decay time. This finding extends our understanding of quantum dot blinking, since up till now only a continuum of states has been observed for $\mathrm{CdSe} / \mathrm{ZnS}$ core-shell type quantum dots. We observed faster decay rates and more complex decay characteristics for definite states that show a decreased intensity, in agreement in literature for the continuum of states [16-18, 24]. The linear correlation between intensity and decay time shows that the underlying mechanism is, for the definite states as well as the continuum of states, driven by non-radiative processes. In addition, we observed that these definite states reoccur within the same emission trace, indicating preferred states that are revisited over time. The presence of the defined set of states, and the increasing complexity of decay characteristics for states with lower intensity, points towards having multiplexed states, where each state can have a number of substates that in turn each have their own decay paths. A detailed comparison between long term and short term FLID diagrams showed that the multiplexed structure of the definite states can alter over long periods of time and explains why a continuum of states was found in literature. We propose a model explaining our observations, in which each domain (state) can have multiple acceptor sites (substates). The distance from a domain to the core determines the overall decay time, the number of acceptor sites determines the complexity of the decay characteristics, and the relative accessibility of the acceptor sites within a domain determines the population of the substates. At the minutes time-scale the accessibility of the acceptor sites alters, producing a broad distribution of intermediate states 
rather than a defined set of intermediate states. This model explains our observations and is consistent with findings in other reports in literature.

\section{REFERENCES}

1. Kuno, M., et al., Nonexponential "blinking" kinetics of single cdse quantum dots: A universal power law behavior. Journal of Chemical Physics, 2000. 112(7): p. 31173120.

2. Shimizu, K.T., et al., Blinking statistics in single semiconductor nanocrystal quantum dots. Physical Review B, 2001. 63(20).

3. Frantsuzov, P.A. and R.A. Marcus, Explanation of quantum dot blinking without the long-lived trap hypothesis. Physical Review B, 2005. 72(15).

4. $\quad$ Frantsuzov, P., et al., Universal emission intermittency in quantum dots, nanorods and nanowires. Nature Physics, 2008. 4(7): p. 519-522.

5. Hoogenboom, J.P., et al., Memory in single emitter fluorescence blinking reveals the dynamic character of nanoscale charge tunneling: Journal of Physical Chemistry C, 2008. 112(9): p. 3417-3422.

6. $\quad$ Spinicelli, P., et al., Bright and grey states in cdse-cds nanocrystals exhibiting strongly reduced blinking. Physical Review Letters, 2009. 102(13).

7. Jones, M., S.S. Lo, and G.D. Scholes, Quantitative modeling of the role of surface traps in cdse/cds/zns nanocrystal photoluminescence decay dynamics. Proceedings of the National Academy of Sciences, 2009. 106(9): p. 3011-3016.

8. Cragg, G.E. and A.L. Efros, Suppression of auger processes in confined structures. Nano Letters, 2010. 10(1): p. 313-317.

9. Zhao, J., et al., Challenge to the charging model of semiconductor-nanocrystal fluorescence intermittency from off-state quantum yields and multiexciton blinking. Physical Review Letters, 2010. 104(15).

10. Early, K.T., et al., Polarization-driven stark shifts in quantum dot luminescence from single cdse/oligo-ppv nanoparticles. Nano Letters, 2010. 10(5): p. 1754-1758.

11. Ye, M. and P.C. Searson, Blinking in quantum dots: The origin of the grey state and power law statistics. Physical Review B, 2011. 84(12): p. 125317.

12. Bharadwaj, P. and L. Novotny, Robustness of quantum dot power-law blinking. Nano Letters, 2011. 11(5): p. 2137-2141.

13. Klimov, V.I., et al., Quantization of multiparticle auger rates in semiconductor quantum dots. Science, 2000. 287(5455): p. 1011-1013.

14. Efros, A.L. and M. Rosen, Random telegraph signal in the photoluminescence intensity of a single quantum dot. Physical Review Letters, 1997. 78(6): p. 11101113.

15. Verberk, R., A.M. van Oijen, and M. Orrit, Simple model for the power-law blinking of single semiconductor nanocrystals. Physical Review B, 2002. 66(23).

16. Schlegel, G., et al., Fluorescence decay time of single semiconductor nanocrystals. Phys Rev Lett, 2002. 88(13): p. 137401.

17. Zhang, K., et al., Continuous distribution of emission states from single cdse/zns quantum dots. Nano Letters, 2006. 6(4): p. 843-847. 
18. Fisher, B.R., et al., Emission intensity dependence and single-exponential behavior in single colloidal quantum dot fluorescence lifetimes. Journal of Physical Chemistry B, 2004. 108(1): p. 143-148.

19. Bawendi, M.G., et al., Luminescence properties of cdse quantum crystallites: Resonance between interior and surface localized states. The Journal of Chemical Physics, 1992. 96(2): p. 946-954.

20. Issac, A., et al., Influence of the dielectric environment on the photoluminescence intermittency of cdse quantum dots. Chemphyschem, 2012. 13(13): p. 3223-3230.

21. Califano, M. and F.M. Gómez-Campos, Universal trapping mechanism in semiconductor nanocrystals. Nano Letters, 2013. 13(5): p. 2047-2052.

22. Lakowicz, J.R., Principles of fluorescence spectroscopy. 3rd ed. 2006.

23. $\quad$ Prummer, M., et al., Single-molecule identification by spectrally and time resolved fluorescence detection. Analytical Chemistry, 2000. 72(3): p. 443-447.

24. Schmidt, R., C. Krasselt, and C. von Borczyskowski, Change point analysis of matrix dependent photoluminescence intermittency of single cdse/zns quantum dots with intermediate intensity levels. Chemical Physics, 2012(0).

25. Amecke, N. and F. Cichos, Intermediate intensity levels during the emission intermittency of single cdse/zns quantum dots. Journal of Luminescence, 2011. 131(3): p. 375-378.

26. Galland, C., et al., Lifetime blinking in nonblinking nanocrystal quantum dots. Nature communications, 2012. 3: p. 908.

27. Galland, C., et al., Two types of luminescence blinking revealed by spectroelectrochemistry of single quantum dots. Nature, 2011. 479(7372): p. 203207.

28. Early, K.T. and D.J. Nesbitt, Size-dependent photoionization in single cdse/zns nanocrystals. Nano Letters, 2013. 13(10): p. 4844-4849. 



\section{Chapter 7}

\section{Future applications of multimodal FLIM}

The preceding chapters used the multimodal platform mainly for the studying photophysics of single emitters. However, the high sensitivity of the platform is also advantageous for ensemble studies, allowing for fast scanning while at the same time having the possibility to analyze specific domains at the sample. Although multimodal FLIM is already being used in a wide range of applications (as discussed in chapter 2), we also worked on the development of new methods and applications for multimodal FLIM. In this chapter, we will describe some of these applications, giving examples of both a method and an application that required the multimodality of the platform to support their further development.

A part of this chapter has been published in Journal of Materials Chemistry, 2012, 22(32), pages 16606-16610, Patterning perylenes on surfaces using thiol-ene chemistry, doi: 10.1039/C2JM32610H 


\subsection{Measurement of the quantum efficiency of fluorophores}

\subsubsection{Conventional method}

In chapter 1 we discussed the quantum efficiency $(\mathrm{QE})$ of fluorophores, which is a parameter that shows how efficiently a fluorophore converts absorbed photons into emitted photons. A QE can be lower than unity if during the relaxation from the excited state to the ground state energy is lost due to non-radiative processes, e.g. heat, rather than fluorescent radiation. A widely used method to measure the QE of a fluorophore is to compare the emission intensity of the fluorophore of interest with that of a reference fluorophore having the same absorbance and the same excitation wavelength [1], and is currently still considered as the standard method to determine the QE [2].

However, as already pointed out in chapter 1, this standard method always underestimates the true $\mathrm{QE}$ because emitters can also (temporarily) reside in a dark state while still absorbing, which lowers the net fluorescence emission intensity of the fluorophore of interest and in turn causes an underestimation of the true QE. In fact, the measured $\mathrm{QE}$ of a fluorophore of interest can even be overestimated in case that the measured QE of the reference fluorophore is underestimated as well.

\subsubsection{Fluorescence lifetime based method}

Our group recently developed an intensity independent method to accurately determine the $\mathrm{QE}$ of a fluorophore [3]. In this method, the local optical environment of the emitter is altered to induce modifications in fluorescence decay rate [4], the inverse of lifetime. These decay rate modifications are well described in literature [4-11], especially for our case where an isotropically emitting thin film of dipoles is being positioned at defined distances from a plane mirror. The decay rate modifications can be derived from Fermi's golden rule [12], and predicts that the radiative decay rate is proportional to the projected local density of states (LDOS), and explicitly depends on the distance to the mirror $d$ and the emission transition frequency $\omega$ :

$$
\gamma(\omega, d)=\frac{\operatorname{LDOS}(\omega, d)}{\operatorname{LDOS}_{0}(\omega, d)} \cdot \gamma_{r a d, 0}+\gamma_{n r a d, 0}
$$

where the subscript zero denotes the nominal values of the parameters, in absence of the mirror and in a homogeneous environment. The total decay rate shows a damped oscillation as a function of distance to the mirror, which is for example 
a)

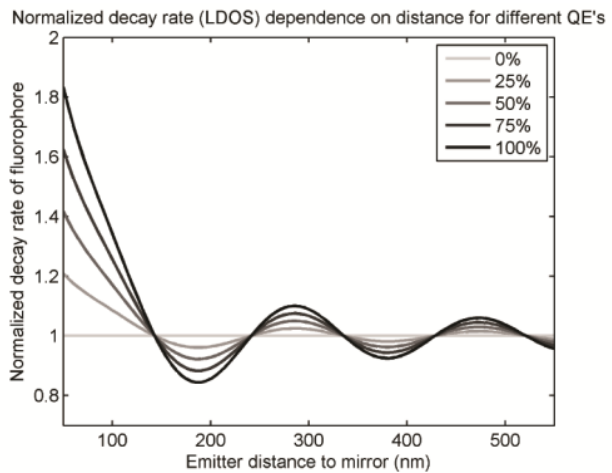

b)

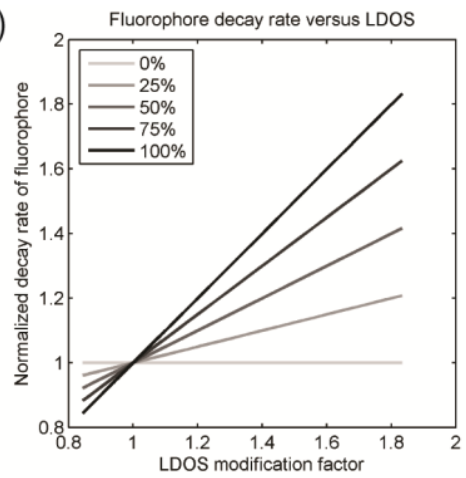

Figure 7.1 (a) LDOS curves for 5 different quantum efficiencies, calculated for a fluorophore that is at a distance from a silver mirror, 1.5 refractive index of the medium and $550 \mathrm{~nm}$ transition wavelength. (b) LDOS curves of a fluorophore of interest plotted for 5 different QE's versus the LDOS.

shown in Figure 7.1a, where the decay rate is normalized by the decay rate in absence of the mirror $\left(\gamma / \gamma_{0}\right)$. From a classical point-of-view, this oscillation can be seen as the reflected emission light interfering with the emitter, either driving the emitter harder (in-phase, increased LDOS) or slowing it down (out-of-phase, reduced LDOS) [6].

As can be seen in Figure 7.1a, the rate modification is strongest for fluorophores with a high QE, having a high relative radiative rate compared to the non-radiative rate. For fluorophores with a low QE, the total decay rate remains essentially unaffected. By plotting the measured total decay rate versus the LDOS modification factor (simulated in Figure 7.1b), which is calculated from the theoretical model, both the radiative and the non-radiative decay rate can be derived from the slope and offset, respectively. These two rates represent the typical fluorophore decay rates that occur in a homogeneous environment and in absence of the mirror, and allow one to determine the $\mathrm{QE}$ of the fluorophore of interest.

The LDOS manipulation method only requires a precise control of the separation distance between the emitter and mirror, because the LDOS explicitly depends on the distance between the emitter and the plane mirror, which in practice can be controlled in many ways. Our group controlled this distance by evaporating a flat spacer layer of $\mathrm{SiO}_{2}$ with a defined thickness onto a flat mirror, and spin-coating a thin film of fluorophores on top of the spacer layer. The lifetime is measured across a wide scanning area to increase the statistical significance of the measurement, and to determine the total decay rate accurately. The decay rate measurement is repeated for a set of samples with varying emitter-mirror distances. By plotting the total decay 
rate $(\gamma)$, that is, the inverse of the measured lifetime, versus the LDOS modification, one can determine the radiative rate and non-radiative rate, as explained above.

\subsubsection{Introducing a curved reflective mirror}

The method described above determines the unbiased QE, but does require a large set of samples, because each sample has a fixed emitter to mirror distance. We started a pilot study to improve this method by applying a distance gradient in one sample [13, 14], allowing one to manipulate the LDOS within one sample rather than a set of samples, which is more accurate, reduces the random errors and has only one systematic error, and is also less time-consuming. As explained in the previous section, the two general requirements are the precise control over the emitter-mirror distance, and accurate measurements of the fluorescence lifetime. Furthermore, the sample needs to be scanned laterally with high precision, to perform the LDOS modification experiment within a single sample. Our multimodal platform meets all requirements, accessing the emitter-mirror distance using the spectrograph, measuring lifetime using TCSPC, and precise lateral scanning with the piezo-stage, as will be explained in detail below.

The distance gradient is introduced by using a curved mirror rather than the plane mirror (see Figure 7.2a). The curved mirror is made by sputtering the convex surface of an off-the-shelf plano-convex lens (Thorlabs LA1433, $\mathrm{f}=150 \mathrm{~mm} \pm 1 \%$, $\mathrm{ROC}=77.3 \mathrm{~mm}$ ) with a $3 \mathrm{~nm}$ chromium layer, and a $200 \mathrm{~nm}$ silver layer on top. The sag of the convex surface introduces the distance gradient, and thus an LDOS gradient across the sample later on. First, we characterize the sag of the convex surface by placing the coated convex surface onto a semi-reflective silver coated

a)

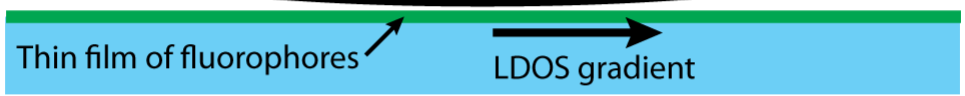

b)

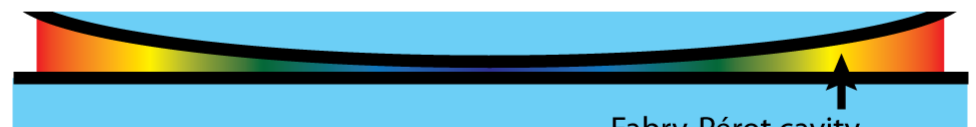

Fabry-Pérot cavity

Figure 7.2 (a) Introducing an LDOS gradient using a curved mirror. (b) Fabry-Pérot cavity based on the same curved mirror as used in (a). 
a)

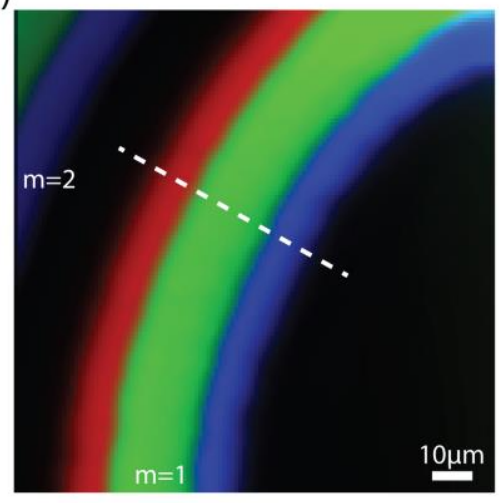

b)

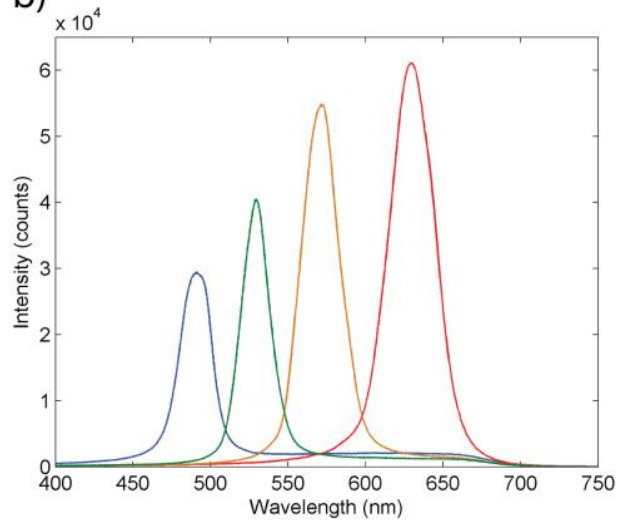

Figure 7.3 (a) 'Rainbow' rings appear due to the sag dependent resonance wavelength in the FabryPérot cavity. The RGB map was reconstructed from the resonance peaks in the emission spectra (b). The resonance peak wavelengths are used to calculate the sag curve of the convex surface, which in turn provides a reference for the LDOS gradient.

microscope coverglass, creating a Fabry-Perot cavity (Figure 7.2b). We illuminate the cavity with white light from a halogen lamp to be able to observe a variety of wavelength resonances within the cavity, which is observed as 'rainbow' rings as shown in Figure 7.3a. We used the emission spectrometer from our multimodal platform to measure the transmission spectrum across the cavity, and accurately determine the local resonance wavelengths (Figure 7.3b). The sag of the reflective convex surface is calculated using the Fabry-Pérot equation for cavity resonances (equation 7.2).

$$
d=\frac{n \lambda}{2} m
$$

where $d$ is the sag, $n$ the refractive index of the medium within the resonator (in our case air), $\lambda$ the resonance wavelength, and $m$ the order of the resonance being a positive integer. The sag curve is fitted with a second order polynomial, which is used later on as a reference for the LDOS gradient.

The sputtered lens is then put onto a thin film of emitters, and touching at the vertex of the lens. The thin film of emitters has been made by spin coating $1 \mu \mathrm{M}$ Rhodamine6G (fluorophore with a high QE) in 2\%WT PVA at 6000RPM for 30 seconds on a \#1.5 microscope coverglass. The medium in-between the curved mirror and thin film roughly matches the refractive index of the cover glass. We used a $5 \mathrm{\mu l}$ drop of Norland Optical Adhesive (NOA61) as a medium between the layer 
a)

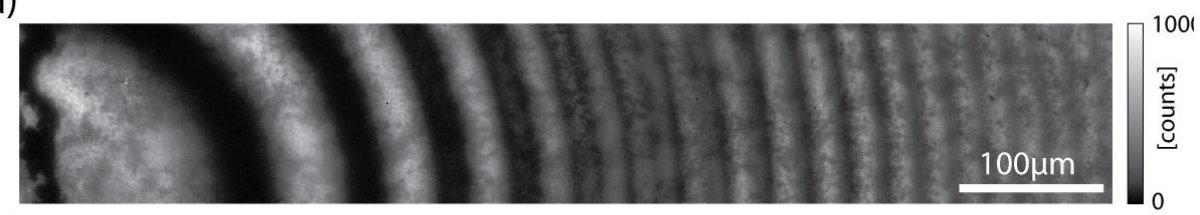

b)

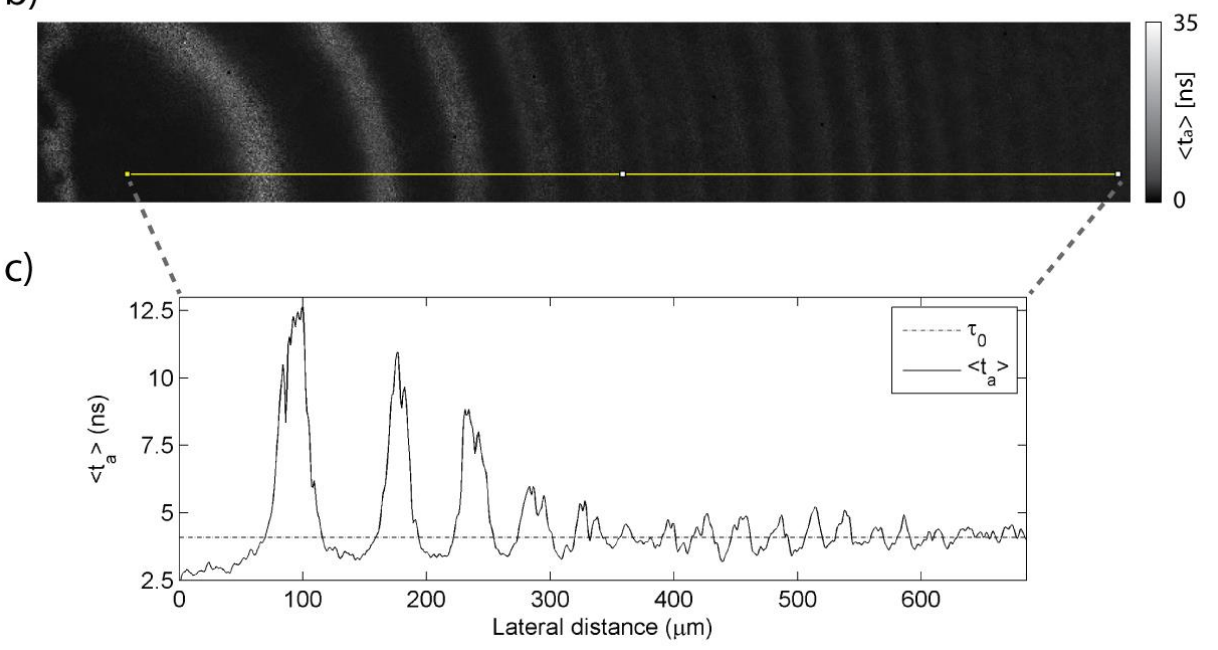

Figure 7.4 (a) Stitched fluorescence intensity map of repeated area-scans around the vertex of the curved mirror, and the corresponding average photon arrival time $\left\langle\boldsymbol{t}_{\boldsymbol{a}}\right\rangle$ map (b). The lifetime oscillation is clearly visible in the line-profile (c), where the dashed line indicates the literature value of the lifetime for Rhodamine $6 \mathrm{G}$ in absence of the mirror.

of emitters and the curved mirror. We wait for 15 minutes until the curved mirror has sunk to its lowest point due to gravity. We illuminated our sample, in the configuration as shown in Figure 7.2a, with an excitation wavelength of $510 \mathrm{~nm}$ and raster scanned the sample around the vertex of the curved mirror and observe intensity (Figure 7.4a) and lifetime oscillations (Figure 7.4b). Due to our limited scanning range $\left(150 \mathrm{x} 150 \mu \mathrm{m}^{2}\right)$, we repeatedly translated the sample about $100 \mu \mathrm{m}$ to reach a larger area, and afterwards stitched all recorded area-scans with a sub-pixel accurate stitching algorithm (ImageJ toolbox Fiji, [15]). The intensity rings show a high contrast ranging from 1000 to 10 counts, representing modulation of the fluorophore emission across the sample. Ironically, the high contrast in intensity complicates the lifetime analyses, since some points have such low intensity (1050 counts), that these point yield inaccurate exponential fit curves and result in unreliable estimation of the fluorescence lifetime.

In chapters 5 and 6 , we showed that the average photon arrival time is a way to estimate the mean lifetime, especially in case of low photon counts. In addition, 108 
the mean lifetime is equal to the fluorescence lifetime in case of mono-exponential decay. Since we only expect to observe mono-exponential decay characteristics in this sample, we can thus estimate the lifetime by considering the average photon arrival time. The average photon arrival time is corrected for the onset of the instrument-response-function (IRF) of the source and corrected for the convolution with the IRF. The resulting $<t_{a}>$-map is shown in Figure 7.4b.

In the line-profile of the $\left\langle t_{a}>\right.$-map (Figure 7.4c, smoothed with a Gaussian filter, $\sigma=1 \mu \mathrm{m}$ ), we clearly observe a damped oscillation, which we would expect to occur due to the LDOS manipulation across the sample. The dashed line indicates the literature lifetime value for Rhodamine $6 \mathrm{G}$, and shows that the local optical environment does not affect the emission of the fluorophores anymore far away from the curved mirror's vertex, in accordance with our expectation. In addition, the oscillations become stronger when the fluorophore is closer to the vertex, and thus closer to the mirror surface. However, the magnitude of the peaks in the $\left\langle t_{a}>\right.$ profile is higher than expected from the LDOS model. We noticed that the sample points that produce these high magnitude peaks also show a low photon count. In the previous chapter, we already recognized a strong weight for the $\left\langle t_{a}\right\rangle$ towards the middle of the TAC range (25ns) for intensities that are close to the background level. Therefore, we suspect that the background signal is too high and does not give an accurate estimate of the lifetime for low intensities, and is thus causing the strong dips in the profile of the normalized decay rate. For future experiments, we highly recommend to collect more photons to have a better estimate of the lifetimes, and to be free from artefact due to a low number of photons. Furthermore, we experienced difficulties in having a point of hard contact between the curved mirror and the fluorophore layer, for reasons that we do not fully understand yet. This uncertainty in the offset of the distance gradient makes is hard to relate the sample coordinates back to the induced LDOS, and thus does not allow us to go to the final step, where we would have plotted the measured decay rate versus the LDOS modification (obtained from the distance gradient) to obtain the QE of the fluorophore.

The method to obtain the QE in a single sample using a LDOS gradient appears to be promising. Recently, others already developed similar platforms that can be used to measure the QE of a mixture of fluorophores [13] or even use an identical platform [14], having both the LDOS gradient and Fabry-Pérot cavity simultaneously, showing the potential of this method, and allow for the precise determination of the quantum efficiency. 


\subsection{Surface patterning of fluorophores}

The multimodal platform also proved to be a crucial part in surface patterning studies, because both imaging and characterisation were simultaneously required to confirm correct deposition of the fluorophores onto inorganic substrates. In general, the multimodal platform can be used for any surface patterning using fluorophores, of which we describe two examples in this section. In one example a substrate is patterned chemically, and the other example uses a nano-dispensing technique to physically deposit fluorophore patterns.

As already mentioned in the introduction, one of the reasons for the broad applicability of perylene dyes is their favorable chemical properties, allowing for easy functionalization, which for example opens up possibilities for controlled binding of organic molecules and compounds to silicon chips. The Molecular Nanofabrication (MNF) Group at the University of Twente developed different methods to pattern thiolated silicon surfaces with alkene-terminated perylene dyes [16], making use of a so-called click reaction, which is a novel reaction method that has proven to be an elegant and successful route to anchor (bio)molecules to surface. In our collaboration with the MNF group, we used our multimodal platform to support their research by both imaging and characterizing their perylene patterned surfaces [16].

The recorded intensity images (see Figure 7.5a) confirmed the highresolution deposition of structured perylene monolayer line-patterns with sizes in the micrometer range. In addition, we characterized the fluorescence of the line-patterns both spectrally and in a time-resolved manner. The emission spectrum of the deposited lines showed a broad aggregation band with a peak at $659 \mathrm{~nm}$, which

a)

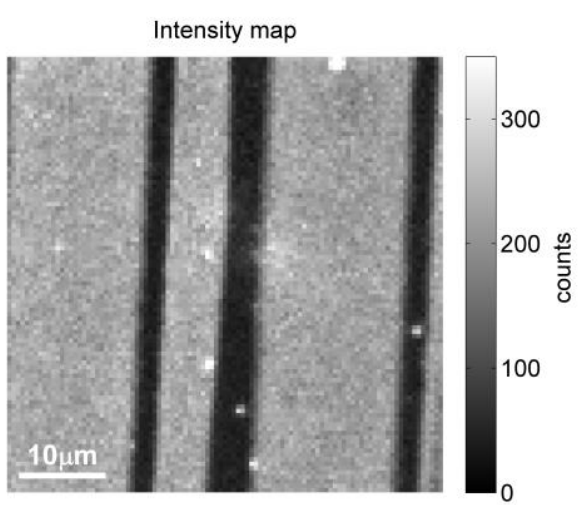

b)

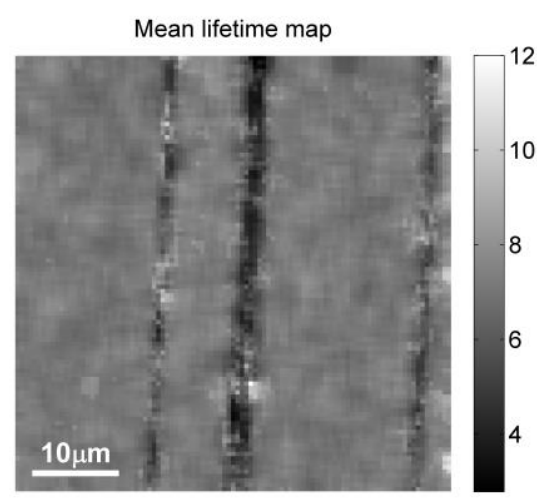

Figure 7.5 Nanolithography (NIL) patterned surface. (a) The fluorescence intensity map shows the perylene lines. (b) The lifetime map indicates that the lines show a long lifetime compared the regions in-between the lines, which indicates aggregates at the location of the lines, as expected. 
confirmed the deposition of a thin film of charge-transfer and excimer species of vacuum deposited perylene. Moreover, the spectrum also indicated a small fraction of monomeric or defect species, showing up as a low-intensity band with a peak at $541 \mathrm{~nm}$. Fluorescence lifetime images (Figure $7.5 \mathrm{~b}$ ) verified the presence of two differently organized species, being attributed to the ordered aggregates that have a long lifetime and the monomeric or defect species that have a short lifetime [17], and showed that the monomeric or defect species are mostly to be found at the boundaries of aggregated domains.

Other groups do not use chemical deposition for surface patterning, but make use of dip-pen nanolithography (DPN) techniques [18-21], which are based on atomic force microscopy (AFM) and use the AFM tip to precisely deposit fluorophores onto a chip [22, 23] or into single cells [24, 25]. One example of such a DPN technique is nanodispensing (NADIS) [26, 27]. In this NADIS technique, the inner volume of an AFM tip is milled with a Focused Ion Beam (FIB) to manufacture a dispensing channel. This channel carries the fluorophores from a reservoir on top of the AFM cantilever towards the tip of the cantilever, allowing for precise deposition of small droplets with sizes down to $70 \mathrm{~nm}$ [22].

In our collaboration with the Nanosciences group from the CEMES-CNRS institute, we imaged their NADIS patterned fluorophore droplets to study the deposition quality of sub-micron sized fluorophore (DsRed) droplets. An exemplary NADIS deposition pattern is shown in Figure 7.6a. The AFM image was provided by the Nanosciences group, and shows a 10x10 deposited array of DsRed fluorophore droplets, of which the droplets of the initial rows (top rows) grouped together. After these first rows the droplets become distinguishable, because of the

a)

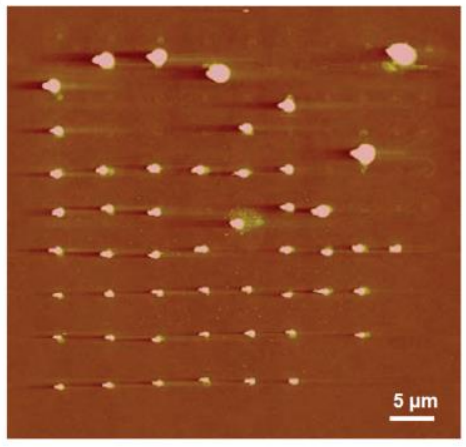

b)

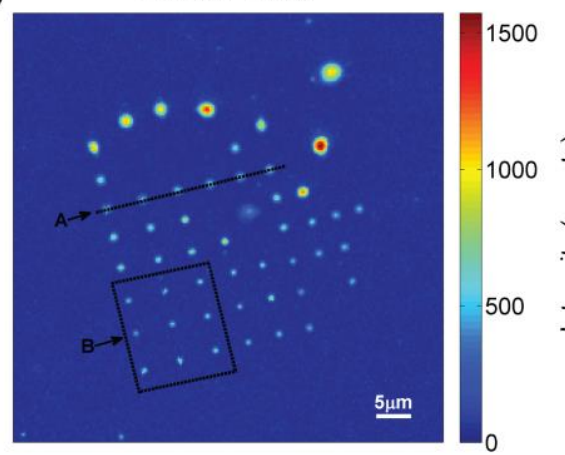

Figure 7.6 (a) AFM image of the NADIS deposited fluorophore droplets, and the corresponding fluorescence intensity image (b). 
decreased meniscus size during the pattern deposition. The droplet sizes after evaporation range from $2 \mu \mathrm{m}$ to $500 \mathrm{~nm}$.

We recorded the corresponding intensity image (Figure 7.6b), which shows well correlated features with the AFM image, verifying the presence of the fluorophores inside the deposited droplets. The intensity of the droplets scales with the droplet size, as one would expect for varying amounts of fluorophores. Furthermore, row A in Figure 7.6b shows droplet sizes of $1.1 \pm 0.1 \mu \mathrm{m}$ (FWHM), and the droplets in region B show an average size of $670 \pm 70 \mathrm{~nm}$ (FWHM). These two sizes are in agreement with the observed droplet sizes in the AFM image, when taking the convolution of the illumination point-spread-function with the droplets under consideration.

In a next step, a NADIS pattern was imaged to study the lower size limit of the droplets. The deposited pattern is shown in the AFM image in Figure 7.7a, and shows a $6 \mathrm{x} 6$ droplet array with decreasing droplet size from the upper left to the lower right corner. The intensity image (Figure $7.7 \mathrm{~b}$ ) shows a similar pattern structure as the AFM image, and shows lower intensity with decreasing droplet size. However, after the third row in the pattern, the droplets are so small that they are hardly visible in the intensity map. The low contrast of these small droplets is partly caused by the low number of fluorophores inside the droplet, but also partly due to a high level of auto-fluorescence at the substrate, which raises the background level. To improve the contrast of the small droplets, we binned the recorded fluorescence emission by accumulating emission from neighboring pixels, and then calculated the corresponding lifetime map (Figure 7.7c). The lifetime map clearly shows the presence of the smaller droplets at the bottom row of the NADIS pattern. AFM data shows that the smallest deposited droplets are $320 \mathrm{~nm}$ in size and $70 \mathrm{~nm}$ in height, giving a droplet volume of around 6 attoliter, and reflects the resolving power for

a)

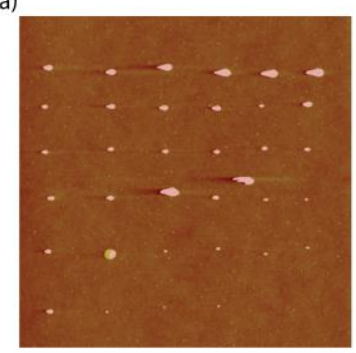

b)

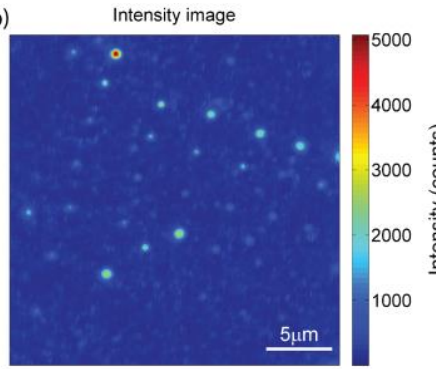

c)

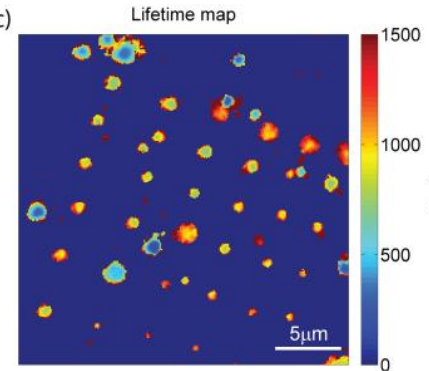

Figure 7.7 (a) AFM image of the NADIS deposited fluorophore droplets, and the corresponding fluorescence intensity image (b). Although the smallest droplets at the bottom row of the pattern are hardly visible in the intensity image, they are clearly resolvable in the fluorescence lifetime map (c). 
imaging applications of the multimodal platform.

\subsection{Concluding remarks}

In this chapter, we have shown examples that depended on the multimodality of the platform. Moreover, multimodal FLIM systems are already being used for in-vivo diagnostics [28] or are combined with non-linear harmonic generation or multiphoton microscopy [29-33] to resolve more details in biological tissue. In the future, multimodal FLIM is expected to continuously expand, for example, moving towards FRET based microscopy in living cells [34-36], and could also be combined with numerous other fluorescence-based techniques [37] to expand the modality of the system, thus providing an increasing set of observables on processes occurring at the nanoscale.

\section{REFERENCES}

1. Parker, C.A. and W.T. Rees, Correction of fluorescence spectra and measurement of fluorescence quantum efficiency. Analyst, 1960. 85(1013): p. 587-600.

2. Rurack, K., Fluorescence quantum yields: Methods of determination and standards, in Standardization and quality assurance in fluorescence measurements i, U. ReschGenger, Editor. 2008, Springer Berlin Heidelberg. p. 101-145.

3. Cesa, Y., et al., Manipulation of the local density of photonic states to elucidate fluorescent protein emission rates. Physical Chemistry Chemical Physics, 2009. 11(14): p. 2525-2531.

4. Drexhage, K.H., Influence of a dielectric interface on fluorescence decay time. Journal of Luminescence, 1970. 1-2(0): p. 693-701.

5. $\quad$ Chance, R.R., A. Prock, and R. Silbey, Lifetime of an emitting molecule near a partially reflecting surface. The Journal of Chemical Physics, 1974. 60(7): p. 27442748.

6. $\quad$ Barnes, W.L., Fluorescence near interfaces: The role of photonic mode density. Journal of Modern Optics, 1998. 45(4): p. 661-699.

7. Frimmer, M. and A.F. Koenderink, Spontaneous emission control in a tunable hybrid photonic system. Physical Review Letters, 2013. 110(21): p. 5.

8. Frimmer, M. and A.F. Koenderink, Superemitters in hybrid photonic systems: A simple lumping rule for the local density of optical states and its breakdown at the unitary limit. Physical Review B, 2012. 86(23): p. 6.

9. Chizhik, A.I., et al., Nanocavity-based determination of absolute values of photoluminescence quantum yields. Chemphyschem, 2013. 14(3): p. 505-513.

10. Nikolaev, I.S., W.L. Vos, and A.F. Koenderink, Accurate calculation of the local density of optical states in inverse-opal photonic crystals. Journal of the Optical Society of America B-Optical Physics, 2009. 26(5): p. 987-997.

11. Brokmann, X., et al., Measurement of the radiative and nonradiative decay rates of single cdse nanocrystals through a controlled modification of their spontaneous emission. Physical Review Letters, 2004. 93(10): p. 107403. 
12. Loudon, R., The quantum theory of light. 2000: Oxford university press.

13. Chizhik, A.I., I. Gregor, and J. Enderlein, Quantum yield measurement in a multicolor chromophore solution using a nanocavity. Nano Letters, 2013. 13(3): p. 1348-1351.

14. Karedla, N., et al., Absolute photoluminescence quantum yield measurement in a complex nanoscopic system with multiple overlapping states. Journal of Physical Chemistry Letters, 2014. 5(7): p. 1198-1202.

15. Preibisch, S., S. Saalfeld, and P. Tomancak, Globally optimal stitching of tiled 3d microscopic image acquisitions. Bioinformatics, 2009. 25(11): p. 1463-1465.

16. Wasserberg, D., et al., Patterning perylenes on surfaces using thiol-ene chemistry. Journal of Materials Chemistry, 2012. 22(32): p. 16606-16610.

17. Wurthner, F., et al., One-dimensional luminescent nanoaggregates of perylene bisimides. Chemical Communications, 2006(11): p. 1188-1190.

18. Jaschke, M. and H.-J. Butt, Deposition of organic material by the tip of a scanning. force microscope. Langmuir, 1995. 11(4): p. 1061-1064.

19. Salaita, K., Y. Wang, and C.A. Mirkin, Applications of dip-pen nanolithography. Nat Nano, 2007. 2(3): p. 145-155.

20. Hirtz, M., et al., Comparative height measurements of dip-pen nanolithographyproduced lipid membrane stacks with atomic force, fluorescence, and surfaceenhanced ellipsometric contrast microscopy. Langmuir, 2011. 27(18): p. 1160511608.

21. Wu, C.C., et al., Strategies for patterning biomolecules with dip-pen nanolithography. Small, 2011. 7(8): p. 989-1002.

22. Fang, A., E. Dujardin, and T. Ondarçuhu, Control of droplet size in liquid nanodispensing. Nano Letters, 2006. 6(10): p. 2368-2374.

23. Fang, A., E. Dujardin, and T. Ondarçuhu, An approach to control of droplet size in nanoscale dispensing. Journal of Physics: Conference Series, 2007. 61(1): p. 298.

24. Bitterli, J.K., Afm based single cell microinjection: Technological developements, biological experiments and biophysical analysis of probe indentation. 2012, EPFL.

25. Heinzelmann, H., et al., Nadis: A novel afm-based tool for dispensing fluids into single cells. Microscopy and Analysis-UK, 2009(134): p. 11.

26. Meister, A., et al., Nanodispenser for attoliter volume deposition using atomic force microscopy probes modified by focused-ion-beam milling. Applied Physics Letters, 2004. 85(25): p. 6260-6262.

27. Fabie, L. and T. Ondarcuhu, Writing with liquid using a nanodispenser: Spreading dynamics at the sub-micron scale. Soft Matter, 2012. 8(18): p. 4995-5001.

28. Bec, J., et al., Multispectral fluorescence lifetime imaging system for intravascular diagnostics with ultrasound guidance: In vivo validation in swine arteries. Journal of Biophotonics, 2014. 7(5): p. 281-285.

29. Sivaguru, M., et al., Imaging horse tendons using multimodal 2-photon microscopy. Methods, 2014. 66(2): p. 256-267.

30. Pelegati, V.B., et al., Harmonic optical microscopy and fluorescence lifetime imaging platform for multimodal imaging. Microscopy Research and Technique, 2012. 75(10): p. 1383-1394.

31. Adur, J., et al., Multimodal nonlinear optical microscopy used to discriminate human colon cancer. Multiphoton Microscopy in the Biomedical Sciences Xiii, 2013. 8588: p. 8. 
32. Zhao, Y.B., et al., Integrated multimodal optical microscopy for structural and functional imaging of engineered and natural skin. Journal of Biophotonics, 2012. 5(5-6): p. 437-448.

33. Mouras, R., et al., A multi-modal, multi-photon microscope for biological imaging. Multiphoton Microscopy in the Biomedical Sciences X, 2010. 7569: p. 6.

34. Raicu, V. and Deo R. Singh, Fret spectrometry: A new tool for the determination of protein quaternary structure in living cells. Biophysical Journal, 2013. 105(9): p. 1937-1945.

35. Olivo, M., C.J.H. Ho, and C.Y. Fu, Advances in fluorescence diagnosis to track footprints of cancer progression in vivo. Laser \& Photonics Reviews, 2013. 7(5): p. 646-662.

36. Sun, Y.S., et al., Forster resonance energy transfer microscopy and spectroscopy for localizing protein-protein interactions in living cells. Cytometry Part A, 2013. 83(9): p. 780-793.

37. Ishikawa-Ankerhold, H.C., R. Ankerhold, and G.P.C. Drummen, Advanced fluorescence microscopy techniques-frap, flip, flap, fret and flim. Molecules, 2012. 17(4): p. 4047-4132. 



\section{SUMMARY}

Fluorescence microscopy exploits molecular fluorescence as a simple approach to enhance the visual contrast of samples. Beyond their use in fluorescence microscopy, fluorophores have proven to be useful in a much wider range of applications, and across length-scales. For example, fluorophores have enabled probing the direct nanoenvironment of the fluorophores to study chemical processes at the nanoscale. In addition, fluorophores were also found applicable in a diverse range from solar cells and LEDs to sensing and diagnostics to quantum computing. For optimum performance, it is important to thoroughly characterize the physicochemical and photophysical properties of fluorophores prior to use. New fluorescence-based techniques like super-resolution fluorescence microscopy are rapidly developing, and are accompanied by a range of new fluorophores that push the limits of known characterization approaches. Standard ensemble characterization methods give only limited insights into the photophysics of these new fluorophores. Therefore, single molecule characterization methods are required to fully access these photophysical properties of the fluorophores. For this reason, single molecule studies have become very popular over the past three decades. At present, still not all photophysical properties can be accessed at the single emitter level. In this thesis, various techniques and methods are described that we developed to characterize single emitters, and to get detailed insights into the photophysics of quantum emitting systems.

Chapter 2 describes the assembly of the multimodal instrumentation that was built as a platform to study the photophysics of the single emitters. This multimodal fluorescence imaging instrumentation gives the flexibility to image with high sensitivity and to study multiple observables of single emitters.

We implement a modern super-continuum white-light laser in combination with an acousto-optical tunable filter (AOTF) to access for the first time the spectral absorbance properties of single emitters at room temperature. Instead of probing the absorbance directly, which is practically challenging, we access the spectral 
absorbance properties by recording excitation spectra. The development of excitation spectroscopy for single quantum dots is described in chapter 3 .

Following up on the development of single emitter excitation spectroscopy, we go one step further in chapter 4 by recording excitation spectra of single, polymer embedded, perylene dye molecules, an organic fluorophore of a class of fluorophores similar to those being used in modern fluorescence microscopy studies. In addition, we record emission spectra of the same single emitters to acquire a full spectral dataset of a single emitter. Having both the excitation and emission spectra allows one to study in full spectroscopic detail at the single molecule level phenomena such as spectral diffusion, which so far have only been studied using emission spectra. Moreover, the energy difference of the band maxima is used to systematically study the Stokes shift, a parameter that has not yet been recorded for single emitters.

In chapter 5 and chapter 6 we study another different feature of single emitters called emission intermittency, or blinking. In imaging applications the blinking of quantum dots is an ambiguous phenomenon. Although unwanted in conventional imaging, blinking is of significant relevance for super-resolution microscopy. Controlling blinking characteristics allows for optimization for both conventional and superresolution microscopy. We find indications for excitation wavelength-dependent quantum dot blinking in chapter 3. Some reports in literature support excitation wavelength-dependent blinking, while yet others contradict these observations. To investigate this disagreement we systematically study the effect of the excitation wavelength on blinking of $\mathrm{CdSe} / \mathrm{ZnS}$ core-shell quantum dots in chapter 5 , which are commonly used in imaging applications. In chapter 6 , we study the blinking mechanism of these quantum dots in detail by implementing and expanding the intensity-decay analysis technique used in chapter 5 . We identify a discrete distribution of grey states in addition to the earlier observed continuous distribution of grey states, and quantitatively measure the decay characteristics of these discrete states to learn more about the blinking mechanism. Our results lead us to propose a modification to the existing trapped charge model for quantum dot blinking to explain our observations in a manner consistent with earlier reports.

Besides using the instrumentation for single molecule studies, the high sensitivity of the platform is also advantageous for ensemble studies, allowing for fast scanning while at the same time having the possibility to analyze specific domains at the sample. Although multimodal FLIM is already being used in a wide range of 
applications (as discussed in chapter 2), we also worked on the development of new methods and applications for multimodal FLIM. In chapter 7, we describe some of these applications, giving examples of both a method and an application that require the multimodality of the platform to support their further development. 



\section{SAMENVATTING}

Fluorescentiemicroscopie is een techniek die moleculaire fluorescentie gebruikt om op een gemakkelijke manier het visuele contrast van samples te verhogen. Naast het gebruik van fluoroforen bij fluorescentiemicroscopie blijken fluoroforen ook nuttig te zijn voor een reeks aan andere toepassingen die verspreid zijn over verschillende lengteschalen. Zo hebben fluoroforen het mogelijk gemaakt om de directe nanoomgeving van fluoroforen te monitoren, zodat chemische processen kunnen worden onderzocht op de nanoschaal. Bovendien is gebleken dat fluoroforen breed toepasbaar zijn. Zo worden fluoroforen bijvoorbeeld gebruikt in zonnecellen en LEDs, maar ze kunnen ook een rol spelen bij detectie en diagnostiek en zelfs bij quantum computers. Voor een optimaal gebruik van fluoroforen is het van belang om de fysisch-chemische en fotofysische eigenschappen van fluoroforen, voorafgaand aan het gebruik, grondig te karakteriseren. Nieuwe op fluorescentie gebaseerde technieken zoals super-resolutie fluorescentiemicroscopie ontwikkelen zich razendsnel, wat gepaard gaat met de ontwikkeling van een reeks nieuwe fluoroforen, die de limitaties van bekende karakterisatietechnieken laten zien. De standaard bulk karakterisatie-technieken kunnen slechts beperkt inzicht geven in deze nieuwe fluoroforen. Om toegang te kunnen krijgen tot de volledige fotofysica van deze fluoroforen is het essentieel om naar fluorescentie van enkele moleculen te kijken. Vandaar dat enkele-molecuul studies populair zijn geworden gedurende de laatste drie decennia. Tegenwoordig zijn nog steeds niet alle meetparameters toegankelijk op het niveau van enkele fluoroforen. In dit proefschrift worden verscheidene technieken en methodes beschreven die we hebben ontwikkeld om enkele fluoroforen te karakteriseren en om gedetailleerd inzicht te krijgen in de fotofysica van quantum emitterende systemen.

Hoofstuk 2 beschrijft de assemblage van multimodale instrumentatie, die als platform dient om de fotofysica van enkele fluoroforen te karakteriseren. Deze multimodale instrumentatie biedt de flexibiteit om met hoge gevoeligheid beelden te 
verkrijgen en om verschillende fotofysische parameters van enkele fluoroforen te bestuderen.

We hebben een moderne super-continuüm wit-licht laser geïmplementeerd in combinatie met een acousto-optisch verstelbaar filter, om voor het eerst toegang te krijgen tot de spectrale absorptie-eigenschappen van enkele fluoroforen op kamertemperatuur. In plaats van absorptie te meten van een enkele fluorofoor, wat praktisch uitdagend is, kunnen we de spectrale absorptie-eigenschappen van een fluorofoor benaderen door excitatiespectra te meten. De ontwikkeling van excitatiespectroscopie voor enkele fluoroforen wordt beschreven in hoofdstuk 3 .

Aansluitend op de ontwikkeling van excitatiespectroscopie voor enkele fluoroforen gaan we in hoofdstuk 4 één stap verder, door excitatiespectra te meten van enkele peryleen kleurstof moleculen, die zijn ingebed in polymeer. Peryleen is een organische fluorofoor die deel uitmaakt van een klasse fluoroforen die regelmatig wordt gebruikt in moderne fluorescentiemicroscopie-studies. Naast excitatiespectra meten we ook emissiespectra van diezelfde enkele fluoroforen om zo de volledige spectrale dataset van een enkel fluorofoor te verkrijgen. Omdat we beschikken over zowel excitatie als emissie spectra, kunnen we in volledig spectroscopisch detail naar typische enkele-fluorofoor verschijnselen kijken zoals spectrale diffusie, wat tot nu toe alleen maar is bestudeerd met behulp van emissiespectra. Daarnaast gebruiken we het energieverschil tussen de maxima van de excitatie en emissie spectrale banden om systematisch de Stokes-verschuiving te bestuderen. Deze Stokesverschuiving is ook een parameter die tot nu toe nog niet beschikbaar was op enkelfluorofoor niveau.

In hoofdstuk 5 en hoofdstuk 6 bestuderen we een andere typische enkele-fluorofoor eigenschap genaamd 'blinking', wat neerkomt op het knipperen van de fluorofooremissie in de tijd. Blinking gedrag van halfgeleider nanocrystallen (quantum dots) is zeer onvoorspelbaar, wat zowel positive als negatieve gevolgen heeft voor fluorescentie-gebaseerde technieken. Voor de conventionele microscopie zijn de gevolgen van blinking vaak negatief omdat fluoroforen netto gezien een verlaagde quantumefficiëntie tonen en dus minder signaal en contrast opleveren in het beeld. Daarentegen is blinking juist gewenst in super-resolutie microscopie aangezien deze techniek zich volledig berust op willekeurige toestandwisselingen van fluoroforen. In hoofdstuk 3 hebben we indicaties gevonden die erop duiden dat blinking gedrag van quantum dots afhankelijk zou kunnen zijn van de golflengte van het excitatielicht. Hoewel in de literatuur een aantal onderzoeken te vinden zijn die dit fenomeen 
ondersteunen, spreken andere onderzoeken dit fenomeen weer tegen. Om deze tegenstrijdige bevindingen te onderzoeken, bestuderen we in hoofdstuk 5 systematisch het effect van de excitatiegolflengte op het blinking-gedrag van $\mathrm{CdSe} / \mathrm{ZnS}$ core-shell quantum dots. In hoofdstuk 6 bestuderen we het blinking gedrag van deze quantum dots in meer detail door gebruik te maken van de intensiteit-verval analysetechniek die ook wordt toegepast in hoofdstuk 5 . We identificeren een discrete distributie van grijze toestanden, naast de reeds geobserveerde continue distributie van grijze toestanden. We kwantificeren de vervalkarakteritieken van deze grijze toestanden om meer te weten te komen over het blinkingmechanisme. Naar aanleiding van onze resultaten stellen we voor om het huidige 'trapped-charge' model te wijzigen om onze observaties op een dusdanige manier te kunnen verklaren dat deze consistent zijn met voormalige wetenschappelijke onderzoeken.

Naast de instrumentatie te gebruiken voor enkele-fluorofoor metingen kan de hoge detectiegevoeligheid ook worden benut voor bulk studies. Hierdoor kunnen hogere scansnelheden worden behaald, terwijl de vrijheid om specifiek domeinen te karakteriseren behouden blijft. Multimodal FLIM wordt momenteel al voor een breed applicatiebereik ingezet (zoals beschreven in hoofdstuk 2). Wij hebben ons beziggehouden met de ontwikkeling van nieuwe methodes en toepassingen voor multimodal FLIM. In hoofdstuk 7 beschrijven we een aantal van deze toepassingen, waarbij we een voorbeeld geven van zowel een methode als een toepassing, die beide multimodaliteit nodig hebben voor verder ontwikkeling. 



\section{DANKWOORD}

Voor jullie ligt het proefschrift waarin de resultaten staan die ik in de afgelopen 5 jaar heb bereikt. Er staat wel één naam op de kaft, maar onderzoek doe je niet alleen. Zonder hulp van collega's, vrienden en familie was me dit nooit gelukt. Daarom wil ik hier iedereen bedanken die z'n steentje heeft bijgedragen.

First of all, I would like to thank my daily supervisor Christian. During the past years, you supported me in developing my scientific skills. Together we managed to write a number of papers on single-molecule characterization, of which I think we can be very proud of. I enjoyed our times that we spent together in Tübingen where we struggled during the day, together with Frank, Sebastien and Marcus, as a team to develop excitation spectroscopy for single emitters, and had dinner/BBQ afterwards. Thank you for all of this.

Vinod, mijn promotor, hartelijk dank dat ik jarenlang met veel plezier binnen de Nanobiophysics groep heb mogen werken aan een tal van projecten, van AFM tot single-molecule spectroscopie. We hebben naar mijn mening prettig samengewerkt om tot dit eindresultaat te komen, waar we trots op mogen zijn. Verder heb ik bewondering voor de manier waarop je met situaties omgaat waarin knopen doorhakken en timing van essentieel belang zijn; hier heb ik heel veel van geleerd.

Verder wil ik de technische staff bedanken voor alle hulp en gezelligheid die ik tijdens mijn promotie maar ook daarvoor tijdens mijn bachelor en master heb ervaren. Jullie stonden altijd direct klaar als ik hulp nodig had. In het bijzonder wil ik Kees, Robert, Aufried, Erwin, Christian en Johan bedanken, die me al die jaren hebben ondersteund met hun creativiteit, ervaren vakkennis, en niet te vergeten gezellige tijd samen.

Onze secretaresse Sylvia, jij verdient ook een pluim voor alles wat je voor mij hebt geregeld, want dat heeft mijn leven heel erg makkelijk gemaakt. Ik heb grote bewondering voor al het werk dat jij in je ééntje verzet voor de hele groep. 
Alle studenten wil ik bedanken voor hun inzet en enthousiasme. In het bijzonder wil ik Kasper bedanken voor al het werk dat je hebt verzet wat direct heeft bijgedragen aan de ontwikkeling van dit promotieonderzoek. Daarnaast heb ik erg kunnen genieten van jouw enthousiasme en gezelligheid zowel op de werkvloer als op het terras.

Ik wil mijn paranimfen Kim en Niels alvast bedanken dat zij mij zullen bijstaan tijdens de verdediging van mijn proefschrift. Maar natuurlijk vooral bedankt voor alle gezelligheid en voor de goede vriendschap. Kim, we zijn samen begonnen als bachelorstudenten in de Nanobiophysics groep en hebben nu beiden de eindstreep gehaald. Er is dan ook niets mooiers dan (net als bij jouw promotie) weer samen over deze eindstreep te gaan. Daarnaast geniet ik natuurlijk van de dagen waarop we gezellig bij elkaar op bezoek komen en onze kinderen vrolijk met elkaar kunnen zien spelen. Niels, wij hebben tijdens onze promotie veel jaren samen doorgebracht op de werkvloer en hebben nauw samengewerkt om onze singlemolecule instrumenten van scratch op te bouwen tot aan twee high-tech systemen. Daarnaast geniet ik erg van onze terrasavonden waarbij we zowel wetenschappelijke als niet-wetenschappelijke inspiratie opdoen, totdat we het laatste speciaalbiertje van de kaart hebben gehad.

I would like to thank all colleagues for their support and especially the entertaining and relaxing time that we had together during coffee breaks, lunches, BBQ's and of course during promotion parties, which gave me a lot of inspiration for my thesis.

Om dezelfde redenen wil ik graag al mijn vrienden bedanken voor alle energie die zij me hebben gegeven om tot dit eindresultaat te komen. De gezellige dagjes uit, het samen uit eten gaan, het off-road crossen en het onveilig maken van Den Haag en Hengelo (toch Tim?), hebben mij veel energie gegeven.

Mijn familie wil ik bedanken voor alle steun die ze me al die jaren, ook vóór mijn promotie, hebben gegeven. Mijn ouders Harry en Diane, bedankt dat jullie altijd voor mij klaar staan en altijd aan mij de keuze hebben gelaten om te doen wat ik leuk vind. Dit heeft er uiteindelijk voor gezorgd dat ik op de juiste plek terecht ben gekomen. Mijn twee broers Robin en Jurjen, bedankt voor jullie interesse en steun, maar vooral ook voor de ontspanning naast mijn werk. Ik bewonder jullie doorzettingsvermogen. Ik vind het erg knap dat jij, Robin, je eigen bouwbedrijf hebt opgezet en dat jij, Jurjen, na je studie Bouwkunde naar de universiteit bent gegaan 
voor een master. Bernadette, Nout en Mirthe, dank voor jullie gezelligheid en steun tijdens de ontspannen familiebijeenkomsten.

Daarnaast wil ik mijn schoonfamilie bedanken. Ans en Dick, bedankt voor alle ondersteuning en ontspanning op de camping, maar ook voor de gezellige avonden in de Ierse pub. Dat waardeer ik heel erg. Mijke, bedankt voor de leuke momenten samen op vakantie, op het terras en op de klimwand. Paul, thank you for our relaxing conversations, visits to the cinema and for providing an additional male vote in the family. Ineke en Cor, hartelijk dank voor jullie interesse en betrokkenheid. Ik kijk altijd uit naar de gezellige momenten samen.

Verder wil ik mijn naaste familieleden, Paula, Leo, Michel, Ilona, Mark, Raymond, Annemarie, Tim en Marco bedanken voor alle gezellige momenten, bijvoorbeeld op verjaardagen of familiedagen. Het verbaast me altijd weer hoe jullie in de meest bizarre en vaak grappig situaties terechtkomen. Ik geniet echt van die verhalen.

Het laatste dankwoord wil ik graag aan jullie geven, Laurien en Mayra. Laurien, jij bent voor mij heel speciaal en ik zou niet weten wat ik zonder jou moet. Je hebt al die jaren achter me gestaan, biedt altijd een luisterend oor en hebt me onvoorwaardelijk gesteund in drukke periodes. Niet alleen heb je me continu bijgestaan met het schrijven van mijn proefschrift, maar ook heb je me geholpen om alles in goede banen te laten verlopen. Ik heb veel bewondering voor jouw doorzettingsvermogen en je moet weten dat ik ontzettend trots op je ben! We hebben tussentijds een heerlijke lange vakantie naar Nieuw-Zeeland gehad, waar we nog lang met een goed gevoel op terug kunnen kijken. Hoewel we nog niet getrouwd zijn (wat dit jaar eigenlijk in de planning zat), hebben er al wel een gezinslid bij. Onze Mayra werd een aantal weken te vroeg geboren, wat overigens een flinke verschuiving in de planning veroorzaakte, en ze had al gauw de hoogste toon en het hoogste woord $:-$. Mayra, hoewel je er nog maar net achter bent gekomen hoe je een bladzijde van je houten boekje om kunt slaan en hoe je achteruit kunt inparkeren onder de kast, ben je een hele belangrijke bron van energie die me heel veel heeft gesteund tijdens mijn promotie!

\section{Martijn}





\section{CURRICULUM VITAE}

Martijn Stopel was born on the $23^{\text {rd }}$ of July 1984, in Hengelo, the Netherlands. In 2006 he obtained his Bachelor's degree Applied Physics, at the University of Twente. Subsequently, in 2008 he obtained his Master of Science degree in Applied Physics - Optics and Biophysics on the subject Atomic Force Microscopy at the biophysical engineering group at the University of Twente.

In December 2008, Martijn Stopel joined the Nanobiophysics group at the University of Twente in Enschede, The Netherlands, as $\mathrm{PhD}$ candidate under the supervision of dr. Christian Blum and Prof. dr. Vinod Subramaniam. The main research aim was to develop new methods and techniques to study the photophysics of fluorescent probes. The results of his research, involving single emitter excitation spectroscopy, single emitter Stokes shift, blinking analysis methods, and multimodal FLIM, are described in this thesis. Currently, Martijn Stopel started working as an Optical Engineer at Focal 2.0 BV, designing and engineering optical and optomechanical systems. 


\section{LIST OF PUBLICATIONS}

Blum, C., Schleifenbaum, F., Stopel, M., Peter, S., Sackrow, M., Subramaniam, V., \& Meixner, A. J. (2011). Room temperature excitation spectroscopy of single quantum dots. Beilstein Journal of Nanotechnology, 2, 516-524. doi: 10.3762/bjnano.2.56

Stopel, M. H. W., Blum, C., Subramaniam, V., Fluorescence Spectroscopy and Microscopy: Methods and Protocols, Multimodal Fluorescence Imaging Spectroscopy, Methods in Molecular Biology Volume 1076, pages 521-536, Springer Protocols 2014

Wasserberg, D., Steentjes, T., Stopel, M. H. W., Huskens, J., Blum, C., Subramaniam, V., \& Jonkheijm, P. (2012). Patterning perylenes on surfaces using thiol-ene chemistry. Journal of Materials Chemistry, 22(32), 16606-16610. doi: 10.1039/C2JM32610H

Stopel, M. H. W., Prangsma, J. C., Blum, C., \& Subramaniam, V. (2013). Blinking statistics of colloidal quantum dots at different excitation wavelengths. RSC Advances, 3, 17440-17445. doi: 10.1039/C3RA43637C

Stopel, M. H. W., Blum, C., Subramaniam, V. (2014). Excitation spectra and Stokes shift measurements of single organic dyes at room temperature, Accepted for publication in The Journal of Physical Chemistry Letters 

